\title{
A MATRIX FREE TREATMENT OF THE PROBLEM OF RIEMANN MATRICES
}

\author{
BY L. AUSLANDER AND R. TOLIMIERI ${ }^{1}$
}

In commemoration of the 75th Anniversary of the birth of A. A. Albert

Historical introduction. By the first decade of the twentieth century, the theory of the multiplier algebra of a Riemann matrix emerged as the principal part of the closely associated theories of singular correspondences between algebraic curves and algebraic correspondence on an abelian variety. A. A. Albert, in a series of papers during the 1930's, systematically characterized and classified such multiplier algebras. Although, interest shifted to more abstract problems in algebra theory, the theory of the multiplier algebra found its way into the applications of theta function theory to problems in arithmetic, most prominently evidenced by the work of C. L. Siegel in the 1950's. In more recent times, A. Weil, G. Shimura and D. Mumford, to name just a few, have found new applications for this theory. To some extent, already, in Albert's work and especially in work done after 1940, it is H. Weyl's reformulation of this theory in more representational-theoretic terms which is considered.

Our purpose in this work is two fold. Firstly, we will present the existence part of Albert's work using nilpotent algebraic group theory. The relationship between nilpotent algebraic group theory and abelian variety theory is not new to this work and goes back to the works of Cartier [10] and Weil [38] in the 1960's. Here, however, the multiplier algebra will be used to define a new collection of 2-step nilpotent algebraic groups. By 'new', we mean specifically in their relationship to the multiplier algebra. Our methods will be coordinate free and in this way the inherit identification of vector space with its dual and the accompanying matrix calculations so usually evident in this theory will be replaced by a study of those bilinear forms which lie at the heart of this theory. These bilinear forms will, in turn, be the building blocks of the nilpotent algebraic groups. The second purpose of our approach rests on the authors faith that the group theory binds in a natural fashion the various components surrounding the theory of Riemann matrices.

It may be argued that the history of the 'Riemann matrix problem' precedes Albert's work by a century. During most of this time, it must be admitted, the algebraic nature of both the problem and its eventual solution as well as its critical importance to the above-mentioned theories were not clearly perceived. The idea that the analytic and geometric problems raised

Received by the editors November 1, 1980.

1980 Mathematics Subject Classification. Primary 01A55, 01A60, 16A46, 22 E25.

${ }^{1}$ Both authors supported by NSF Grants. 
by these theories would be so served by the techniques of 'pure' algebra is a creation of the early 20th century. Conversely, the ideas and questions raised by these theories inspired certain aspects of the rapid development of this pure algebra theory. This mathematical synergism is not unique to our situation, of course. The remainder of this section will outline this history which despite the preceding discussion will be, implicitly at least, from a modern point of view (modern $\geqslant$ Albert's work). Moreover, this history will be fashioned as if Albert's work were its goal. This bizarre notion of mathematical activity, leaving by the wayside any serious discussion of theta function theory as well as any consideration of aspects of this theory tangential to our purpose, has the obvious advantage of making the line of ascent to Albert's accomplishments discernible, if not totally accurate. We apologize, in advance, for what has been omitted. Finally, we wish to record our indebtedness to the work of S. Lefschetz [18] which duly records the events up to 1928 and from which we will freely borrow and to A. Weil who set us on the right direction at the very start of this project. If we have strayed in our task, then we must absorb all the blame.

Below we have listed some of the most influential contributors to the Riemann matrix problem as well as an approximate time frame of their interest. Also, in regard to our list of references, they have been chosen with an eye towards necessity rather than sufficiency. A complete set of references before 1928 may be found in the previously-mentioned work of Lefschetz.
A. Hurwitz
$1883-1887$
G. Humbert
$1893-1901$
C. Rosati
1913-1929
G. Scorza
$1914-1925$
S. Lefschetz
$1916-1928$
A. A. Albert
$1931-1935$
H. Weyl
1934-1936

The history of the Riemann matrix begins with the study of Legendre's integral of the first kind

$$
\int \frac{d x}{\sqrt{\left(1-x^{2}\right)\left(1-k^{2} x^{2}\right)}}, \quad 0 \leqslant k^{2}<1,
$$

and with the study of complex multiplication of elliptic functions. Abel and Jacobi, working independently, observed that $\sin u$ could be defined as an inverse integral

$$
u=\int_{x=0}^{\sin u} \frac{d x}{\sqrt{1-x^{2}}}
$$

and so set out to study the properties of the function $f(u)$ defined by

$$
u=\int_{x=0}^{f(u)} \frac{d x}{\sqrt{\left(1-x^{2}\right)\left(1-k^{2} x^{2}\right)}} .
$$

This program was the starting point of the theory of Jacobi elliptic functions. It led to the development of theta function theory and is characterized by 
many beautiful functional equations whose applications to abelian variety theory and number theory form one of mathematics sparkling achievements. The basics of this latter theory can be found in Whittaker and Watson [41] and with emphasis on its applications to Riemann surface theory in Rauch and Lebowitz [21].

It is easy to see that the function $f(u)$ defined above, is well defined for real $u$. By a series of formal steps, involving the 'periodic' nature of $f(u)$ and applying Euler's addition theorem for Legendre's integral of the first kind, Abel and Jacobi extended the domain of $f(u)$ to the complex plane. The complete understanding of the power and generality of their technique, making full use of the underlying complex analysis, required a new idea. This idea was introduced and developed by B. Riemann and was his principal reason for defining the notion of a Riemann surface. Essentially it makes sense out of the problem of integrating a multi-valued function of a complex variable like

$$
\frac{1}{\sqrt{\left(1-z^{2}\right)\left(1-k^{2} z^{2}\right)}}, \quad k \text { complex }
$$

over paths in the complex plane.

Following Riemann, an algebraic curve $C$ defined by $F(z, w)=0$ can be represented by a compact Riemann surface $S$ on which the relation $F(z, w)=$ 0 defines $w=w(z)$ as a single-valued analytic function, except perhaps at a finite number of points (the branch points) where it can at worst have a pole. There are many excellent texts for the theory of Riemann surfaces and we will use the elementary parts of that theory without further reference. In particular, the complex integral, along with the standard Cauchy theory, extends unscathed to $S$. Loosely speaking, the ambiguous relationship (single-valued is nice) between $w$ and $z$ defined by the equation $F(z, w)=0$ is clarified at the expense of having to deal with the topology of $S$. Since a birational transform $C \rightarrow C^{\prime}$ of algebraic curves determines a homeomorphism $S \rightarrow S^{\prime}$ of corresponding Riemann surfaces, up to birational equivalence on $C, S$ is determined up to topological equivalence. The general theory of compact Riemann surfaces implies we may take $S$ to be a sphere with $g$ handles. This integer $g$ is a birational invariant and is called the genus of $C$ and $S$.

Denote the fundamental group of $S$ by $\pi_{1}$. As is standard, we will identify any closed oriented curve on $S$ with an element in $\pi_{1}$, relative to homotopy and call such a curve a cycle. From the model of $S$ as a sphere with $g$ handles, it follows that $\pi_{1}$ can be generated by $2 g$ cycles. The first homology group $H(S, \mathbf{Z})$ of $S$ with coefficients in $\mathbf{Z}$ can be taken as $\pi_{1} /\left\{\pi_{1}, \pi_{1}\right\} . H(S, \mathbf{Z})$ is a free abelian group on $2 g$ generators. The composition in $H(S, \mathbf{Z})$ will be written additively and any set of $2 g$ generators is called a homology basis. Special homology bases were studied by Riemann, which he called normal.

The holomorphic differentials on $S$ play the same role that analytic functions $f(z)$ or more precisely holomorphic differentials $f(z) d z$ play over domains of $\mathbf{C}$, i.e., we integrate them over curves. For a precise definition as well as proof of the next result see Rauch and Farkas [22, p. 130-132]. 
The dimension of the space of holomorphic differentials on a compact Riemann surface of genus $g$ equals $g$.

If $d v$ is a holomorphic differential on $S$ then any integral of the type $\int_{p_{0}}^{p} d v$ over some path on $S$ from $P_{0}$ to $P$ is called an abelian integral of the first kind. For a cycle $\alpha$ of $S$, the integral $\int_{\alpha} d v$ is called the period of $d v$ as to $\alpha$. It is a birational invariant of $C$. Let $\alpha_{1}, \ldots, \alpha_{2 g}$ be a homology basis for $S$ and $d v_{1}, \ldots, d v_{g}$ a basis of the homomorphic differential on $S$. Form the matrix $R=\left\{R_{j k}\right\}$ where $R_{j k}=\int_{\alpha_{k}} d v_{j}, 1 \leqslant j \leqslant g, 1 \leqslant k \leqslant 2 g$. We call $R$ a period matrix of $S$. Clearly, it depends upon the choice of bases. If $\alpha_{1}^{\prime}, \ldots, \alpha_{2 g}^{\prime}$ is another homology basis and $d v_{1}^{\prime}, \ldots, d v_{g}^{\prime}$ another basis of holomorphic differentials then the relationship between the corresponding period matrices $R^{\prime}$ and $R$ is described as follows.

$$
\alpha_{s}^{\prime}=\sum_{k} a_{s k} \alpha_{k}, \quad a_{s k} \in \mathbf{Z}
$$

Writing

$$
d v_{r}^{\prime}=\sum_{j} A_{r j} d v_{j}, \quad A_{r j} \in \mathbf{C}
$$

we have

$$
R_{r s}^{\prime}=\sum_{j, k} a_{s k} A_{r j} R_{j k}
$$

and $R^{\prime}=A R a$. In general, any two $2 g \times g$ matrices $X$ and $Y$ will be called equivalent if $X=B Y b$ where $B$ is a nonsingular $g \times g$ complex matrix and $b$ a $2 g \times 2 g$ unimodular matrix. If we simply require that $b$ be a rational nonsingular matrix then $X$ and $Y$ are called isomorphic. Thus, the period matrices of an algebraic curve determines an equivalence class of matrices.

If $R$ is a period matrix of an algebraic curve then there exists a rational alternating matrix $C$ such that

(i) $R C R^{t}=0$,

(ii) $i R C \tilde{R}^{t}$ is a positive definite Hermitian matrix.

In general, any $2 g \times g$ complex matrix satisfying these conditions is called a Riemann matrix and these conditions are known as Riemann's period relations. The matrix $C$ is called a principal matrix of $R$. When $C=\left[\begin{array}{cc}0 & 18 \\ -1 g\end{array}\right]$ these conditions were given by Riemann [23] as exactly the conditions to be satisfied by the period matrix of an algebraic curve taken with respect to a 'normal' homology basis and a 'normalized' basis of holomorphic differentials. Baker [8] was the first to explicitly write down conditions (i) and (ii) but in a different context.

The first proof that the period matrix of a compact Riemann surface is a Riemann matrix was due to Poincaré.

The Riemann matrix appears also in the theory of abelian varieties. This theory has its roots in the Riemann-Weierstrass theorem of the algebraic relation between $p+1$ periodic meromorphic functions with the same periods. The explicit concern with the underlying geometry begins with the work of Humbert $[12,13,14]$. Although, he treats primarily the case of hyperelliptic surfaces, his point of view prevails in the later work of G. Scorza, C. Rosati 
and Lefschetz. Generally speaking his techniques are applicable to the period matrices of compact Riemann surfaces having a meromorphic function with precisely two poles, counting multiplicity. For the special case of genus 2 Riemann surfaces and for the $n=2$ case of the theory we are about to discuss Humbert achieves, if one reads carefully, a complete understanding of Riemann matrices and their multiplier algebras. His techniques, depending so deeply on the low dimensionality, do not appear to generalize but in his program one sees the beginning attempts to free the theory from its geometric origins.

Suppose now that $L$ is a lattice in $\mathbf{C}^{n}$ and $\Pi=\mathbf{C}^{n} / L$ is the corresponding complex torus. Let $r_{1}, \ldots, r_{2 n}$ be an integral basis for $L$ and form the $2 n \times n$ complex matrix

$$
R=\left\{r_{1}, \ldots, r_{2 n}\right\}
$$

called the period matrix of $L$. In general, $R$ is not a Riemann matrix when $n \geqslant 2$. A meromorphic function $f(z)$ on $\mathbf{C}^{n}$ is called an abelian function for $L$ if it admits as periods all the elements of $L$, i.e., $f(z+l)=f(z), l \in L$ and $z \in \mathbf{C}$. The complex torus $\Pi$ is called an abelian variety if there exists some abelian function $f$ whose set of periods is exactly $L$. This condition has important geometric implications as to projective embeddings of $\Pi$ and analytic implications in connection with the field $\mathscr{F}(L)$ of abelian functions for $L$. Somewhat surprising perhaps is that an equivalent algebraic condition exists; namely,

$\Pi$ is an abelian variety if and only if the period matrix $R$ of $L$ is a Riemann matrix.

In this generality the result is essentially due to Scorza [32, 33]. Beginning in 1914, he initiated a comprehensive study of abstract Riemann matrices and general abelian varieties. This result however has a long history which as we have mentioned includes works of Baker and Poincaré. Its proof relies deeply on the theory of ' $n$-dimensional theta functions'.

The period matrix of an algebraic curve is always a Riemann matrix while the condition that a period matrix of a lattice be a Riemann matrix picks out exactly those complex tori having an interesting function theory, i.e., abelian variety. Since not every Riemann matrix is the period matrix of an algebraic curve, it would appear that the theory of abelian varieties is more general. We will now see that this is indeed the case. On a compact Riemann surface $S$ of genus $g$ choose a point $P_{0}$. Let $\alpha_{1}, \ldots, \alpha_{2 g}$ be a homology basis and $d v_{1}, \ldots, d v_{g}$ a basis of holomorphic differentials. Consider the corresponding period matrix $R$ and let $L$ be the lattice in $\mathbf{C}^{n}$ having $R$ as its period matrix. To each point $P$ in $S$ set

$$
j(p)=\left(\int_{p_{0}}^{p} d v_{1}, \ldots, \int_{p_{0}}^{p} d v_{g}\right)
$$

The mapping $P \rightarrow j(P)$ is not single-valued but induces a single-valued injective holomorphic mapping of $S$ into $\Pi=C^{g} / L$. The abelian variety $\Pi$ is denoted by $J(S)$ and is called the Jacobi variety of $S$. This result was formulated by Jacobi and proved by Riemann. Several authors refer to 
Riemann's work along these lines as the Jacobi inverse problem theory. Its proof, along with other related results (see [22]) is one of the important achievements of theta function theory. Since the Jacobi variety $J(S)$ has the same class of associated Riemann matrices as $S$ itself, the overlap in research involving Riemann matrices of $S$ and of arbitrary abelian varieties is great.

As yet, we have not discussed the multiplier algebra of a Riemann matrix. We shall turn to this now. Let $R$ be a $2 g \times g$ Riemann matrix. In the future we will say that $R$ has genus $g$ regardless of how it comes about. The terminology, in what follows, is by now standard; however, this was not always the case. This is by way of a warning to those who consult the literature before 1935. In fact, the changes in terminology of times betray the changes in attitude toward the theory. We will call a $2 g \times 2 g$ rational matrix $M$, not necessarily nonsingular, a multiplier of $R$ if there exists a complex $g \times g$ matrix $K$ such that $K R=R K$. We call $K$ the multiplication corresponding to $M$. It is clearly uniquely determined by $M$. In fact, the set of multipliers of $R$ and the set of multiplications of $R$ are isomorphic finite dimensional rational algebras.

In the terminology of associative algebra representation theory the multiplier algebra defines a rational representation and the algebra of multiplications defines a complex representation of one and the same abstract associative algebra. In particular, when $M=s I, s \in Q$, then $K=s I$ as well, where $I$ denotes the identity matrix. In this case we say that we have ordinary multiplication. Otherwise, we have complex multiplication. Observe we have already considered special multipliers in connection with the equivalence class of period matrices of an algebraic curve.

The study of multipliers in connection with problems arising out of the theory of algebraic correspondences of general algebraic curves was initiated by A. Hurwitz at the end of the 19th century. Beginning with [15] where he investigated the (singular) correspendences on a special algebraic curve of genus 3 occurring in the study of modular equations and in $[16,17]$ he established the importance of multipliers as concrete linear models of algebraic correspondences, thereby allowing tools from linear algebra to play a part. Although he does not appear to be greatly interested in the algebraic structure of the algebra of multipliers, he did consider sets of multipliers and the concept of linear independence of sets of multipliers. He showed, in particular, that the set of multipliers corresponding to a Riemann matrix coming from an algebraic curve of genus $g$ has dimension $\leqslant 2 g^{2}$.

Suppose $C$ is an algebraic curve of genus $g$ and $S$ its Riemann surface. An $(m, n)$-algebraic correspondence on $C$ is an algebraic transformation $T$ of $C$ into itself such that to each $z$ in $C, T$ makes correspond $n$ points $w_{1}, \ldots, w_{n}$ in $C$ and each $w$ in $C$ comes from $m$ points $z_{1}, \ldots, z_{m}$ in $C$. On $S$, we will describe the transformation $T$ by $p \rightarrow q_{1}, \ldots, q_{n}$. Choose a homology basis $\alpha_{1}, \ldots, \alpha_{2 g}$ and a basis of holomorphic differentials $d v_{1}, \ldots, d v_{g}$. As $p$ varies over $\alpha_{j}$ the corresponding points $q_{1}, \ldots, q_{n}$ describe paths whose sum we denote by $\sum_{k} a_{j k} a_{k}$. The matrix $a=\left\{a_{j k}\right\}$ describes the transformation of cycles under $T$. Also, if $f$ is a holomorphic function defined on an open subset of $S$, then the function $g$ defined by $g(p)=\Sigma_{j} f\left(g_{j}\right)$ is also holomorphic on 
some open subset of $S$. The mapping $f \rightarrow g$ induces a mapping of the holomorphic differentials, say

$$
d v_{k} \rightarrow \sum_{j} A_{j k} d v_{j}
$$

The matrix $A=\left\{A_{j k}\right\}$ describes the transformation of the holomorphic differentials and it is easy to see that $A R=R a$. Thus, $T$ determines a multiplier $a$ as well as its corresponding multiplication $A$. Those $T$ which determine complex multiplications are called singular correspondences. There is a converse to this construction depending on the theory of the Jacobi inverse problem. In fact, in terms of a notion of equivalence for algebraic transformations due to Serveri, one can establish a one-to-one correspondence between the multipliers and the equivalence classes of algebraic transformations.

The theory of complex multiplications of abelian varieties also leads to the multiplier algebra of Riemann matrices. Let $L$ be a lattice in $\mathbf{C}^{n}$ such that $\mathbf{A}=\mathbf{C}^{n} / L$ is an abelian variety. Then, the period matrix $R$ of $L$ is a Riemann matrix. The principle of complex multiplication for elliptic functions formulated by Abel can be generalized as follows. If $F$ is an abelian function for $L$ and $A$ is an $n \times n$ nonsingular complex matrix then $F\left(A_{z}^{-1}\right)$ is an abelian function for the lattice $L^{\prime}$ with period matrix $A R$. Let $\mathscr{F}(L)$ and $\mathscr{F}\left(L^{\prime}\right)$ be the fields of abelian functions for $L$ and $L^{\prime}$, respectively. A necessary and sufficient condition that the elements of $\mathscr{F}(L)$ depend algebraically on those of $\mathscr{F}\left(L^{\prime}\right)$ and vice versa is that $L$ and $L^{\prime}$ be commensurable, i.e., $L \cap L^{\prime}$ is of finite index in both $L$ and $L^{\prime}$. In terms of the period matrices, this is equivalent to the existence of a $2 n \times 2 n$ rational nonsingular matrix $a$ such that $A R=R a$. Thus, if we drop the conditions of nonsingularity for $A$ and $a$ we come to the definition of multiplier and multiplication previously given for $R$.

The properties of a Riemann matrix $R$ are of course closely related to the properties of its multiplier algebra. Almost simultaneously, from 1914 onwards, Scorza [35] in connection with complex multiplications of abelian varieties and Rosati $[27,29,30]$ in connection with singular correspondences of algebraic curves, studied the theory of Riemann matrices and their multiplier algebras. As we have indicated, Humbert had previously studied this theory for Riemann matrices of genus 2. Somewhat later Lefschetz summarized in [18] Scorza's and Rosati's results as well as providing a history of this theory up to that time. Also, although his interests were more in line with applications to geometry and analysis, in [19], a work for which he won the Bordin prize in 1919, he provided the most complete classification theory of Riemann matrices and their multiplier algebras up to that time. His interests, as the title would imply, were the study of certain numerical invariants connected to Riemann matrices. We will now outline in greater detail the ideas prominent during this period from 1915 to 1930 . This will be important if we want to clearly understand what changes Albert brought to the theory.

Scorza [33] was the first to study the properties of abstract Riemann matrices. Along with Rosati, he studied certain numerical invariants which 
provided information about the space of multipliers. Equally important, he continued the study of the problem of reducing a Riemann matrix $R$ into its 'pure' components. Poincare was the first to see the importance of this problem and in fact proved the main results when the Riemann matrix comes from an algebraic curve.

Also, although linear algebra techniques were coming into their own, Scorza relied on projective geometric techniques for many of his early results. A decade later Albert gave a simpler proof. A Riemann matrix $R$ is called impure if it can be written

$$
R=\left[\begin{array}{cc}
R_{1} & 0 \\
0 & R_{2}
\end{array}\right]
$$

where $R_{1}$ and $R_{2}$ are Riemann matrices of genus less than the genus of $g$ of $R$. The decomposition theorem is as follows.

Every Riemann matrix $R$ can be written

$$
R=\left[\begin{array}{ccc}
R_{1} & & 0 \\
& \ddots & \\
0 & & R_{p}
\end{array}\right]
$$

where all the $R_{j}$ are pure, i.e., not impure and any two are either identical or nonisomorphic.

The importance of this result to the study of the multiplier algebra of $R$ is that we may reduce the problem to the study of pure Riemann matrices and in this case the associated multiplier algebra is a division algebra. We will have more to say about this result in the next section.

Scorza [34] and Lefschetz [19] studied the important problem of classifying a Riemann matrix of a given genus according to the existence of certain multiplications. The linear algebra and elementary field theory techniques introduced by Scorza and fully exploited by Lefschetz would eventually betray the algebraic nature of the classification problem. Later we will discuss Rosati's fundamental contribution as well, which although known at this time belongs truly to another period.

Consider a Riemann matrix $R$ of genus $g$ and suppose $a$ is a multiplier of $R$ and $A$ is its corresponding multiplication. Thus, $A R=R a$. Let $M$ be the multiplier algebra of $R$. Since $A$ and $a$ are matrices we may consider their respective characteristic equations:

$$
\phi(s)=\operatorname{det}(A-x I)=0 \text { and } F(x)=\operatorname{det}(a-x I)=0 .
$$

Many of the linear algebra techniques which are by now so well known were at the time of Lefschetz only several decades old and their application to the Riemann matrix theory, already implicit in the work of Humbert, was an important contribution of firstly, Scorza and later, Rosati and Lefschetz.

It is standard that $F(a)=0$ (Cayley-Hamilton), and so if $f$ is the minimal polynomial of $a$ then $f$ divides $F$ and they have the same roots. Suppose that $R$ is pure, for the moment. Then $f$ is always irreducible, and $F=f^{r}$, for some 
integer $r$. Since the degree of $F$ is $2 g$, the degree of $f$, say $q$, must divide $2 g$, i.e., $q r=2 g$. If $h$ is the dimension of the multiplier algebra, then we must also have, by purely linear algebra techniques, that $q$ divides $h$. The history of the integer $h$, as an invariant of $R$, is interesting and was introduced into the theory in a less geometric fashion. For a Riemann matrix $R$, the dimension of the space of all rational $2 g \times 2 g$ matrices $C$ satisfying $R C R^{t}=0$ was called by Scorza [32] the index of multiplication of $R$. This index of multiplication was just one of the several discussed by Scorza. In an early fundamental result, he proved that $h$ was equal to the index of multiplication of $R$.

Lefschetz [19] made a detailed study of the following problem.

If $f$ is an irreducible polynomial and $r$ is an integer then classify those Riemann matrices $R$ which admit a multiplier whose characteristic polynomial is $f^{r}(x)=0$, for some integer $r$.

In particular he studied the case when $f$ had all real roots and the case when $f$ had totally complex roots. This continued a program of study initially considered by Scorza. Later we will see that these results inspired Albert as well. Although we will not discuss Lefschetz's classification theory in any detail, we should point out that this achievement was a crown jewel in the theory of 'applied' linear algebra and field theory, especially Galois theory. Its influence on Albert's work cannot be overestimated. It predestined the nature of Albert's results and in doing so exerted an important influence on the theory of associative algebra.

The theory of associative algebra had made substantial progress during the early part of the 1900's. Its techniques founded by J. H. M. Wedderburn and developed by Albert, R. Brauer, H. Hasse and E. Noether, to name just a few, would finally bring about a solution of the multiplier algebra classification problem. From what we have written, it would almost appear as if mathematical theories rise and fall as Gibbon's Rome. We have an immediate contradiction. The first topic of associative algebra theory, which we consider is due to Rosati [28, 31], was known to Lefschetz (bottom of p. 383 of [18]) and constituted an important tool in the theory of singular correspondences. We will introduce it as Albert considered it (see [4]). Suppose $R$ is a pure Riemann matrix. Then the multiplier algebra $D$ of $R$ is a rational division algebra of finite dimension. The center $k$ must therefore be a number field. The dimension of $D$ over its center $k$ must be of the form $n^{2}, n$ an integer, and we call $n$ the degree of $D$. Thus $D$ is an example of anormal rational division algebra. It also admits a positive involution. For if $C$ is a principal matrix for $R$, then the mapping $X \rightarrow C^{-1} X^{t} C$, determines a positive involution of $D$ and is called a Rosati involution. Denote it by $\gamma$. Albert [1] made a complete study of normal rational division algebras admitting a positive involution. The following results, however, are due to Rosati [24, 25]. An element $x$ in $D$ is called $\gamma$-symmetric if $\gamma(x)=x$ and $\gamma$-skew-symmetric if $\gamma(x)=-x$. Rosati showed that if $x$ is symmetric (with respect to any Rosati involution) then the roots of its minimal equation are all real while if $x$ is skew-symmetric these roots are purely imaginary. Albert [2] gave simpler algebraic proofs of these results and proved converses as well.

We come now to Albert's work. Consider, as above, the multiplier algebra 
$D$ of a pure Riemann matrix $R$ and let $k$ be its center. Using a Rosati involution, Albert [2] was able to distinguish two separate classes of multiplier algebras. Firstly, $\gamma$ defines an automorphism of $k$. If $\gamma$ acts by the identity mapping on $k$ we say $D$ is of the first kind while, otherwise, we say $D$ is of the second kind. These definitions are independent of which Rosati involution is considered. For $D$ of the first kind, the center $k$ must be a totally real number field. If $D$ is of the second kind, then $k$ is a quadratic totally imaginary extension of a totally real field $k_{0}$ and we may write $k=k_{0}(x)$ where $\gamma(x)=-x$. As innocent as this result appears it is a 'global' result and for this reason is crucial in understanding Albert's philosphy.

Albert [3] showed that if $D$ was of this first kind then it is either a totally real field or a generalized quaternion algebra over a totally real field. It is important to understand that Albert is now working at building a theory within abstract associative algebra theory, although its application to the Riemann matrix problem is never far removed. Suppose then, for the moment, we consider $D$ as any normal division algebra over a number field $k$ of degree $n$. Using the important invariant of the exponent (see [1, p. 76]), Albert proved the next result (in a slightly different language).

If $D$ admits an involution of the first kind, then $D$ has degree one or two over its center.

This result appears weaker than Albert's main result in [3] but, in fact, they are (not trivially) equivalent and at any rate sufficient for our purposes (see [1, Chapter 10]).

The next natural problem to consider is which generalized quaternion algebras over totally real fields are in fact the multiplier algebra of Riemann matrices of the first kind. Albert does more, as we shall see, and studies how the degree of the center of such an algebra is related to the genus of the Riemann matrix.

In [2], Albert refined a result of Scorza in connection with the relationship between the multiplicity index $h$ and the genus $g$ of a Riemann matrix. Scorza had showed that $h \leqslant 2 g$ while Albert proved $h$ divides $2 g$. This restriction between the numerical invariants of a Riemann matrix provided sufficient capital, in light of the preceding result, to completely classify and construct the multiplier algebras of the first kind. These results are found in $[4,5]$. We list these results essentially as Albert first stated them.

(I) Let $k$ be a totally real number field of degree $t$ over $Q$. Then there exists a Riemann matrix of genus $g$ having $k$ as its multiplier algebra if and only if $t$ divides $g$.

(II) Let $D=\mathbf{K}(a, b)$ be a generalized quaternion algebra over a totally real field of degree $t$ over $Q$ (see $\S 6$ of this work for the notation). Then, if $a$ and $b$ are totally positive, there exists Riemann matrix of genus $g$ with $D$ as its multiplier algebra if and only if $2 t$ divides $g$.

(III) Let $D=\mathbf{K}(a, b)$, where $a$ and $b$ are totally negative. Then, there exists a Riemann matrix of genus $g$ having $D$ as its multiplier algebra if and only if $g \neq 2 t$ and $2 t$ divides $g$.

(IV) The above list, (I)-(III), exhausts all the multiplier algebras of the first kind. 
In fact, Albert considered more general versions of these results and dealt with Riemann matrices over number fields.

The case of multiplier algebras of the second kind proved to be more difficult to handle. It required a greater input of associative algebra theory (some yet to be built). The following discussion will necessarily be brief. In retrospect, there were two ideas missing in the early 1930's. First there was needed information about the relationship between rational division algebras admitting involutions and cyclic algebras. The class of cyclic algebras was introduced by Dickson (1906). Their definitions may be found in the main body of this work. The general theory of cyclic algebras had been built before Albert's work by Brauer, Hasse [11], and Wedderburn [37]. The important result is found in Brauer, Hasse and Noether [9] which implies that every rational division algebra is a cyclic algebra over its center. If $D$ is a rational division algebra of degree $n$ over its center $k$, then a cyclic extension of degree $n, K$ of $k$, can be chosen in $D$ which 'splits' $D$ over $k$, in a manner made precise in $\$ 7$. Albert studied rational division algebras admitting positive involutions of the second kind and showed how this splitting field $K$ could be specially chosen with respect to the involution. The complete theory can be found in [1]. The second topic which would eventually play a role was the notion of crossed products and the related idea of factor sets $[1,40]$. The latter had already found its way into the general theory.

In [6], Albert went directly to the problem at hand. In terms of cyclic algebras and positive involutions, he defined a new type of algebra by a set of conditions which he numbered (19)-(29). We will denote these conditions by *. Then he proved the following result.

The multiplication algebra of any pure Riemann matrix of the second kind is an algebra satisfying * and any division algebra satisfying $*$ is the multiplication algebra of some pure Riemann matrix of the second kind.

Observe that this result is not as specific as those given in (I)-(III) since no mention is made of restrictions upon the relationship between genus and degree. Additional information can be found in Siegel [36]. Loosely summarizing Albert's work, we may say he studied division algebra over $Q$ with positive involution, characterized them, and showed that except for certain exceptions they could be realized as the multiplier algebras of some Riemann matrix.

The final works we consider are due to Weyl [39, 40]. Although they come after Albert's original solution, they determined subsequent rethinking and reformulation of Albert's work, by Albert himself [7], by Siegel [36] and in much of what could be called the current point of view. It will certainly be critical for us. Generally speaking the philosophy behind Weyl's reformulation is transparent. Consider again the development of Riemann matrices from the point of view of abelian varieties. There one begins with a complex vector space $\mathbf{C}^{n}$ and studies lattices $L$ of $\mathbf{C}^{n}$. The period matrices $R$ of these lattices may or may not satisfy the Riemann period relations but when such an $R$ does, then the complex torus $\mathbf{C}^{n} / L$ is an abelian variety and we study 
its multiplier algebra. In Weyl's approach the original situation is reversed. Now a lattice $L$, usually $\mathbf{Z}^{2 n}$, is specified in $\mathbf{R}^{2 n}$ and one considers complex structures $J$ on $\mathbf{R}^{2 n}$ and the resulting complex torus $\Pi=\left(\mathbf{R}^{2 n} / L, J\right)$. For more details on these concepts see $\$ 2$ of this work. Loosely speaking $J$ plays the role of $R$ and has the advantage of being a real matrix. The multiplier algebra of $J$ is defined as the collection of all $2 n \times 2 n$ rational matrices $\left(L=\mathbf{Z}^{2 n}\right)$ which commute with $J$. Thus the unsymmetric condition for a multiplier in the pre-Weyl theory gives way to a symmetric condition. We will now describe the Weyl reformulation.

Suppose $R$ is a Riemann matrix and $C$ is a principal matrix for $R$. Let $\Omega=\left[\frac{R}{R}\right]$. The defining conditions for $C$ can be rewritten as

$$
i \Omega C \bar{\Omega}^{+}=\left[\begin{array}{cc}
H & 0 \\
0 & -\bar{H}
\end{array}\right]
$$

where $H$ is a positive definite Hermitian matrix. It follows that $\Omega$ is nonsingular. Let

$$
J=\Omega^{-1}\left[\begin{array}{cc}
-i E n & 0 \\
0 & i E n
\end{array}\right]
$$

where $E_{n}$ is the $n \times n$ identity matrix. Then Weyl proves the following result.

(i) $J$ is a real $2 n \times 2 n$ matrix,

(ii) $J^{2}=-E_{2 n}$,

(iii) $C J$ is a positive definite symmetric matrix.

Conversely, if $J^{\prime}$ is any matrix satisfying these conditions for some rational alternating $2 n \times 2 n$ matrix $C^{\prime}$ then $J^{\prime}$ comes from a Riemann matrix $R^{\prime}$ which has $C^{\prime}$ as a principal matrix. Moreover, $R^{\prime}$ is uniquely determined up to left multiplication by a complex $n \times n$ matrix.

REMARKs. Conditions (i) and (ii) above define $J$ as a complex structure on $\mathbf{R}^{2 n}$. If $J$ satisfies (i) and (ii) then the existence of a rational alternating $2 n \times 2 n$ matrix $C$ is exactly what is needed to make $\left(\mathbf{R}^{2 n} / \mathbf{Z}^{2 n}, J\right)$ into an abelian variety.

Finally, in the notation leading up to the theorem, the multiplier algebra $M$ of $R$ is the same as the multiplier algebra of $J$.

\section{REFERENCES TO HISTORICAL INTRODUCTION}

\section{A. A. Albert}

1. Structures of algebras, Amer. Math. Soc. Colloq. Publ., vol. 24, Amer. Math. Soc., Providence, R. I., 1939.

2. On the structure of pure Riemann matrices with non-commutative multiplication algebras, Palermo Rendiconti 55 (1931), 57-115.

3. Algebras of degree $2^{e}$ and pure Riemann matrices, Ann. of Math. (2) 33 (1932), 311-318.

4. On the construction of Riemann matrices. I, Ann. of Math. (2) 35 (1934), 1-18.

5. On the construction of Riemann matrices. II, Ann. of Math. (2) 36 (1935), 376-394.

6. A solution of the principal problem in the theory of Riemann matrices, Ann. of Math. (2) 35 (1934) 500-515.

7. Involutorial simple algebras and real Riemann matrices, Ann. of Math. (2) 36 (1935), 886-964.

H. F. BAKER

8. An introduction to the theory of multiply periodic functions, Cambridge, 1 (1907). 
R. BRAuer, H. HASSe, E. Noether

9. Beweis eines Heuptsatzes in der Theorie der Algebren, Crellés J. 167 (1931), 399-404.

P. CARTIER

10. Quantum mechanical commutative relations and theta functions, Proc. Sympos. Pure Math., vol. 9, Amer. Math. Soc., Providence, R. I., 1965, pp. 361-387.

H. HASSE

11. Theory of cyclic algebra over an algebraic number field, Trans. Amer. Math. Soc. 34 (1932), 170-214.

G. Humbert

12. Sur les fonction abéliennes singulières, J. de Math. (5) 5 (1899), 233-350.

13. Sur les fonction abéliennes singulières, J. de Math. (5) 6 (1900), 279-386.

14. Sur les fonction abéliennes singulières, J. de Math. 7 (1901), 97-123.

A. HuRwitz

15. Zur Theorie der Modulargleichungen, Gott. Nach. (1883), 350-363.

16. Über Algebraische Korrespondensen und das Verallegemeinerte Korrespondenzprinzip, Leipz. Ab. (1886), 10-38.

17. Über Algebraische Korresponenzen und das Verallgemeinerte Korrespondenzprinzip, Math. Ann 28 (1887), 561-585.

\section{S. LEFSCHETZ}

18. Selected topics in algebraic geometry, Bull. of N.B.C., No. 63, Washington, 1928 (reprinted, Chelsea, 1970).

19. On certain numerical invariants of algebraic varieties with applications to abelian varieties, Trans. Amer. Math. Soc. 22 (1921) 327-482; translation of the memoir awarded the Bordin Prize by the Paris Academy of Sciences for the year 1919.

H. POINCARÉ

20. Sur la réduction des intégrales Abéliennes, Oeuvres t. III (1934), 333-351.

H. RaUCh AND A. LeIBOwitz

21. Elliptic functions, theta functions and Riemann surfaces, Williams \& Wilkins Co., Baltimore, Maryland, 1973.

H. RAUCH AND H. FARKAS

22. Theta functions with applications to Riemann surfaces, Williams \& Wilkins Co., Baltimore, Maryland, 1974.

B. RIEMANN

23. Theorie der abelschen Funktionen, Gesamm. Math. Werke, Dover, New York, 1953, pp. 84-142.

\section{Rosati}

24. Sulle corrispondenze plurivalenti fra i punti di una curva algebrica, Atti Accad. Sci. Torino 51 (1915-16), 991-1014.

25. Sulle valenze delle corrispondenze algebriche fra $i$ punti di una curva algebrica, ibid. 53 (1917-18), 5-22.

26. Intorno alle corrispondenze simmetriche singolari sopra una curva di genere 2, Rend. Circ. Mat. Palermo 44 (1920), 307-335.

27. Sulle corrispondenze fra $i$ punti di una curva algebrica e, in particolare, fra $i$ punti di una curva di genere due, Ann. Mat. Pura Appl. (3) 25 (1916), 1-32.

28. Sulle corrispondenze algebriche fra i punti di due curve algebriche, ibid. (3) 28 (1918), 35-60.

29. Sulle corrispondenze algebriche fra i punti di una curva algebrica, Atti Accad. Lincei Rend. Cl. Sci. Fis. Mat. Nat. (5) 22 (1913), $2^{\circ}$ Semestre, 385-390, 431-436.

30. Sulle corrispondenze fra $i$ punti di una curva algebrica $e$, in particolare, fra $i$ punti di una curva di genere due, ibid. (5) 24 (1915), $2^{\circ}$ Semestre, 182-184.

31. Sulle matrici di Riemann, Rend Circ. Mat. Palermo 53 (1929), 79-134. 


\section{G. SCORZA}

32. Sopra alcune notevoli madrici riemanniane, Torino Atti. 53 (1917), 1008-1017.

33. Intorno alla teoria generale della matrici di Riemann, Rend. Circ. Mat. Palermo 41 (1916), 263-379.

34. Le algebre di ordine qualunque e le matrici di Riemann, Rend. Circ. Mat. Palermo, 45 (1921), 1-204.

35. Papers from 1914-1916, Rom. Acc. L. Rend.

C. L. SIEGEL

36. Lectures on Riemann matrices, Tata Institute of Fundamental Research, Bombay, 1963.

J. H. M. WEDDERBURN

37. On division algebras, Trans. Amer. Math. Soc. 22 (1921), 129-135.

A. WeIL

38. Sur certaine groupes d'operateurs unitaires, Acta. Math. 111 (1964), 143-211.

H. WEYL

39. On generalized Riemann matrices, Ann. of Math. (2) 35 (1934), 714-729.

40. Generalized Riemann matrices and factor sets, Ann. of Math. (2) 37 (1936), 704-745.

E. T. WhitTAKer AND G. N. WATSON

41. A course of modern analysis, Cambridge, 1902.

More recent discussions and applications of the multiplier algebra can be found in the following works:

I. C. L. Siegel, Advanced topics in analytic number theory, Tata Institute of Fundamental Research, Bombay, India, 1961.

Gottingen, 1951.

Die Modulgruppe in einer einfachen involutorischen algebra, Festschrift Akad. Miss.

Along with the above referenced 36 , these works study the theory in connection to certain modular groups (Hilbert modular group).

II. A. Weil, Algebras with involutions and the classical groups, J. Indian. Math. Soc. 24 (1960), $589-623$.

III. G. Shimura, Introduction to the arithmetic theory of automorphic functions, Kanô Memorial Lectures No. 1 Iwanami Shoten, Publishers and Princeton University Press, 1971.

III. G. Shimura and Y. Taniyama, Complex multiplications of Abelian varieties and its applications to number theory, Publ. Math. Soc. Japan no. 6, 1961.

The first work is a source for many related topics while the second deals mainly with arithmetic applications. In particular, multiplier algebras are considered and constructed which contain quadratic imaginary extensions of totally real fields, i.e. $C M$ fields.

IV. D. Mumford, Curves and their Jacobians, University of Michigan Press, Ann Arbor, 1976.

A complete history of all of Albert's works may be found in:

V. N. Jacobson, Abraham Adrian Albert 1905-1972, Bull. Amer. Math. Soc. 80 (1974), 1075-1100.

1. Algebraic preliminaries. We will consider solely fields $K$ of characteristic 0 , namely the rational field $Q$, the real field $\mathbf{R}$, the complex field $\mathbf{C}$ or an algebraic number field $\mathfrak{f}$. All vector spaces and algebras over $K$ will be finite dimensional.

A. Weil in his Acta paper [6], Sur certaines groupes d'operateurs unitaires, firmly established the relationship between abelian variety theory and nilpotent group theory. This work has been continued in several directions and by 
this time it is apparent that these two theories are closely interwoven. For a discussion of these ideas most suitable for this paper see [2]. In this work we will discuss what the authors believe to be a basic connection between these theories, involving on the one hand the multiplication algebra of an abelian variety and on the other certain nilpotent groups and homomorphism built from these multiplication algebras. In the next section we will recall some of the theory of abelian varieties and their multiplication algebras as well as describe the problems and methods of this work. Here, we will assemble some general results on the nilpotent groups which occur in abelian variety theory.

Let $\mathfrak{A}$ be an associative algebra over $K$ of the form

$$
\mathfrak{U}=K \cdot 1 \oplus \Re
$$

where 1 is the identity of $\mathfrak{U}$ and $\mathscr{R}$ is its radical. Consider the subset $N=N(\Re)$ of $\mathfrak{A}$ given by

$$
N=\{1+\alpha: \alpha \in \Re\} .
$$

$N$ is a subgroup of the multiplicative group of units $\mathfrak{U}^{x}$ of $\mathfrak{A}$ which may be seen as follows. The product of $1+\alpha, 1+\beta$ in $N$ is given by $(1+\alpha)(1+\beta)$ $=1+(\alpha+\beta+\alpha \beta)$ which is again in $N$ and if $\Re^{k+1}=(0)$ then the multiplicative inverse of $1+\alpha$ in $N$ is given by $(1+\alpha)^{-1}=1-\alpha$ $+\cdots+(-1)^{k} \alpha^{k}$ which is also in $N$.

For any ideal $q$ of $R$ we set

$$
N(\mathscr{G})=\{1+\alpha: \alpha \in \mathscr{G}\}
$$

and arguing as above $N(\mathscr{G})$ is a normal subgroup of $N=N(\Re)$. Also, it is easy to see that

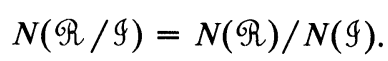

The group $N=N(\Re)$ has a faithful representation $\rho$ in $G L(\mathscr{U})$, the nonsingular $K$-linear endomorphisms of $\mathfrak{A}$ defined by setting

$$
\rho(1+\alpha)(\beta)=(1+\alpha) \beta
$$

where $1+\alpha \in N$ and $\beta \in \mathfrak{A}$. Since $\mathscr{R}$ is solvable, a basis for $\mathfrak{A}$ over $K$ may be chosen so that the corresponding matrix representation, also denoted by $\rho$, satisfies $\rho(N) \subset U(m, K)$ where $m=\operatorname{dim}_{K} \mathfrak{U}$ and $U(m, K)$ is the group of upper triangular unipotent matrices. In particular, $N$ must be nilpotent. In fact, $N$ is a $K$-nilpotent algebraic group in the sense of the following definition.

Definition. A group $G$ is called a $K$-nilpotent algebraic group if there is a faithful matrix representation

$$
q: G \rightarrow U\left(K, m^{\prime}\right), \quad m^{\prime} \in Z_{+}^{x},
$$

such that $q(G)$ is a $K$-algebraic variety in $U\left(K, m^{\prime}\right)$.

The product rule for $N$ considered above shows that $\rho(N)$ is the set of zeroes in $U(K, m)$ of a system of linear equations over $K$.

We shall be especially interested in 2-step nilpotent groups $G$, namely, groups where $[G, G]$ is central. If $G$ is a 2-step $K$-nilpotent algebraic group it 
is easy to see that $G$ satisfies an exact sequence

$$
0 \rightarrow V_{1} \rightarrow G \rightarrow V_{2} \rightarrow 0
$$

where $V_{1}, V_{2}$ are vector spaces over $K$.

We will now apply the above construction to two especially relevant examples.

EXAMPLE. 1. Let $V$ be a vector space over $K$ and consider $\mathfrak{A}=\Lambda(V)$, the exterior algebra of $V$. Then

$$
\Lambda(V)=K \cdot 1 \oplus \Re
$$

where $R=\sum_{i>0} \Lambda^{i}(V)$ is the radical of $\Lambda(V)$. Hence, we may form the $K$-nilpotent algebraic group $N(\Lambda(V))$.

EXAMPLE 2. Let $\mathscr{G}=\sum_{i>2} \Lambda^{i}(V) \subset \Re$, the notation being as in Example 1.Then 9 is an ideal of $\Lambda(V)$ and we may form

$$
\mathscr{F}_{2}(V)=N(\Re / \mathscr{Q}) \text {. }
$$

The group $\mathscr{F}_{2}(V)$ will be extremely important for this work. An explicit description of $\mathscr{F}_{2}(V)$ can be given as follows. As a set $\mathscr{F}_{2}(V)=V \times(V \wedge V)$ and the group law of composition is given by

$$
\left(v_{1}, w_{1}\right)\left(v_{2}, w_{2}\right)=\left(v_{1}+v_{2}, w_{1}+w_{2}+v_{1} \wedge v_{2}\right)
$$

where $v_{1}, v_{2} \in V$ and $w_{1}, w_{2} \in V \wedge V$.

$\mathscr{F}_{2}(V)$ is a 2-step nilpotent algebraic group with center $(0, w), w \in V \wedge V$, and is called the free 2-step $K$-nilpotent group of $V$. The reason for the name 'free' is the following. We will identify $V$ with

$$
\mathscr{F}_{2}(V) /\left[\mathscr{F}_{2}(V), \mathscr{F}_{2}(V)\right]
$$

in the obvious way. If $G$ is a 2-step $K$-nilpotent algebraic group and

$$
F: V \rightarrow G /[G, G]
$$

is $K$-linear then there exists a homomorphism $f: \mathscr{F}_{2}(V) \rightarrow G$ whose kernel is a $K$-algebraic group and which makes the following diagram commute:

$$
\begin{gathered}
\mathscr{F}_{2}(V) \stackrel{F}{\rightarrow} G \\
\swarrow \\
\stackrel{f}{\rightarrow} G /[G, G]
\end{gathered}
$$

In particular, every $K$-linear mapping $X$ of $V$ determines a unique homomorphism, also denoted by $X$, of $\mathscr{F}_{2}(V)$.

There are certain homomorphisms of $\mathscr{F}_{2}(V)$ that will play an essential role in our theory. We will digress to introduce some necessary notation. Let $V$ and $W$ be vector spaces over $K$. We use $\operatorname{Hom}(V, W)$ to denote the space of $K$-linear maps from $V$ into $W$ and, in particular, let $V^{*}=\operatorname{Hom}(V, K)$, the dual of $V$. We will implicitly identity $V$ and $V^{*}$. For $T \in \operatorname{Hom}(V, W)$ we let $T^{*} \in \operatorname{Hom}\left(W^{*}, V^{*}\right)$ be the dual of the transformation $T$ defined by $T^{*}(F)(v)$ $=F(T(v)), v \in V$ and $F \in W^{*}$. 
Let $\operatorname{Bil}(V)$ denote the space of bilinear forms $B$ of $V, B: V \times V \rightarrow K$. We will identify $\operatorname{Bil}(V)$ with $\operatorname{Hom}\left(V, V^{*}\right)$ as follows. For $B \in \operatorname{Bil}(V)$ put $L(B) \in$ $\operatorname{Hom}\left(V, V^{*}\right)$ where $L(B)(v)(w)=B(v, w), v, w \in V$. Since $L(B) \in$ $\operatorname{Hom}\left(V, V^{*}\right)$ we have $L(B)^{*} \in \operatorname{Hom}\left(V, V^{*}\right)$ under the canonical identification of $V$ and $V^{* *}$. We say that $B$ is alternating if $L(B)^{*}=-L(B)$ and symmetric if $L(B)^{*}=L(B)$. These definitions are identical with the usual matrix definitions. In the same way, we say $B$ is nonsingular if $L(B)$ is nonsingular. The space of alternating bilinear forms will be denoted by $\operatorname{Alt}(V)$ and the space of symmetric bilinear forms will be denoted by $\operatorname{Sym}(V)$. Then, clearly,

$$
\operatorname{Bil}(V)=\operatorname{Alt}(V) \oplus \operatorname{Sym}(V) .
$$

The nonsingular bilinear forms are denoted by $\operatorname{Bil}^{x}(V)$ and $\operatorname{Alt}^{x}(V)=\operatorname{Alt}(V)$ $\cap \operatorname{Bil}^{x}(V), \operatorname{Sym}^{x}(V)=\operatorname{Sym}(V) \cap \operatorname{Bil}^{x}(V)$.

Let $\mathscr{F}_{2}(V)$ again denote the free 2-step $K$-nilpotent group over $V$. Its center is $V \wedge V$. The dual of $V \wedge V$ can be identified with $\operatorname{Alt}(V)$. For $A \in \operatorname{Alt}(V)$ we let $l(A) \in(V \wedge V)^{*}$ denote the unique element making the following diagram commute:

$$
\begin{aligned}
& V \times V \stackrel{\wedge}{\rightarrow} V \wedge V \\
& \downarrow A A \quad \swarrow l(A) \\
& K
\end{aligned}
$$

Now for $A \in \operatorname{Alt}(V)$ we may define a group structure $N(A)$ on the set $V \times K$ whose law of multiplication is

$$
\left(v_{1}, \alpha_{1}\right)\left(v_{2}, \alpha_{2}\right)=\left(v_{1}+v_{2}, \alpha_{1}+\alpha_{2}+A\left(v_{1}, v_{2}\right)\right)
$$

where $v_{1}, v_{2} \in V$ and $\alpha_{1}, \alpha_{2} \in K$. Then $N(A)$ is a 2-step $K$-nilpotent algebraic group whose center contains $\{(0, \alpha): \alpha \in K\}$ and $N(A)$ modulo its center is abelian. Moreover, we have the surjective homomorphism $P=P_{A}$,

$$
P: \mathscr{F}_{2}(V) \rightarrow N(A),
$$

given by $P(v, w)=(v, l(A) w)$ where $v \in V, w \in V \wedge V$. The following diagram is commutative:

$$
\begin{aligned}
& \mathscr{F}_{2}(V) \stackrel{P}{\rightarrow} N(A)- \\
& \downarrow \\
& \mathscr{F}_{2}(V) /\left[\mathscr{F}_{2}(V), \mathscr{F}_{2}(V)\right]=V \underset{\text { identity }}{\rightarrow}
\end{aligned}=N(A) /[N(A), N(A)]
$$

Also, $P$ is a homomorphism of $K$-algebraic groups.

We will call such morphisms $P$ of $\mathscr{F}_{2}(V)$ polarizations of $V$ and denote the set of polarizations of $V$ by $\operatorname{Pol}(V)$. Clearly, the mapping $A \rightarrow P_{A}$ is a bijection between $\operatorname{Alt}(V)$ and $\operatorname{Pol}(V)$.

If $A \in \mathrm{Alt}^{x}(V)$ then all the groups $N(A)$ are isomorphic and we call the abstract group they define the $K$-Heisenberg group over $V$. A given $N(A)$ is then called a presentation of the $K$-Heisenberg group over $V$, which we will sometimes denote by $N_{2 m+1}(K), 2 m=\operatorname{dim}_{K} V$. Notice, the existence of a nontrivial element in $\operatorname{Alt}(V)$ implies that $V$ has even dimension. Also, the 
fixing of an isomorphism of the center of $N_{2 m+1}(K)$ with $K$ is equivalent to a presentation of $N_{2 m+1}(K)$ and when this is done we will also say $N_{2 m+1} K$ is oriented.

An important special case is when $K=\mathbf{R}$. In this case we make the following definition.

Definition. Let $N_{2 m+1}(\mathbf{R})$ be an oriented R-Heisenberg group. An automorphism $J$ of $N_{2 m+1}(\mathbf{R})$ is called a positive definite $C R$-structure if $J$ acts trivially on the center $\mathscr{Z}$ of $N_{2 m+1}(\mathbf{R}), J^{2} \equiv-I$ modulo the center and

$$
[g, J g]>0, \quad g \notin \mathscr{Z}, \quad g \in N_{2 m+1}(\mathbf{R}),
$$

where $[g, h]=g h g^{-1} h^{-1}$ is the usual commutator of $g$ and $h$.

The theory of $C R$-structures on an oriented R-Heisenberg group has been studied in [5] and, loosely speaking, relative to a fixed orientation, it was found that the positive definite $C R$-structures were characterized as those for which a good function theory exists.

We will now examine these concepts in relation to field extensions. Let $K / L$ be a finite field extension of dimension $h$. Consider a vector space $V$ over $K$ and denote by $V(L)$ the inherited vector space structure of $V$ over $L$. If $\operatorname{dim}_{K} V=m$ then $\operatorname{dim}_{L} V=h \cdot m$. Form $\mathscr{F}_{2}(V)$ and $\mathscr{F}_{2}(V(L))$, the free 2-step nilpotent groups over $V$ and $V(L)$, respectively. The identity map $V(L) \hookrightarrow V$ lifts to a homomorphism $i: \mathscr{F}_{2}(V(L)) \rightarrow \mathscr{F}_{2}(V)$. Consider any polarization $P$ of $V$ and let $A$ be the corresponding alternating bilinear form of $V$. The trace mapping $t=\operatorname{tr}_{K / L}: K \rightarrow L$ is a linear mapping of $K$ considered as a vector space over $L$ and hence $B=t \cdot A$ is an alternating bilinear form of $V(L)$. Let $P(L)$ be the corresponding polarization of $V(L)$. Then, where $t$ denotes as well the homomorphism $(v, \alpha) \rightarrow(v, t(\alpha)),(v, \alpha) \in N(A)$ of $N(A)$ onto $N(B)$, we have the commutative diagram

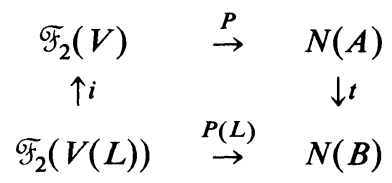

We say that $P$ is an extension of $P(L)$ determined by the extension $K / L$.

A related but distinct concept, loosely called reduction of the scalars, may be considered in the general setting of algebraic group theory. Let $G$ be a $K$-algebraic group and assume that the set of equations defining $G$ can be chosen in the subfield $L$. We say in this case that $G$ is defined over $L$. Suppose, for convenience, that $G \subset G L(V)$ where $V$ is a vector space over $K$. Since every linear transformation of $V$ is a linear transformation of $V(L)$ we have the injective isomorphism

$$
G L(V) \rightarrow G L(V(L))
$$

which we call the isomorphism of reducing the field from $K$ to $L$. Since $G$ is a $K$-algebraic group defined over $L$, we have that $G \subset G L(V(L))$ is an $L$-algebraic group. We call $G$ the $L$-algebraic group obtained by reducing the field from $K$ to $L$.

We will now specialize to the groups consider above. Since $K / L$ is a finite 
extension, the associative algebra representation of $\mathscr{F}_{2}(V)$ is defined over $L$ and hence we may consider the $L$-algebraic group $\mathscr{F}_{2}(V)$. Notice $\mathscr{F}_{2}(V(L)) \neq$ $\mathscr{F}_{2}(V)$. Also if $P$ is any polarization of $V$ and $A$ the corresponding alternating bilinear form of $V$ then the group $N(A)$ is a $K$-algebraic group defined over $L$ and may be considered as an $L$-algebraic group $N(A)$. Notice as well that where $B=t \cdot A$, in the notation considered above, $N(A) \neq N(B)$. Finally, $P$ defines an $L$-algebraic group homomorphism $P$ of $\mathscr{F}_{2}(V)$ onto $N(A)$.

2. The nilpotent groups of an abelian variety. It will be convenient to view complex vector spaces as follows. Let $V$ be a real vector space of even dimension $n=2 n$. A R-linear mapping $J$ of $V$ will be called a complex structure if $J^{2}=-I$. If $J$ is a complex structure on $V$ then the pair $(V, J)$ determines a complex vector space $W$ with $V=W(\mathbf{R})$ and $J$ the automorphism of $W$ given by $w \rightarrow i w$.

Let $(V, J)$ be a complex vector space and $\mathscr{F}_{2}(V)$ the free 2-step nilpotent group over $V$. Then, $J$ induces an automorphism, also denoted by $J$, of $\mathscr{F}_{2}(V)$.

Let $(V, J)$ be a complex vector space. Consider the complex torus $(V / L, J)$, where $L$ is a discrete subgroup of $V$ with $V / L$ compact.

Definition. The complex torus $\mathbf{T}=(V / L, J)$ will be called an abelian variety if it has sufficiently many meromorphic functions to separate points.

Not every complex torus is an abelian variety. We will now discuss circumstances under which $\mathrm{T}$ is an abelian variety. Let $V_{Q}$ be the $Q$-linear span of $L$. Then $V_{Q}$ is the unique rational vector space determined by the conditions $L \subset V_{Q} \subset V$ and $V=V_{Q} \otimes_{Q} \mathbf{R}$. Every bilinear form $B_{Q}$ of $V_{Q}$ extends by linearity to a bilinear form $B$ of $V$. The fundamental theorem of abelian varieties states that $\mathbf{T}=(V / L, J)$ is an abelian variety if and only if there exists an alternating bilinear form $A_{Q}$ of $V_{Q}$ such that the bilinear form of $V$

$$
(x, y) \rightarrow A(J(x), y), \quad x, y \in V,
$$

is positive definite and symmetric.

In [2] this result was reformulated in terms of nilpotent group theory. Firstly, we note that if

$$
P_{Q}: \mathscr{F}_{2}\left(V_{Q}\right) \rightarrow N\left(A_{Q}\right)
$$

is a polarization of $V_{Q}$ then $P_{Q}$ determines a unique polarization $P$ of $V$,

$$
P: \mathscr{F}_{2}(V) \rightarrow N(A),
$$

where $A$ is the linear extension of $A_{Q}$ to $V$. A polarization $P$ of $V$ given in this way will be called a rational polarization. Clearly, this depends upon a fixed choice of a lattice $L$ of $V$ or a rational subspace $V_{Q}$ of $V$ with $V=V_{Q} \otimes_{Q} \mathbf{R}$.

THEOREM. A necessary and sufficient condition for $\mathbf{T}=(V / L, J)$ to be an abelian variety is that there exists a rational polarization $P$ of $V$ such that

(i) The kernel of $P$ in $\mathscr{F}_{2}(V)$ is $J$-invariant.

(ii) The automorphism that $J$ induces on $N(A)$ is a positive definite CR-structure, where $A$ is the alternating bilinear form of $V$ corresponding to $P$. 
Definition. Let $V$ be a real vector space and $L \subset V$ a lattice. A complex structure $J$ on $V$ such that the complex torus $\mathbf{T}=(V / L, J)$ is an abelian variety is called a Riemann matrix for $V / L$. If $\mathbf{A}=(V / L, J)$ is an abelian variety then every rational polarization $P$ which satisfies the above theorem is called a $J$-polarization and $(J, P)$ is called a Riemann pair for $V / L$.

It should be remarked that for a fixed Riemann matrix $J$ of $V / L$ there may be many rational polarizations $P$ such that $(J, P)$ is a Riemann pair. Also, if $P$ is a rational polarization there may be many Riemann matrices $J$ such that $(J, P)$ is a Riemann pair.

The abelian variety $\mathbf{A}=(V / L, J)$ gives rise to the collection of all rational polarizations $P, P: \mathscr{F}_{2}(V) \rightarrow N(A)$, such that $(J, P)$ is a Riemann pair. In particular, we have associated to $\mathbf{A}$ a collection of $\mathbf{R}$-nilpotent algebraic groups $N(A)$ and compact nilmanifolds $N(A) / \Gamma$, where $A$ is the alternating bilinear form on $V$ corresponding to such a polarization $P$ and $\Gamma$ is the group generated by $L$ in $N(A)$. This correspondence has played a role in $[2,5]$ where function theory on $N(A) / \Gamma$ and theta function theory on $\mathbf{A}$ were studied in relationship to each other. However, the abelian variety $\mathbf{A}$ admits additional structure whose understanding will require the construction of polarizations extending those already considered in the sense defined above. In the remaining part of this section we will study this addition structure. Elsewhere, we will apply these ideas to nilpotent group-theoretic analysis of the arithmetic of algebraic number fields.

A linear mapping $f$ of $V$ will be called a morphism of the abelian variety $\mathbf{A}=(V / L, J)$ if $f \cdot J=J \cdot f$ and $f(L) \subset L$. Denote the ring of all morphisms of $\mathbf{A}$ by $\operatorname{End}(A)$ and put

$$
\mathscr{T}(\mathbf{A})=\operatorname{End}(\mathbf{A}) \otimes_{z} Q
$$

We call $\Re(\mathbf{A})$ the rational multiplier algebra of A. It depends solely upon $V_{Q}$ and $J$ and not the lattice $L$ defining $V_{Q}$. Indeed, we may identify $\mathscr{N}(\mathbf{A})=$ $\mathfrak{N}(J)$ with the algebra of linear maps $f$ of $V$ satisfying $f \cdot J=J \cdot f$ and $f\left(V_{Q}\right) \subset V_{Q}$. Denoting the abstract algebra determined by $\Re(\mathbf{A})$ by $\Re$, it is obvious that $\mathfrak{T}$ is a rational associative algebra and

$$
\Re \hookrightarrow \Re(\mathbf{A})_{Q} \subset \operatorname{End}\left(V_{Q}\right) \quad \text { (by restriction) }
$$

is a rational representation of $\mathfrak{T}$.

Consider a rational polarization $P$ of $V / L$ such that $(J, P)$ is a Riemann pair and let $A$ be the corresponding alternating bilinear form of $V$. We shall identify $A \in \operatorname{Alt}(V)$ with $L(A) \in \operatorname{Hom}\left(V, V^{*}\right)$. Then we may regard $A_{Q} \in$ $\operatorname{Hom}\left(V_{Q}, V_{Q}^{*}\right)$. The mapping

$$
M \rightarrow A_{Q}^{-1} M^{*} A_{Q}, \quad M \in \mathscr{N}(\mathbf{A})_{Q}
$$

is a positive involution of $\mathscr{T}(\mathbf{A})_{Q}$ and is called the Rosati involution of $\mathscr{N}(\mathbf{A})_{Q}$ corresponding to $P$. Thus, $\Re$ is a rational associative algebra with a positive involution.

Let us now state a lemma due to Poincaré that will enable us to completely structure $\mathfrak{T}(\mathbf{A})$. 
PoINCARÉ LEMMA. Let $(V / L, J)$ be an abelian variety and take $V_{Q}^{\prime} \subset V_{Q}$, a rational subspace satisfying $V^{\prime}=V_{Q}^{\prime} \otimes_{Q} \mathbf{R} \subset V$ is J-invariant. Then there eixsts $V_{Q}^{\prime \prime} \subset V_{Q}$ such that

(i) $V_{Q}=V_{Q}^{\prime} \oplus V_{Q}^{\prime \prime}$,

(ii) $V^{\prime \prime}=V_{Q}^{\prime \prime} \otimes_{Q} \mathbf{R} \subset V$ is $J$-invariant.

(iii) If $L^{\prime \prime}$ is a lattice in $V_{Q}^{\prime \prime}$ then $\left(V^{\prime \prime} / L^{\prime \prime}, J \mid V^{\prime \prime}\right)$ is an abelian variety.

REMARK. The existence of $V_{Q}^{\prime}$ is equivalent to $\Re(\mathbf{A})$ containing a proper projection operator or idempotent.

We may define $V_{Q}^{\prime \prime}$ as follows. Let $(J, P)$ be a Riemann pair and let $G$ be the subgroup of $N(A)$ generated by $P\left(V^{\prime}\right)$. Let $\mathcal{C}(G)$ denote the centralizer of $G$. Then $\mathcal{C}(G) / \mathscr{Z}$ where $\mathscr{Z}$ is the center of $N(A)$ will be $V^{\prime \prime}$.

There are many technical details to verify, but this is the basis of the argument. (A proof of this assertion can be found in [2].)

We say that $J$ is $Q$-irreducible if $\mathscr{R}(\mathbf{A})$ has no nontrivial projections. Clearly, the Poincaré lemma implies $V=V_{1} \oplus \cdots \oplus V_{l}$ where $J\left(V_{i}\right)=V_{i}$ and $J_{i}=J \mid V_{i}$ is $Q$-irreducible, $i=1, \ldots, l$. We say that $J_{i}$ and $J_{j}$ are $Q$-equivalent if there exists an $M \in \mathfrak{N}(\mathbf{A})$ such that $M: V_{i} \rightarrow V_{j}$ and $M J_{i}=$ $J_{j} M$. We may group together all the $Q$-equivalent $J_{i}$ 's and change the indexing to write

$$
J=\sum_{1}^{l^{\prime}} m_{i} J_{i}, \quad m_{i} \in \mathbf{Z}^{+},
$$

and call $m_{i}$ the multiplicity of $J_{i}$. It follows that

$$
\Re(\mathbf{A})=\oplus \sum_{1}^{l^{\prime}} \Re\left(m_{i} J_{i}\right)
$$

where $\mathscr{R}\left(m_{i} J_{i}\right)$ is the rational multiplication algebra corresponding to $m_{i} J_{i}$ in the above decomposition. Now, it is easily seen that if $J_{i}$ is irreducible then $\mathscr{R}\left(J_{i}\right)$ is a representation $\rho_{i}$ of a division algebra $\mathscr{D}_{i}$ and $\mathscr{R}\left(m_{i} J_{i}\right)$ is the $m_{i} \times m_{i}$ matrix algebra over $\rho_{i}\left(\mathscr{D}_{i}\right)$.

These considerations led Albert to study those abelian variety $\mathbf{A}$ for which $\mathfrak{T}(\mathbf{A})$ is a rational division algebra admitting positive involution. In particular, he studied the following algebraic problems:

(1) Determine the set of all rational division algebras $\mathscr{D}$ with positive involution.

(2) For each such $\mathscr{D}$ determine the set of all positive involutions.

We will pull the algebraic results out of the hat whenever we need them since there are many good expositions of this starting with Albert's [1].

Let $\mathscr{D}$ be a rational division algebra with positive involution and the center of $\mathscr{D}$. An involution $\sigma$ of $\mathscr{D}$ necessarily defines an automorphism of $\mathfrak{f}$. We say that $\sigma$ is of the first kind if $\left.\sigma\right|^{\mathfrak{f}}=$ identity mapping and of the second kind otherwise. Albert proved that $\mathscr{D}$ had to belong to one of the following three classes.

(i) $\mathscr{D}=\mathfrak{f}$ a totally real algebraic number field. In this case the only positive involution is the identity mapping. 
(ii) $\mathscr{D}=\mathbf{K}$ a quaternion division algebra central over a totally real algebraic number field. The positive involutions are necessarily all of the first kind.

(iii) $\mathscr{Q}=\vartheta$ a cyclic division algebra whose center $\mathfrak{t}$ is a totally imaginary quadratic extension of a totally real algebraic number field. The positive involutions are necessarily all of the second kind.

We will now use the rational multiplication algebra $\Re(\mathbf{A})$ of an abelian variety A to 'extend' the polarizations previously considered. To be consistent with the notation of the following sections, we shall denote real vector spaces by $V_{\mathbf{R}}$ and all other structures over $\mathbf{R}$ in a similar manner.

Let $\mathbf{A}=\left(V_{\mathbf{R}} / L, J\right)$ be an abelian variety and suppose its rational multiplication algebra $\mathscr{R}(\mathbf{A})$ is a rational division algebra $\mathscr{Q}_{Q}$. Denote the corresponding rational representation by $\rho_{Q}: \mathscr{D}_{Q} \rightarrow \mathscr{N}(\mathbf{A})_{Q}$. Each rational polarizatioin $P_{\mathbf{R}}$ of $V_{\mathbf{R}} / L$ such that $\left(J, P_{\mathbf{R}}\right)$ is a Riemann pair determines a Rosati involution

$$
M \rightarrow A_{Q}^{-1} M^{*} A_{Q}
$$

of $\Re(\mathbf{A})_{Q}$, when $A_{\mathbf{R}}$ is the alternating bilinear form of $V_{\mathbf{R}}$ corresponding to $P_{\mathbf{R}}$, and hence a positive involution $\tau$ of $\mathscr{D}_{Q}$. Let $\mathfrak{f}_{0}$ be the fixed field of $\tau$ in $\mathfrak{t}$. Then $V_{Q}$ has the structure of a vector space $V$ over $\mathfrak{f}_{0}$ induced by the action of $\Re(\mathbf{A})_{Q}$ on $V_{Q}$ and $\rho_{Q}$ induces a representation $\rho$ of $\mathscr{Q}_{Q}$ considered as an algebra over $\mathfrak{f}_{0}$ on the vector space $V$ over $\mathfrak{f}_{0}$. In the following sections we will prove that there exists a polarization $P^{\prime}$ of $V$, or equivalently, a $B \in \operatorname{Alt}(V)$, such that the following diagram commutes.

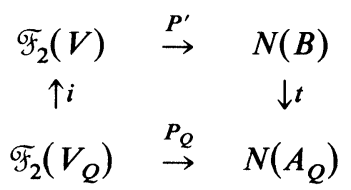

Thus, $A_{Q}=t \circ B$ where $t=\operatorname{tr} \mathfrak{f}_{0} / Q$.

3. Statement of program. Let $\mathscr{D}_{Q}$ be a rational division algebra with positive involution and $\rho_{Q}$ a rational representation of $\mathscr{D}_{Q}$ on a rational vector space $U=U(Q)$. We want to describe the set of complex structures $J$ on $U_{\mathbf{R}}=U(Q) \otimes_{Q} \mathbf{R}$ such that $\rho_{Q}\left(\mathscr{Q}_{Q}\right) \subset \mathscr{N}(J)$ and for each such $J$ we want to determine the rational polarizations $P_{\mathbf{R}}$ of $U_{\mathbf{R}}$ such that $\left(J, P_{\mathbf{R}}\right)$ is a Riemann pair. As indicated above, the problem lives not over $Q$ but over a totally real subfield of the center $\mathfrak{f}^{\prime}$ of $\mathscr{D}_{Q}$.

The plan of attack is loosely speaking that found in [4]. We will find the set of all polarizations $P_{Q}$ of $U(Q)$ which are candidates for a Rosati involution; namely if $A_{Q}$ is the corresponding alternating bilinear form on $U(Q)$ to such a polarization $P_{Q}$ then the mapping $M \rightarrow A_{Q}^{-1} M^{*} A_{Q}$ of $\operatorname{End}(U(Q))$ induces a positive involution of $\mathscr{D}_{Q}$. This will be done in the following steps. Firstly, fix a positive involution $\tau$ of $\mathscr{D}_{Q}$. Consider the set $\mathcal{Q}\left(\mathscr{D}_{Q}, \rho_{Q}, \tau\right)$ of all polarizations $P_{Q}$ of $U(Q)$ for which the mapping $M \rightarrow A_{Q}^{-1} M^{*} A_{Q}$, induces a positive involution of $\mathscr{Q}_{Q}$ coinciding with $\tau$ on the center. Clearly, it is only in case (3) that we need introduce $\tau$ beforehand. Letting $\mathscr{D}$ denote the division algebra 
over $\mathfrak{f}_{0}$ determined by $\mathscr{D}_{Q}$ and $\rho$ the representation of $\mathscr{D}$ on the vector space $U$ over $\mathfrak{f}_{0}$ determined by the action of $\rho_{Q}\left(\mathfrak{f}_{0}\right)$ on $U(Q)$, we set $\left.\mathbb{Q}(\mathscr{Q}), \rho, \tau\right)$ equal to the polarizations $P$ of $U$ such that the mapping $M \rightarrow A^{-1} M^{*} A, M \in \rho(D)$ induces a positive involution coinciding with $\tau$ on the center. We will show that in a natural way $\mathscr{Q}\left(\mathscr{W}_{Q}, \rho_{Q}, \tau\right)$ and $\mathscr{Q}(\mathscr{D}, \rho, \tau)$ are bijectively equivalent. Indeed, this correspondence is given by the last diagram of the preceding section.

The problem of describing $\mathscr{Q}(\mathscr{Q}), \rho, \tau)$ depends ultimately upon knowledge of the group representation $P$ of $\mathscr{Q}^{x}$ determined by $\rho$; namely

$$
P(\delta) X=\rho(\delta)^{*} X \rho(\delta), \quad \delta \in \mathscr{D}^{x}, \quad X \in \operatorname{Hom}\left(U, U^{*}\right) .
$$

Finally, for each $P_{Q} \in \mathbb{Q}\left(\mathscr{D}_{Q}, \rho_{Q}, \tau\right)$ we will produce one Riemann matrix $J=J\left(P_{\mathbf{R}}\right)$ and then all which satisfy

(i) $\left(J, P_{\mathbf{R}}\right)$ is a Riemann pair.

(ii) $\rho_{Q}\left(\mathscr{D}_{Q}\right) \subset \mathscr{T}(\mathbf{J})$.

4. Riemann matrices whose multiplication algebras are totally real fields. The simplest examples of division algebras with positive involution are the totally real fields. Indeed, the identity mapping is the unique positive involution for those fields.

For the remainder of this section, will denote a totally real field and $h=[\mathfrak{f}: Q]$ will be its dimension considered as a vector space over $Q$. Recall, this is equivalent to the existence of $h$ distinct isomorphisms of $\mathfrak{t}$ into $\mathbf{R}$. Denote these isomorphisms by $\chi_{1}, \ldots, \chi_{h}$. Considered as an algebra over $Q$, $t$ has a unique irreducible rational representation, namely the regular representation

$$
r: \mathfrak{f} \rightarrow \operatorname{End}_{Q}(\mathfrak{f})
$$

given by $r(x)(y)=y \cdot x, x, y$ in ${ }^{f}$. Every rational representation $\rho$ of $t$ is the direct sum of copies of the regular representation $r$. We will write $\rho=q \cdot r$ to denote that $\rho$ is the direct sum of $q$ copies of $r$. All this is meant up to rational equivalence. Letting $V$ be a $Q$-vector space and setting $V_{\mathbf{R}}=V \otimes_{Q} \mathbf{R}$, every endomorphism $X$ of $V$ extends uniquely to an endomorphism $X_{\mathbf{R}}$ of $V_{\mathbf{R}}$. In particular, $r(\mathfrak{f})_{\mathbf{R}} \subset \operatorname{End}\left(\mathfrak{f}_{\mathbf{R}}\right)$ and we recall the elementary result that $r(\mathfrak{f})_{\mathbf{R}}$ is diagonalizable. Specifically, a basis of $\mathfrak{f}_{\mathbf{R}}$ can be found relative to which the corresponding matrix representation of $\mathfrak{f}^{\mathrm{determined}}$ by $\boldsymbol{r}$ is given by

$$
x \rightarrow\left(\begin{array}{ccc}
x_{1}(x) & & \\
& \ddots & 0 \\
0 & & x_{h}(x)
\end{array}\right), \quad x \in \mathfrak{f} .
$$

Consider now a fixed rational representation $\rho=q \cdot r$ of $\mathfrak{f}$ on the vector space $V=V(Q)$ over $Q$. The dimension of $V(Q)$ is clearly $h \cdot q$. The representation $\rho$ of $\mathfrak{f}$ determines a vector space structure on $V$ over $\mathfrak{f i v e n}$ by setting

$$
\alpha \cdot v=\rho(\alpha) v, \quad \alpha \in \mathfrak{l}, \quad v \in V .
$$


We denote the resulting vector space over by $V(\mathfrak{t})$. The dimension of $V(\mathfrak{f})$ is $q$. We will now solve the problem of determining all the polarizations of $V(Q)$ that induce the identity involution on $\mathfrak{f}$. We denote this set by $\mathscr{Q}(\mathfrak{f}, \rho)$. In general, for an arbitrary vector space $W$ over a field $K$ and $A \in \operatorname{Alt}(W)$ we let $\pi(A)$ be the polarization

$$
\pi(A): \mathscr{F}_{2}(W) \rightarrow N(A)
$$

of $\mathscr{F}_{2}(W)$ corresponding to $A$.

Let $A \in \operatorname{Alt}(V(\mathfrak{f}))$ and $\pi=\pi(A)$ the corresponding polarization of $\mathscr{F}_{2}(V(\mathfrak{f}))$. Let $t: \mathfrak{f} \rightarrow Q$ be the trace mapping of $\mathfrak{f}$ over $Q$ defined by $t(\alpha)=$ $\operatorname{tr}(r(\alpha))$ where $\operatorname{tr}$ denotes the usual trace of the endomorphism $r(\alpha)$ of considered as a vector space over $Q$. Then $B=t \circ A$ is an element of $\operatorname{Alt}(V(Q))$. Denote by $\pi^{\prime}=\pi(B)$ the corresponding polarization of $F_{2}(V(Q))$. For $x, y \in V(\mathfrak{f})$ and $\alpha \in \mathfrak{f}$ we have

$$
\begin{aligned}
\rho^{*}(\alpha) B(x, y) & =B(x, \alpha y)=t(A(x, \alpha y))=t(\alpha A(x, y)), \\
B \rho(\alpha)(x, y) & =B(\alpha x, y)=t(A(\alpha x, y))=t(\alpha A(x, y))
\end{aligned}
$$

since $A$ is t-invariant

$$
B \rho(\alpha)=\rho^{*}(\alpha) B
$$

and $\pi^{\prime}=\pi(B)$ is in $\mathcal{Q}(\mathfrak{f}, \rho)$. For $\pi=\pi(A)$ in $\operatorname{Pol}(V(\mathfrak{l}))$ we put $t(\pi)=\pi^{\prime}=$ $\pi(B), B=t \circ A$. It follows that

$$
t(\operatorname{Pol}(V(k))) \subset \mathbb{Q}(k, \rho) .
$$

The reverse inclusion lies deeper. To prove it, let $B \in \operatorname{Alt}(V(Q))$ be such that $\pi^{\prime}=\pi(B) \in \mathbb{Q}(\mathfrak{f}, \rho)$. By definition, $\rho^{*}(\alpha) B=B \rho(\alpha)$, whenever $\alpha \in \mathfrak{f}$. This is equivalent to the condition $B(\alpha x, y)=B(x, \alpha y)$, whenever $\alpha \in \mathfrak{f}, x, y \in$ $V(Q)$. Let $v_{1}, \ldots, v_{q}$ be a basis of $V(\mathfrak{f})$ and consider the $Q$-linear mappings

$$
\theta_{i, j}: \alpha \rightarrow B\left(\alpha v_{i}, v_{j}\right), \quad \alpha \in \mathfrak{t}, \quad 1 \leqslant i, j \leqslant q .
$$

The nonsingularity of the trace form implies the existence of elements $\xi_{i, j} \in \mathfrak{t}$ satisfying

$$
B\left(\alpha v_{i}, v_{j}\right)=t\left(\xi_{i, j} \alpha\right), \quad 1 \leqslant i, j \leqslant q .
$$

Since $B(\alpha x, y)=B(x, \alpha y)$ and $B$ is alternating, we have $\xi_{i, j}=-\xi_{j, i}$. Then for $x=\sum_{i=1}^{q} x_{i} v_{i}$ and $y=\sum_{i=1}^{q} y_{i} v_{i}, x_{i}, y_{i} \in \mathfrak{l}$, we have

$$
B(x, y)=t\left(\sum_{1<i<j<q} \xi_{i, j}\left(x_{i} y_{j}-x_{j} y_{i}\right)\right) \text {. }
$$

Let $A \in \operatorname{Alt}(V(\mathfrak{f}))$ be defined by

$$
A(x, y)=\left(\sum_{1<i<j<q} \xi_{i, j}\left(x_{i} y_{j}-x_{j} y_{i}\right)\right) .
$$

We see that $B=t \circ A$. Thus, the converse is verified and we have shown that

$$
\mathbb{Q}(\mathfrak{f}, \rho)=t(\operatorname{Pol}(V(\mathfrak{f}))) \text {. }
$$

We can express this correspondence using the two homomorphisms $i: \mathscr{F}_{2}(V(Q)) \rightarrow \mathscr{F}_{2}(V(\mathfrak{f}))$ and $t: N(A) \rightarrow N(B)$ discussed earlier in terms of the 
following important commutative diagram:

$$
\begin{array}{ccc}
\mathscr{F}_{2}(V(\mathfrak{f})) & \rightarrow & N(A) \\
\uparrow i & & \downarrow t \\
\mathscr{F}_{2}(V(Q)) & \rightarrow & N(B)
\end{array} \quad \pi=\pi(A), \quad \pi^{\prime}=\pi(B), \quad B=t \circ A .
$$

Let $\rho$ be a rational representation of $\mathfrak{f}$ on the vector space $V=V(Q)$ over $Q$ and let $\pi^{\prime}=\pi(B) \in \mathbb{Q}(\mathfrak{f}, \rho)$. Let $\pi=\pi(A) \in \operatorname{Pol}(V(\mathfrak{f}))$ be the unique polarization satisfying the commutative diagram. We shall now construct the 'real closure' of this diagram. Since $\mathcal{F}_{2}(V(Q))$ and $N(B)$ are rational algebraic groups, we may consider the group of real points of these algebraic groups which may be identified with $\mathscr{F}_{2}\left(V_{\mathbf{R}}\right)$ and $N\left(B_{\mathbf{R}}\right)$ respectively. As always $B_{\mathbf{R}}$ is the $\mathbf{R}$-linear extension to an alternating $\mathbf{R}$-bilinear form on $V_{\mathbf{R}}$. We will denote by $\pi_{\mathbf{R}}^{\prime}$ the extension of $\pi^{\prime}$ to $\mathscr{F}_{2}\left(V_{\mathbf{R}}\right)$ or equivalently $\pi_{\mathbf{R}}^{\prime}=\pi\left(B_{\mathbf{R}}\right)$. We saw that $\mathscr{F}_{2}(V(\mathfrak{f}))$ and $N(A)$ are the Hence, by reducing the field to $Q$, we may form rational algebraic groups which are isomorphic to $\mathscr{F}_{2}(V(\mathfrak{t}))$ and $N(A)$, respectively. Denote the group of real points of these rational algebraic groups by $\mathscr{F}_{2}(V(\mathfrak{t}))_{\mathbf{R}}$ and $N(A)_{\mathbf{R}}$ respectively. The construction of $N(A)_{\mathbf{R}}$ can be explicitly described as follows. The group $N(A)$ considered as a rational algebraic group is given as a set by $V(Q) \times \mathfrak{t}(Q)$, where $\mathfrak{f}(Q)$ indicates we are considering $\mathfrak{t}$ as actor space over $Q$. Then $N(A)_{\mathbf{R}}$ as a set is $V_{\mathbf{R}} \times \mathfrak{f}_{\mathbf{R}}$ and the group multiplication is given by the R-linear extension of $A: V(Q) \times V(Q) \rightarrow \mathfrak{f}(Q)$ to $A_{\mathbf{R}}: V_{\mathbf{R}} \times V_{\mathbf{R}} \rightarrow \mathfrak{l}_{\mathbf{R}}$. As before we let $\pi_{\mathbf{R}}: \mathscr{F}_{2}(V(\mathfrak{f}))_{\mathbf{R}} \rightarrow N(A)_{\mathbf{R}}$ be the extension of $\pi$. Finally, the mappings $p$ and $t$ have unique extensions as well, which we denote by $i_{\mathbf{R}}$ and $t_{\mathbf{R}}$, and we have the commutative diagram

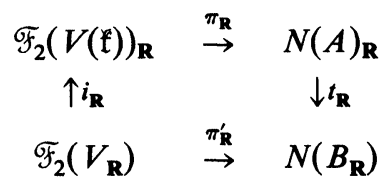

We are now in a position to state and solve our second problem, namely, to find the set of all complex structures $J$ on $V_{\mathbf{R}}$ satisfying the following two conditions.

(i) $\rho_{\mathbf{R}}(\alpha) J=J \rho_{\mathbf{R}}(\alpha), \alpha \in \mathfrak{f}$,

(ii) $\left(J, \pi_{\mathbf{R}}^{\prime}\right)$ is a Riemann pair.

Since the polarization $\pi_{\mathbf{R}}^{\prime}$ of $\mathscr{F}_{2}\left(V_{\mathbf{R}}\right)$ comes from the rational polarization $\pi^{\prime}$ of $\mathscr{F}_{2}(V(Q))$, the content of condition (ii) is that $J$ induces a positive definite $C R$-structure on $N\left(B_{\mathrm{R}}\right)$.

Consider $\rho(\mathfrak{f})$ acting on $N(A)$ by the rule

$$
\rho(\delta)(v, \alpha)=\left(\delta \cdot v, \delta^{2} \alpha\right), \quad \delta, \alpha \in \mathfrak{t}, \quad v \in V .
$$

For each $\delta \in \mathfrak{f}, \rho(\delta)$ is a homomorphism of the group $N(A)$ because $A$ is bilinear over $\mathfrak{f}$. Then we may consider $\rho(\mathfrak{f})$ as homomorphisms on $N(A)$ considered as a rational algebraic group and hence on $N(A)_{\mathbf{R}}$. Also, $\rho_{\mathbf{R}}(\mathfrak{f})=$ $q r_{\mathbf{R}}(\mathfrak{f})$ diagonalizes when considered as endomorphisms of the vector space $V_{\mathbf{R}}$ with eigenvalues given by the isomorphisms $\chi_{i}$ of into $\mathbf{R}$, each with 
multiplicity $q$. Let $N_{2 q+1}\left(\mathbf{R}, \chi_{i}\right)$ be the subgroup of $N(A)_{\mathbf{R}}$ generated by the subspace of $V_{\mathbf{R}} \subset N(A)_{\mathbf{R}}$ corresponding to $\chi_{i}$ under the action of $\rho_{\mathbf{R}}(\mathfrak{f})$. Clearly, $\rho_{\mathbf{R}}(\mathfrak{f})$ extends to a homomorphism $N_{2 q+1}\left(\mathbf{R}, \chi_{i}\right)$, this action being given by

$$
\rho_{\mathbf{R}}(\delta)(v, \alpha)=\left(\delta^{(i)} v, \delta^{(i)^{2}} \alpha\right), \quad \delta^{(i)}=\chi_{i}(\delta) .
$$

It follows that the center of $N_{2 q+1}\left(\mathbf{R}, \chi_{i}\right)$ is 1-dimensional by comparing this action with the action of $\rho_{\mathbf{R}}(\alpha)$ on $N(A)$ above.

It is now easy to see that

(a) $N(A)_{\mathbf{R}}=\Pi_{1}^{h} N_{2 q+1}\left(\mathbf{R}, \chi_{i}\right)$.

(b) $N_{2 q+1}\left(\mathbf{R}, \chi_{i}\right) \cong N_{2 q+1}(\mathbf{R}), i=1, \ldots, h$.

(c) Modulo the center of $N_{2 q+1}\left(\mathbf{R}, \chi_{i}\right)$, the induced action of $\rho_{\mathbf{R}}(\alpha)$ is $v \rightarrow \alpha^{(i)} v$, where $\alpha^{(i)}=\chi_{i}(\alpha)$.

We say this decomposition of $N(A)_{\mathbf{R}}$ is compatible with $\rho(\mathfrak{f})$.

Consider the homomorphism $t_{\mathbf{R}}: N(A)_{\mathbf{R}} \rightarrow N(B)_{\mathbf{R}}$ and the decomposition $N(A)_{\mathbf{R}}=\Pi_{1}^{h} N_{2 q+1}\left(\mathbf{R}, \chi_{i}\right)$ compatible with $\rho(\mathfrak{f})$. The restriction of $t_{\mathbf{R}}$ to $N\left(\mathbf{R}, \chi_{i}\right)$ is an isomorphism of $N\left(R, \chi_{i}\right)$ into $N(B)_{\mathbf{R}}$. Consider $t_{\mathbf{R}}$ on the center of $N\left(\mathbf{R}, \chi_{i}\right)$. Then, under the assumption that $N(B)_{\mathbf{R}}$ is oriented we may orient each $N\left(\mathbf{R}, \chi_{i}\right), i=1, \ldots, h$, by requiring $t_{\mathbf{R}}$ to be orientation preserving. We will assume implicitly throughout that it has been done.

Let $J$ be a complex structure on $V_{\mathbf{R}}$ satisfying the conditions (1) and (2) with respect to $\pi^{\prime} \in \mathbb{Q}(\mathfrak{f}, \rho)$. The endomorphisms $\rho_{\mathbf{R}}(\mathfrak{f})$ of $V_{\mathbf{R}}$ induce homomorphisms of $\mathscr{F}_{2}\left(V_{\mathbf{R}}\right)$ which commute with the automorphism induced by $J$ on $\mathscr{F}_{2}\left(V_{\mathbf{R}}\right)$ by assumption, which implies that $J$ determines an automorphism of $\mathscr{F}_{2}(V(\mathfrak{f}))_{\mathbf{R}}$. To see this, simply show that $J$ preserves the kernel of $\rho_{\mathbf{R}}$. By assumption $J$ also preserves the kernel of $\pi_{\mathbf{R}}^{\prime}$ and hence the kernel of $\mathscr{F}_{2}(V(\mathfrak{f}))_{\mathbf{R}} \rightarrow N(A)_{\mathbf{R}}$ as well. We are identifying all the induced actions of $J$ above under the one symbol $J$ for convenience. Thus, $J$ is an automorphism of $N(A)_{\mathbf{R}}$. Write $N(A)_{\mathbf{R}}=\Pi_{1}^{h} N_{2 q+1}\left(\mathbf{R}, \chi_{i}\right)$. From condition (2) it follows from condition (c), recalling the isomorphisms $\chi_{i}$ are distinct, that the subgroups $N_{2 q+1}\left(\mathbf{R}, \chi_{i}\right)$ are invariant under $J$. Let $J_{i}$ be the restriction of $J$ to $N_{2 q+1}\left(\mathbf{R}, \chi_{i}\right)$. Since $J$ is a positive definite $C R$-structure on $N\left(B_{\mathbf{R}}\right)$ it follows that $J_{i}$ is a positive definite $C R$-structure on $N_{2 q+1}\left(\mathbf{R}, \chi_{i}\right)$. Thus, every $J$ satisfying conditions (1) and (2) with respect to $\pi^{\prime}$ can be written $J=\Pi_{1}^{h} J_{i}$ where $J_{i}$ is a positive definite $C R$-structure on $N_{2 q+1}\left(\mathbf{R}, \chi_{i}\right), i=1, \ldots, h$.

Conversely, if $J_{i}$ is a positive definite $C R$-structure on $N_{2 q+1}\left(\mathbf{R}, \chi_{i}\right), i=$ $1, \ldots, h$, let $J=\pi_{1}^{h} J_{i}$ be the corresponding automorphism of $N(A)_{\mathbf{R}}$. Clearly, $J$ acts trivially on the center of $N(A)_{\mathbf{R}}$ and hence preserves the kernel of $t_{\mathbf{R}}$. This implies we may consider $J$ as an automorphism of $N\left(B_{\mathbf{R}}\right)$. Clearly it is a positive definite $C R$-structure on $N\left(B_{\mathbf{R}}\right)$ which by construction lifts to an automorphism of $\mathscr{F}_{2}\left(V_{\mathbf{R}}\right)$ commuting with $\rho(\mathfrak{f})_{\mathbf{R}}$. It follows that this induced action on $V_{\mathbf{R}}$ is a complex structure on $V_{\mathbf{R}}$ satisfying conditions (1) and (2).

We have proved that if $\mathfrak{G}$ denotes the set of positive definite $C R$-structures on $N_{2 q+1}(\mathbf{R})$ then $\Pi_{1}^{h} \mathscr{S}_{8}$ corresponds to the set of all complex structures $J$ on $V_{\mathbf{R}}$ satisfying conditions (1) and (2) with respect to $\pi^{\prime}$.

5. Representation of involutions: quaternion algebras. Let $\mathscr{D}$ be a rational division algebra having a positive involution of the first kind. By the algebraic 
theory $\mathscr{D}$ is described as follows. Let be the center of $\mathscr{D}$. Then is a totally real field and $h=[\mathfrak{f}, Q]<\infty$. If $\mathscr{D} \neq \mathfrak{f}$ then $\mathscr{D}$ is a quaternion division algebra $\mathbf{K}=\mathbf{K}(a, b)$ over $\mathfrak{t}$ defined as a 4-dimensional vector space over $\mathfrak{t}$

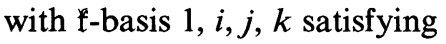

$$
\begin{gathered}
i^{2}=a, \quad j^{2}=b, \quad k^{2}=-a b, \\
i j=-j i=k, \quad j k=-k j=-b i, \quad k i=-i k=-a j
\end{gathered}
$$

where $a, b \in \mathfrak{t}$.

Let $L$ be a subfield of $\mathbf{K}$. We may consider $\mathbf{K}$ as a left-vector space over $L$ or a right-vector space over $L$ where the two structures coincide if $L=\mathfrak{f}$, the center of $\mathbf{K}$. In all cases we shall write linear endomorphisms of $\mathbf{K}$ over $L$ on the left.

Consider $\mathbf{K}$ as a left-vector space over $L$ and take $x \in \mathbf{K}$. The mapping $r(x)$ of $\mathbf{K}$ defined by $r(x)(y)=y \cdot x, y \in \mathbf{K}$ is an endomorphism of $\mathbf{K}$ considered as a left-vector space over $L$. We will write $r(x)$ as $r_{L}(x)$ whenever we are considering the mapping $r(x)$ in this way. The mapping

$$
r_{L}: \mathbf{K} \rightarrow \operatorname{End}_{L}(\mathbf{K})
$$

is called the right-regular representation of $\mathbf{K}$ over $L$. It satisfies $r_{L}(x \cdot y)=$ $r_{L}(y) r_{L}(x), x, y \in \mathbf{K}$, and hence is an algebra homomorphism over $\mathfrak{f}$ of the converse algebra $\mathbf{K}^{\circ}$ into $\operatorname{End}_{L}(\mathbf{K})$.

For $x \in \mathbf{K}$, we put $N_{L}(x)=\operatorname{det} r_{L}(x)$ and since $\mathbf{K}$ is a division algebra it follows that $N_{L}$ is a group homomorphism of $\mathbf{K}^{x}$ into $L^{x}$. We will write $N(x)=N_{L}(x)$, whenever $L$ is a maximal subfield of $\mathbf{K}$. In particular, let $L \mathfrak{f}(i)$, and call $N(x)$ the norm of $x$. Explicitly, if $\delta=x+y i+z j+t k \in \mathbf{K}$ then $N(\delta)=x^{2}-a y^{2}-b z^{2}+a b t^{2}$ and hence $N$ maps $\mathbf{K}^{x}$ into $\mathfrak{l}^{x}$.

Consider now $\mathbf{K}$ as a right-vector space over $L$. To each $x \in \mathbf{K}$, we consider the mapping $l(x)$ of $\mathbf{K}$ defined by $l(x)(y)=x y, y \in \mathbf{K}$, and easily see that $l(x)$ is an endomorphism of $\mathbf{K}$ as a right-vector space of $L$. Considered in this way we write $l(x)$ as $l_{L}(x)$. The mapping

$$
l_{L}: \mathbf{K} \rightarrow \operatorname{End}_{L}(\mathbf{K})
$$

is called the left-regular representation of $\mathbf{K}$. In this case $l_{L}(x y)=l_{L}(x) l_{L}(y)$ and hence $l_{L}$ is an algebra homomorphism over $\mathfrak{f}^{\prime}$ of $\mathbf{K}$ into $\operatorname{End}_{L}(\mathbf{K})$.

In this section and the next, unless otherwise specified, we will consider $\mathbf{K}$ as an algebra over its center $f^{t}$ and write $r$ and $l$ for $r_{\mathfrak{f}}$ and $l_{\mathfrak{f}}$ respectively. From the theory of simple algebras it follows that $r_{Q}$ is an irreducible rational representation of the rational algebra $\mathbf{K}^{\circ}$ and every rational representation of the rational algebra $\mathbf{K}^{\circ}$ is the direct sum of copies of $r_{Q}$. The same theory implies $r$ is an irreducible representation of $\mathbf{K}^{\circ}$ and every representation of $\mathbf{K}^{\circ}$ is the direct sum of copies of $r$. The analogous results hold for $l_{Q}$ and $l$ where $\mathbf{K}^{\circ}$ is replaced by $\mathbf{K}$. The next result holds only when $\mathbf{K}^{\circ}$ is considered as an algebra over $\mathfrak{k}$, being a special case of a result from the theory of central simple algebras. Form the algebra $\mathbf{K} \otimes \mathbf{K}^{\circ}$ and consider the mapping $(\alpha, \beta)$ $\rightarrow l(\alpha) r(\beta)$ of $\mathbf{K} \otimes \mathbf{K}^{\circ}$ into $\operatorname{End}(\mathbf{K})$. The result is that this mapping induces an algebra isomorphism of $\mathbf{K} \otimes \mathbf{K}^{\circ}$ onto $\operatorname{End}(\mathbf{K})$. Hence, the linear span over $\mathfrak{f}$ of the commuting products $l(\alpha) r(\beta)$ is $\operatorname{End}(\mathbf{K})$. 
Let $\mathbf{K}^{*}=\operatorname{Hom}(\mathbf{K}, \mathfrak{f})$ be the vector space dual to $\mathbf{K}$ and consider $\operatorname{Hom}\left(\mathbf{K}, \mathbf{K}^{*}\right)$. For $\gamma \in \mathbf{K}^{*}$ and $X \in \operatorname{Hom}\left(\mathbf{K}, \mathbf{K}^{*}\right)$ put

$$
R(\gamma) X=r^{*}(\gamma) X r(\gamma), \quad L(\gamma) X=l^{*}(\gamma) X l(\gamma)
$$

It is easy to verify that $\mathbf{R}$ is a group representation of $\mathbf{K}^{x}$ on $\operatorname{Hom}\left(\mathbf{K}, \mathbf{K}^{*}\right)$ and $L$ is a group representation of $\left(\mathbf{K}^{\circ}\right)^{x}$ on $\operatorname{Hom}\left(\mathbf{K}, \mathbf{K}^{*}\right)$. For the remainder of this section we will study the representations $R$ and $L$ and representations related to them. In the next section we will use this information to study the theory of Riemann matrices for the quaternion algebras.

Let $\mathbf{K}$ be considered as an algebra over 1 and recall the group homomorphism $N=N_{\mathfrak{t}(i)}: \mathbf{K}^{x} \rightarrow \mathfrak{t}^{x}$ defined above. It is easy to see that there exists a unique involution $\sigma$ of $\mathbf{K}$ of the first kind satisfying the condition $N(\delta)=$ $\delta \sigma(\delta)=\sigma(\delta) \delta$. If $\delta=x+y i+z j+t k \in \mathbf{K}$, then $\sigma(\delta)$ is given by the formula

$$
\sigma(\delta)=x-y i-z j-t k .
$$

Lemma 5.1. Let $T=T^{*}$ be in $\operatorname{Hom}\left(\mathbf{K}, \mathbf{K}^{*}\right)$. Then the following statements are equivalent:

(I) $T(\gamma, \gamma)=T(1,1) N(\gamma)$

(II) $r^{*}(\gamma) \operatorname{Tr}(\gamma)=N(\gamma) T$,

(III) $l^{*}(\gamma) T l(\gamma)=N(\gamma) T$

for all $\gamma \in \mathbf{K}$. We have identified $T$ with its corresponding symmetric bilinear form of $\mathbf{K}$ over $\mathfrak{l}$.

REMARK. The space of all such $T=T^{*} \in \operatorname{Hom}\left(\mathbf{K}, \mathbf{K}^{*}\right)$ which satisfy these conditions is 1 -dimensional and relative to the basis $1, i, j, k$ of $\mathbf{K}$ over $\mathfrak{f}$; one such $T$ is given by the matrix

$$
T=\left(\begin{array}{llll}
1 & & & 0 \\
& -a & & \\
0 & & -b & \\
0 & & & a b
\end{array}\right) .
$$

Proof. Statement (II) and (III) both specialize to statement (I), and hence all we need prove for the first part of this lemma is that (I) implies (II) and (I) implies (III). These proofs are almost identical, hence we shall simply prove (I) implies (II). Thus assume (I). Suppose $T(1,1)=1$ for convenience. Then from

$$
\begin{aligned}
T((\alpha+\beta) \delta,(\alpha+\beta) \delta) & =N((\alpha+\beta) \delta)=N(\alpha+\beta) N(\delta) \\
& =N(\delta) T(\alpha+\beta, \alpha+\beta)
\end{aligned}
$$

we have upon expanding both sides that

$$
T(\alpha \delta, \beta \delta)=N(\delta) T(\alpha, \beta)
$$

or equivalently

$$
r^{*}(\delta) \operatorname{Tr}(\delta)=N(\delta) T .
$$

Suppose now that $T=T^{*} \in \operatorname{Hom}\left(\mathbf{K}, \mathbf{K}^{*}\right)$ satisfies this condition. Then, if 
$\delta=x+y i+z j+t k \in \mathbf{K}$ we have by condition (1)

$$
T(\delta, \delta)=T(1,1) N(\delta)=T(1,1)\left(x^{2}-a y^{2}-b z^{2}+a b t^{2}\right)
$$

which proves our lemma.

We may rewrite conditions (II) and (III) in the following form:

$(\mathrm{II})^{\prime} r^{*}(\delta) T=\operatorname{Tr}(\sigma(\delta))$,

$\left(\right.$ III) ${ }^{\prime} l^{*}(\delta) T=T l(\sigma(\delta))$.

Let us for the remainder of this work let $T$ denote the element defined by Lemma 1. We will use $T$ to analyze the representation $R$ of $\mathbf{K}^{x}$ on $\operatorname{Hom}\left(\mathbf{K}, \mathbf{K}^{*}\right)$. Consider the subspace of $\mathbf{K}$ spanned by $i, j, k$ over $\mathfrak{t}$ and denote it by $\mathbf{K}_{-1}$. It can be characterized as the eigenvalue 1-space of the involution $\boldsymbol{\sigma}$. Further $\mathbf{K}=\mathfrak{1} \oplus \mathbf{K}_{-1}$. We can now write

$$
\begin{aligned}
\operatorname{Hom}\left(\mathbf{K}, \mathbf{K}^{*}\right) & =T \cdot \operatorname{Hom}(\mathbf{K}, \mathbf{K}) \\
& =T \cdot l(\mathbf{K}) \otimes r(\mathbf{K})=T \cdot l(\mathbf{K}) \oplus T \cdot l(\mathbf{K}) \otimes r\left(\mathbf{K}_{-1}\right) .
\end{aligned}
$$

Consider the subspace $W=T \cdot l(\mathbf{K})$. For $T \cdot l(\alpha) \in W$, we have using the commutativity of $r$ and $l$

$$
R(\delta)(T l(\alpha))=r^{*}(\delta) \operatorname{Tl}(\alpha) r(\delta)=\operatorname{Tl}(\alpha) r(\delta \sigma(\delta))=N(\delta) T l(\alpha) .
$$

Hence,

$$
R(\delta) \mid W=N(\delta) \cdot \text { identity mapping. }
$$

Now write $W=W_{A} \oplus W_{S}$, where $W_{A}=T l\left(\mathbf{K}_{-1}\right)$ and $W_{S}=T l(\mathfrak{l})$. Clearly, $W_{S} \subset \operatorname{Sym}(\mathbf{K})$, since $T \in \operatorname{Sym}(\mathbf{K})$. Consider $T l(\alpha) \in T l\left(\mathbf{K}_{-1}\right)$. Then since $\sigma(\alpha)=-\alpha$,

$$
(T l(\alpha))^{*}=l(\alpha)^{*} T=T l(\sigma(\alpha))=-T l(\alpha)
$$

and hence $W_{A} \subset \operatorname{Alt}(\mathbf{K})$. Now consider the subspace $V=T l(\mathbf{K}) \otimes r\left(\mathbf{K}_{-1}\right)$ and write $V=V_{A} \oplus V_{S}$ where $V_{A}=\operatorname{Tr}\left(\mathbf{K}_{-1}\right)$ and $V_{S}=\operatorname{Tl}\left(\mathbf{K}_{-1}\right) \otimes r\left(\mathbf{K}_{-1}\right)$. Arguing as above, it is easy to show that $V_{A} \subset \operatorname{Alt}(\mathbf{K})$ and $V_{S} \subset \operatorname{Sym}(\mathbf{K})$. Consider $V_{A}=\operatorname{Tr}\left(\mathbf{K}_{-1}\right)$. The action of $R(\delta)$ on $\operatorname{Tr}(\alpha) \in V_{A}$ is given as follows,

$$
R(\delta) \operatorname{Tr}(\alpha)=r^{*}(\delta) \operatorname{Tr}(\alpha) r(\delta)=\operatorname{Tr}(\delta \alpha \sigma(\delta)),
$$

which is again in $V_{A}$ since $\sigma(\delta \alpha \sigma(\delta))=\delta \sigma(\alpha) \sigma(\delta)=-\delta \alpha \sigma(\delta) \in \mathbf{K}_{-1}$, whenever $\alpha \in \mathbf{K}_{-1}$. Hence $V_{A}$ is $R$-invariant. Also, since the action of $\mathbf{K}$ on $\mathbf{K}_{-1}$ given by $\alpha \rightarrow \gamma \alpha \sigma(\gamma), \alpha \in \mathbf{K}_{-1}, \gamma \in \mathbf{K}$ is irreducible, it follows that $V_{A}$ is an $R$-invariant and irreducible subspace. Consider $V_{S}=T l\left(\mathbf{K}_{-1}\right) \otimes r\left(\mathbf{K}_{-1}\right)$. Let $\lambda=i, j, k$ in the following. Since $r$ and $l$ commute, it is obvious, from what we have said about $V_{A}$, that each of the spaces $\operatorname{Tr}\left(\mathbf{K}_{-1}\right) l(\lambda)$ is $R$-invariant and irreducible. Hence

$$
V=V_{A} \oplus V_{S}=\operatorname{Tr}\left(\mathbf{K}_{-1}\right) \underset{\lambda=i, j, k}{\oplus} \operatorname{Tr}\left(\mathbf{K}_{-1}\right) l(\lambda)
$$

where the subspaces on the right are 3-dimensional $R$-invariant and irreducible subspaces. We will summarize some of the above discussion in the next two lemmas.

LEMMA 5.2. Let $W=T l(\mathbf{K})$ and $V=T l(\mathbf{K}) \otimes r\left(\mathbf{K}_{-1}\right)$. Then

$$
\operatorname{Hom}\left(\mathbf{K}, \mathbf{K}^{*}\right)=W \oplus V
$$


where $W$ and $V$ are $R$-invariant subspaces satisfying

(I) $R(\delta)$ restricted to $W$ acts as $N(\delta)$ times the identity mapping on $W$ for all $\delta \in \mathbf{K}$.

(II) $V=\bigoplus \Sigma_{\lambda=1, i, j, k} \operatorname{Tr}\left(\mathbf{K}_{-1}\right) l(\lambda)$ where each of the subspaces of the direct sum is $R$-invariant and irreducible.

LemMa 5.3. Let $W$ and $V$ be as in Lemma 2. Then

(i) $\operatorname{Alt}(\mathbf{K})=W_{A} \oplus V_{A}$, where $W_{A}=\operatorname{Tl}\left(\mathbf{K}_{-1}\right)$ and $V_{A}=\operatorname{Tr}\left(\mathbf{K}_{-1}\right)$ are the alternating elements of $W$ and $V$ respectively.

(ii) $\operatorname{Sym}(\mathbf{K})=W_{S} \oplus V_{S}$, where $W_{S}=T l(\mathfrak{f})$ and $V_{S}=T l\left(\mathbf{K}_{-1}\right) \otimes r\left(\mathbf{K}_{-1}\right)$ are the symmetric elements of $W$ and $V$ respectively.

Clearly $W=W_{A} \oplus W_{S}$ and $V=V_{A} \oplus V_{S}$ and we have completely described the representation $R$ of $\mathbf{K}^{x}$ on $\operatorname{Hom}\left(\mathbf{K}, \mathbf{K}^{*}\right)$.

The preceding discussion generalizes as follows. Let $\rho$ be a representation of the algebra $\mathbf{K}^{\circ}$ over $\mathfrak{f}$ on the vector space $U$ over $\mathfrak{f}$. Consider the corresponding group representation $P$ of $\mathbf{K}^{x}$ on $\operatorname{Hom}\left(U, U^{*}\right)$ defined by setting

$$
P(\delta) X=\rho(\delta)^{*} X \rho(\delta), \quad \delta \in \mathbf{K}^{x}, \quad X \in \operatorname{Hom}\left(U, U^{*}\right) .
$$

We can write $\rho=q \cdot r, r$ the regular representation $\mathbf{K}^{\circ}$ and consider the associated decomposition, up to identifications,

$$
U=\oplus \sum_{1}^{q} \mathbf{K}_{u}
$$

where $\mathbf{K}_{u}=\mathbf{K}, u=1, \ldots, q$, and $\rho\left(\mathbf{K}^{\circ}\right) \mid \mathbf{K}_{u}=r\left(\mathbf{K}^{\circ}\right)$. Also, then $U^{*}=$ $\bigoplus \Sigma_{1}^{q} \mathbf{K}_{u}^{*}$ and we can write

$$
\operatorname{Hom}\left(U, U^{*}\right)=\oplus \sum_{u} \operatorname{Hom}\left(\mathbf{K}_{u}, \mathbf{K}_{u}^{*}\right) \oplus \sum_{u<v} B(u, v)
$$

where $B(u, v)=\operatorname{Hom}\left(\mathbf{K}_{u}, \mathbf{K}_{v}^{*}\right) \oplus \operatorname{Hom}\left(\mathbf{K}_{v}, \mathbf{K}_{u}^{*}\right)$. Each of the subspace of the direct sum decomposition are closed under duality and $P$-invariant.

Consider $\operatorname{Sym}(U)$ and let $\delta(u, v) \subset B(u, v)$ be defined by

$$
\delta(u, v)=\left\{X \oplus X^{*}: X \in \operatorname{Hom}\left(\mathbf{K}_{u}, \mathbf{K}_{v}^{*}\right)\right\} .
$$

Clearly we can write

$$
\operatorname{Sym}(U)=\oplus \sum_{u} \operatorname{Sym}\left(\mathbf{K}_{u}\right) \oplus \sum_{u<v} \mathcal{S}(u, v) .
$$

Using Lemma 3 and identifying $\mathbf{K}=\mathbf{K}_{u}$ we have

$$
\operatorname{Sym}\left(\mathbf{K}_{u}\right)=W_{S}(u) \oplus V_{S}(u)
$$

where $W_{S}=W_{S}(u), V_{s}=V_{S}(u)$ and $R\left(\mathbf{K}^{x}\right)$ restricted to $\operatorname{Sym}(\mathbf{K})$ is identical with $P\left(\mathbf{K}^{x}\right)$ restricted to $\operatorname{Sym}\left(\mathbf{K}_{u}\right)$.

Consider $\delta(u, v)$. The mapping $X \rightarrow X \oplus X^{*}$ identifies

$$
\operatorname{Hom}\left(\mathbf{K}, \mathbf{K}^{*}\right)=\operatorname{Hom}\left(\mathbf{K}_{u}, \mathbf{K}_{v}^{*}\right) \rightarrow \varsigma(u, v) \subset B(u, v)
$$

and under this identification the restriction of $P\left(\mathbf{K}^{x}\right)$ to $\delta(u, v)$ coincides with $R\left(\mathbf{K}^{x}\right)$. Hence we may write

$$
\delta(u, v)=W_{s}(u, v) \oplus V_{S}(u, v)
$$


where under the identification above $W=W_{S}(u, v)$ and $V=V_{S}(u, v)$. We have proved the following result except for some simple observations.

THEOREM 5.1. $\operatorname{Sym}(U)=W_{S}(\rho) \oplus V_{S}(\rho)$ where

(1) $W_{S}(\rho)=\Sigma_{u} W_{S}(u) \oplus \Sigma_{u<v} W_{S}(u, v)$ is the subspace of $\operatorname{Sym}(U)$ on which $P(\delta)$ acts by $N(\delta)$ identity, $\delta \in \mathbf{K}^{x}$.

(2) $V_{S}(\rho)=\oplus \Sigma_{u} V_{S}(u) \oplus \Sigma_{u<v} V_{S}(u, v)$ is the direct sum of 3-dimensional $P$-invariant and irreducible subspaces.

It is easy to see that $\operatorname{dim} W_{S}(\rho)=2 q^{2}-q$ and $\operatorname{dim} V_{S}(\rho)=3\left(2 q^{2}+q\right)$. Consider now $\operatorname{Alt}(U)$ which we may write as

$$
\operatorname{Alt}(U)=\oplus \sum_{u} \operatorname{Alt}\left(\mathbf{K}_{u}\right) \oplus \sum_{u<v} \mathfrak{A}(u, v)
$$

where $\mathfrak{A}(u, v)=\left\{X \oplus\left(-X^{*}\right) \in B(u, v): X \in \operatorname{Hom}\left(\mathbf{K}_{u}, \mathbf{K}_{v}^{*}\right)\right\}$. As before the space $\operatorname{Alt}\left(\mathbf{K}_{u}\right)=\operatorname{Alt}(\mathbf{K})$ and hence we may write

$$
\operatorname{Alt}\left(\mathbf{K}_{u}\right)=W_{A}(u) \oplus V_{A}(u)
$$

where $W_{A}=W_{A}(u)$ and $V_{A}=V_{A}(u)$. Also the restriction of $R^{x}\left(\mathbf{K}^{x}\right)$ to $\operatorname{Alt}(\mathbf{K})$ is trivially the restriction of $P\left(\mathbf{K}^{x}\right)$ to $\operatorname{Alt}\left(\mathbf{K}_{u}\right)$. This time the mapping $X \rightarrow X$ $\oplus\left(-X^{*}\right)$ identifies $\operatorname{Hom}\left(\mathbf{K}, \mathbf{K}^{*}\right)=\operatorname{Hom}\left(\mathbf{K}_{u}, \mathbf{K}_{v}^{*}\right)$ with $\mathfrak{A}(u, v)$ and under this identification the restriction of $P\left(\mathbf{K}^{x}\right)$ to $\mathfrak{A}(u, v)$ is the same as $R\left(\mathbf{K}^{x}\right)$. Hence

$$
\mathfrak{U}(u, v)=W_{A}(u, v) \oplus V_{A}(u, v)
$$

where $W=W_{A}(u, v)$ and $V=V_{A}(u, v)$ under the identification.

THEOREM 5.2. Alt $(U)=W_{A}(\rho) \oplus V_{A}(\rho)$ where

(1) $W_{A}(\rho)=\oplus \Sigma_{u} W_{A}(u) \oplus \Sigma_{u<v} W_{A}(u, v)$ is the subspace of all $A \in$ $\operatorname{Alt}(U)$ satisfying $P(\delta) A=N(\delta) A$ whenever $\delta \in \mathbf{K}^{x}$.

(2) $V_{A}(\rho)=\oplus \Sigma_{u} V_{A}(u) \oplus \Sigma_{u<v} V_{A}(u, v)$ can be written as the direct sum of 3-dimensional P-invariant and irreducible subspaces and is the subspace spanned by all the 3-dimensional P-invariant and irreducible subspaces.

It is easy to see that $\operatorname{dim} W_{A}(\rho)=2 q^{2}+q$ and $\operatorname{dim} V_{A}(\rho)=3\left(2 q^{2}-q\right)$.

We will for later applications describe $W_{S}(\rho)$ and $W_{A}(\rho)$ in terms of matrices. Since $W_{A}(u) \cong T l\left(\mathbf{K}_{-}\right)$and $W_{A}(u, v) \cong T l(\mathbf{K})$ we have that $W_{A}(\rho)$ consists of all matrices of the form

$$
\left[\begin{array}{ccc}
T & & 0 \\
& \ddots & \\
0 & & T
\end{array}\right]\left(l\left(\delta_{u v}\right)\right), \quad \delta_{u v}=-\sigma\left(\delta_{v u}\right), 1 \leqslant u, v \leqslant q .
$$

From $W_{S}(u) \cong T l(\mathfrak{f})$ and $W_{S}(u, v) \cong T l(\mathbf{K})$ we have that $W_{S}(\rho)$ consists of matrices

$$
\left[\begin{array}{ccc}
T & & 0 \\
& \ddots & \\
0 & & T
\end{array}\right]\left(l\left(\delta_{u v}\right)\right), \quad \delta_{u v}=\sigma\left(\delta_{v u}\right), 1 \leqslant u, v<q .
$$

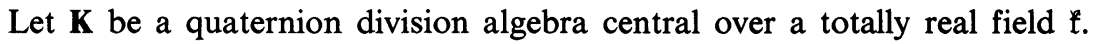
Consider a rational representation $\rho_{Q}$ of $\mathbf{K}$ on a vector space $U=U(Q)$ over

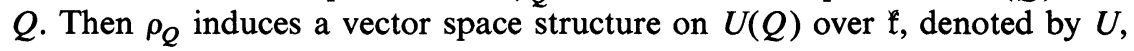


where scalar multiplication is given by $\alpha \cdot u=\rho_{Q}(\alpha) u, \alpha \in \mathfrak{f}, u \in U$. Since $\mathfrak{t}$ is central, $\rho_{Q}$ induces a representation $\rho$ of the algebra $\mathbf{K}$ over $f$ on the vector space $U$. To each positive involution $\tau$ of $\mathbf{K}$ of the first kind we have defined the sets $\mathcal{Q}\left(\mathbf{K}, \rho_{Q}, \tau\right) \subset \operatorname{Pol}(U(Q)) \cong \operatorname{Alt}(U(Q))$ and $\mathbb{Q}(\mathbf{K}, \rho, \tau) \subset \operatorname{Pol}(U) \cong$ $\operatorname{Alt}(U)$. Let $t$ be the trace mapping of $\mathfrak{t}$ over $Q$. Then $t$ induces a mapping, also denoted by $t$, from $\operatorname{Pol}(U)$ into $\operatorname{Pol}(U(Q))$ given as follows. If $A \in$ $\operatorname{Alt}(U)$ then $t(A)=t A \in \operatorname{Alt}(U(Q))$. Arguing as in $\$ 4$ we may show that

$$
t(\mathscr{Q}(\mathbf{K}, \rho, \tau))=\mathbb{Q}\left(\mathbf{K}, \rho_{\mathcal{Q}}, \tau\right)
$$

which as in $\$ 4$ may be expressed by the commutative diagram

$$
\begin{array}{ccc}
\mathscr{F}_{2}(U) & \stackrel{\pi}{\rightarrow} & N(A) \\
\uparrow p & & \downarrow t \\
\mathscr{F}_{2}(U(Q)) & \stackrel{\pi^{\prime}}{\rightarrow} & N(B)
\end{array} \quad \pi=\pi(A) \in \mathbb{Q}(\mathbf{K}, \rho, \tau) .
$$

Hence, the problem of describing $\mathscr{Q}\left(\mathbf{K}, \rho_{Q}\right)=\cup_{\tau>0} \mathscr{Q}\left(\mathbf{K}, \rho_{Q}, \tau\right)$ is the same as that describing $\mathscr{Q}(\mathbf{K}, \rho)=\cup_{\tau>0} \mathscr{Q}(\mathbf{K}, \rho, \tau)$.

We will first solve this problem when $\rho$ is the regular representation $r$ of the algebra $\mathbf{K}^{\circ}$. However, before doing so we will divide the quarternion division algebras over ${ }^{\prime}$ into two distinct classes. The totally positive quaternions, $\mathbf{K}^{+}$, are those $\mathbf{K}(a, b)$ for which $-a$ and $-b$ are totally positive. The totally indefinite quaternions, $\mathbf{K}^{-}$, are those $\mathbf{K}(a, b)$ for which $a,-b$ are totally positive.

For $\mathbf{K}^{+}$, the involution $\sigma$, defined in the preceding section, is the only positive involution while for $\mathbf{K}^{-}, \boldsymbol{\sigma}$ is not a positive involution. However, $\mathbf{K}^{-}$ does have positive involutions of the form

$$
\tau(\delta)=u^{-1} \sigma(\delta) u \quad \delta, u \in \mathbf{K}^{-} \text {and } \sigma(u)=-u .
$$

The complete set of positive involutions of $\mathbf{K}^{-}$can be described as follows. If $\tau_{0}$ is a fixed positive involution of $\mathbf{K}^{-}$then every other positive involution can be written as

$$
\tau(\delta)=u^{-1} \tau_{0}(\delta) u
$$

where $u \in \mathbf{K}^{-}$satisfies

1. $\tau_{0}(u)=u$,

2. $r(u)$ has positive eigenvalues.

We are now ready to describe $\mathscr{Q}\left(\mathbf{K}^{+}, r\right)$ and $\mathscr{Q}\left(\mathbf{K}^{-}, r\right)$.

Consider $\mathscr{Q}\left(\mathbf{K}^{+}, r\right)$. It consists of all those $X \in \operatorname{Alt}(\mathbf{K})$ satisfying $r^{*}(\delta) X=$ $X r(\sigma(\delta))$ for $\delta \in \mathbf{K}$. Multiplying on the right by $r(\delta)$ we have for such $\operatorname{an} X$

$$
r^{*}(\delta) X r(\delta)=X r(\delta \sigma(\delta))=N(\delta) X .
$$

Thus, by Lemma 3 we have

$$
\mathscr{Q}\left(\mathbf{K}^{+}, r\right)=W_{A}=T l\left(\mathbf{K}_{-1}^{+}\right) .
$$

Consider now $\mathscr{Q}\left(\mathbf{K}^{-}, r\right)=\cup_{\tau>0} \mathscr{Q}\left(\mathbf{K}^{-}, r, \tau\right)$. Let $\tau$ be a positive involution of $\mathbf{K}^{-}$and let $u \in \mathbf{K}_{-1}^{-}$satisfy $\tau(\delta)=u \sigma(\delta) u^{-1}$ for all $\delta \in \mathbf{K}^{-}$. We claim

$$
\mathbb{Q}\left(\mathbf{K}^{-}, r, \tau\right)=\operatorname{Tr}(u) l(\mathfrak{f}) .
$$


From $\sigma(u)=-u$ it follows that $(\operatorname{Tr}(u))^{*}=r^{*}(u) T=\operatorname{Tr}(\sigma(u))=-\operatorname{Tr}(u)$ and hence $\operatorname{Tr}(u)$ is alternating. If $\delta \in \mathbf{K}^{-}$then

$$
r^{*}(\delta)(\operatorname{Tr}(u))=\operatorname{Tr}(\sigma(\delta)) r(u)=\operatorname{Tr}(u) r\left(u \sigma(\delta) u^{-1}\right)=\operatorname{Tr}(u) r(\tau(\delta))
$$

implying $\operatorname{Tr}(u) \in \mathbb{Q}\left(\mathbf{K}^{-}, r, \tau\right)$. Conversely if $A \in \mathbb{Q}\left(\mathbf{K}^{-}, r, \tau\right)$ then $\operatorname{Ar}\left(u^{-1}\right)$ is symmetric from

$$
\left(\operatorname{Ar}\left(u^{-1}\right)\right)^{*}=r\left(u^{-1}\right)^{*} A^{*}=-\operatorname{Ar}\left(\tau\left(u^{-1}\right)\right)=\operatorname{Ar}\left(u^{-1}\right)
$$

and in light of Lemma 1

$$
r^{*}(\delta) \operatorname{Ar}\left(u^{-1}\right)=\operatorname{Ar}(\tau(\delta)) r\left(u^{-1}\right)=\operatorname{Ar}\left(u^{-1}\right) r(\sigma(\delta))
$$

implies $\operatorname{Ar}\left(u^{-1}\right) \in T l(\mathfrak{f})$ proving our claim.

We have, from the discussion just completed, that

$$
\mathbb{Q}\left(\mathbf{K}^{-}, r\right) \subset \operatorname{Tr}\left(\mathbf{K}_{-1}^{-}\right)
$$

where $\operatorname{Tr}\left(\mathbf{K}_{-1}^{-}\right)$is the 3-dimensional $R$-invariant and irreducible space considered in Lemma 2. Now let $\tau_{0}$ be a fixed positive involution on $\mathbf{K}^{-}$given by $\tau_{0}(\delta)=u_{0} \sigma(\delta) u_{0}^{-1}, \quad u_{0} \in \mathbf{K}_{-1}^{-}$, and consider $Q\left(\mathbf{K}^{-1}, r, \tau_{0}\right)=\operatorname{Tr}\left(u_{0}\right) l(\mathfrak{f}) \subset$ $\operatorname{Tr}\left(\mathbf{K}_{-1}^{-}\right)$. Take $\gamma \in \mathbf{K}^{-}$and consider

$$
R(\gamma) \operatorname{Tr}\left(u_{0}\right)=r^{*}(\gamma) \operatorname{Tr}\left(u_{0}\right) r(\gamma)=\operatorname{Tr}\left(\gamma u_{0} \sigma(\gamma)\right)
$$

It follows that

$$
R(\gamma) \mathscr{Q}\left(\mathbf{K}_{-1}^{-}, r, \tau_{0}\right)=\mathscr{Q}\left(\mathbf{K}_{-1}^{-}, r, \tau\right)
$$

where $\tau(\delta)=v_{0} \sigma(\delta) v_{0}^{-1}$ and $v_{0}=\gamma u_{0} \sigma(\gamma)$ is a positive involution. The last assertion follows from

$$
\begin{gathered}
\tau(\sigma) \delta=v_{0} \sigma(\delta) v_{0}^{-1} \delta=\gamma\left(\tau_{0}\left(\gamma^{-1} \delta \gamma\right)\left(\gamma^{-1} \delta \gamma\right)\right) \gamma^{-1} \\
\operatorname{trace}(r(\tau(\delta) \delta))=\operatorname{trace}\left(r\left(\tau_{0}\left(\gamma^{-1} \delta \gamma\right) \gamma^{-1} \delta \gamma\right)\right)>0
\end{gathered}
$$

since $\tau_{0}$ was assumed positive. We summarize as follows.

LEMMA 5.4. $\mathcal{Q}\left(\mathbf{K}^{+}, r\right)=T l\left(\mathbf{K}_{-1}^{+}\right)$.

LEMMA 5.5. Let $\tau$ be a positive involution given by $\tau(\delta)=u \sigma(\delta) u^{-1}, u \in \mathbf{K}_{-1}^{-}$. Then

$$
\mathbb{Q}\left(\mathbf{K}^{-}, r, \tau\right)=\operatorname{Tr}(u) l(\mathfrak{f}) .
$$

Moreover

$$
\operatorname{Tr}\left(\mathbf{K}_{-1}^{-}\right)=\sum_{\delta \in \mathbf{K}^{-}} R(\delta) \mathcal{Q}\left(\mathbf{K}^{-}, r, \tau\right)
$$

where $R(\delta) \mathbb{Q}\left(\mathbf{K}^{-}, r, \tau\right)=\mathbb{Q}\left(\mathbf{K}^{-}, r, \tau^{\prime}\right), \tau^{\prime}$ a positive involution.

Consider now the general case where $\rho_{Q}$ is a rational representation of $\mathbf{K}$ on a rational vector space $U=U(Q)$. Let, as discussed, $\rho$ be the representaion of the algebra $\mathbf{K}$ over $\mathfrak{f}$ on the vector space $U$ over $\mathfrak{f}$. We want to describe $\mathbb{Q}(\mathbf{K}, \rho)$.

Assume firstly that $\mathbf{K}=\mathbf{K}^{+}$and hence $\mathscr{Q}(\mathbf{K}, \rho)=\mathbb{Q}(\mathbf{K}, \rho, \sigma)$. Then $\mathscr{Q}(\mathbf{K}, \rho)$ consists of all those polarizations $\pi=\pi(A)$ where $A \in \operatorname{Alt}(U)$ satisfies

$$
\rho(\delta)^{*} A=A \rho(\sigma(\delta)), \quad \delta \in \mathbf{K} \text {. }
$$


This is clearly equivalent to the condition $A \in W_{A}(\rho)$ and hence

$$
\mathscr{Q}\left(\mathbf{K}^{+}, \rho\right)=W_{A}(\rho) .
$$

Before we interpret this result in terms of a commutative diagram we will make several observations. Since $\operatorname{Alt}(U)=(U \wedge U)^{*}$, where $U \wedge U$ is the center of $\mathscr{F}_{2}(U)$, we may, using duality, consider the dual of $W_{A}(\rho) \subset \operatorname{Alt}(U)$ in $U \wedge U$. Specifically

$$
W_{A}(\rho)^{*}=\left\{w \in U \wedge U: W_{A}(\rho) w=0\right\} .
$$

Since $W_{A}(\rho)^{*}$ is central in $\mathscr{F}_{2}(U)$ we may form the group

$$
N\left(\mathbf{K}^{+}, \rho\right)=\mathscr{F}_{2}(U) / W_{A}(\rho)^{*}
$$

and we observe that $\pi \in \mathbb{Q}\left(\mathbf{K}^{+}, \rho\right)$ if and only if $\pi$ induces a homomorphism of $N\left(\mathbf{K}^{+}, \rho\right)$. Hence, the relationship $\mathcal{Q}\left(\mathbf{K}^{+}, \rho_{Q}\right) \cong \mathbb{Q}\left(\mathbf{K}^{+}, \rho\right) \cong W_{A}(\rho)$ can be expressed by the following commutative diagram:

$$
\begin{array}{ccccc}
\mathscr{F}_{2}(U) & \rightarrow & \mathscr{F}_{2}(U) / W_{A}(\rho)^{*}=N\left(\mathbf{K}^{+}, \rho\right) \stackrel{\pi(A)}{\rightarrow} & N(A) & \\
\quad \uparrow i & & \downarrow t & B=t \cdot A . \\
\mathscr{F}_{2}(U(Q)) & \rightarrow & N(B) &
\end{array}
$$

TheOREM 5.3. The space $\mathbb{Q}\left(\mathbf{K}^{+}, \rho_{Q}\right)$ is bijectively equivalent to the space of all polarizations $\pi$ of $\mathscr{F}_{2}(U)$ which factor through the group $N\left(\mathbf{K}^{+}, \rho\right)$. This equivalence is given in the preceding diagram.

Now let $\mathbf{K}^{-}$be a totally indefinite quaternion division algebra and $\tau$ a positive involution given by $\tau(\delta)=u \sigma(\delta) u^{-1}, u \in \mathbf{K}_{-1}^{-}$. Let $\mathbb{Q}(\tau)=$ $\mathcal{Q}\left(\mathbf{K}^{-}, \rho, \tau\right)$. Arguing, exactly as in the special case of the regular representation above we have that

$$
\mathbb{Q}(\tau)=W_{s}(\rho) \rho(u) .
$$

Take $\gamma \in \mathbf{K}^{-}$, then, as before the involution $\tau^{\prime}$ defined by $\tau^{\prime}(\delta)=v \sigma(\delta) v^{-1}$, where $v=\gamma u \sigma(\gamma)$ is a positive involution of $\mathbf{K}^{-}$and $P(\delta) \mathscr{Q}(\tau)=\mathscr{Q}\left(\tau^{\prime}\right)$. Observe we may also write $\tau^{\prime}(\delta)=w \tau(\delta) w^{-1}$ where $w=\gamma \tau(\gamma)$ and $\tau(w)=w$.

Consider any $A=S \rho(u) \in \mathbb{Q}(\tau), S \in W_{s}(\rho)$. Then if $\gamma \in \mathbf{K}^{-}$we have $P(\gamma) A=\rho^{*}(\gamma) S \rho(u) \rho(\gamma)=S \rho(\gamma u \sigma(\gamma))$ and hence $P(\gamma) A \in S \cdot \rho\left(\mathbf{K}_{-1}^{-}\right)$. Since $S \rho\left(\mathbf{K}_{-1}^{-}\right)$is a 3 -dimensional $P$-invariant and irreducible subspace of $\operatorname{Alt}(U)$, it follows that

$$
P(\gamma) A \in S \rho\left(\mathbf{K}_{-1}^{-}\right) \subset V_{A}(\rho), \quad \gamma \in \mathbf{K}^{-} .
$$

In particular, $\mathbb{Q}(\tau) \subset V_{A}(\rho)$ for all positive involutions $\tau$ of $\mathbf{K}^{-}$and $\mathbb{Q}\left(\mathbf{K}^{-}, \rho\right)$ $\subset V_{A}(\rho)$.

Fix the positive involution $\tau_{0}$ and consider $\mathbb{Q}\left(\tau_{0}\right)$. Since $\mathbb{Q}\left(\tau_{0}\right)=$ $W_{s}(\rho) \rho\left(u_{0}\right)$, where $\tau_{0}(\delta)=u_{0}^{+1} \sigma(\delta) u_{0}^{-1}$, we have that $p=\operatorname{dim} \mathbb{Q}\left(\tau_{0}\right)=$ $\operatorname{dim} W_{s}(\rho)=2 q^{2}-q$. Choose a basis $S_{\mu}$ of $W_{s}(\rho), \mu=1, \ldots, p$, and consider the basis $A_{\mu}=S_{\mu} \rho(u), \mu=1, \ldots, p$. Each $A_{\mu}$ is contained in the 
3-dimensional $P$-invariant, irreducible subspace $S_{\mu} \rho\left(\mathbf{K}_{-1}^{-}\right)$. Since $\operatorname{dim} V_{A}(\rho)=$ $3\left(2 q^{2}-q\right)$, it is reasonable to conjecture that

$$
V_{A}(\rho)=\bigoplus \sum_{\mu=1}^{p} S_{\mu} \rho\left(\mathbf{K}_{-1}^{-}\right) .
$$

To prove this, we must show that $\Sigma_{\mu=1}^{p} S_{\mu} \rho\left(\mathbf{K}_{-1}^{-}\right)$is a direct sum. We will do this by induction. Suppose for $n<p$, the sum $\sum_{\mu=1}^{n-1} S_{\mu}\left(\mathbf{K}_{-1}^{-}\right)$is a direct sum and consider $S_{n} \rho\left(\mathbf{K}_{-1}^{-}\right)$. If the sum $\sum_{\mu=1}^{n} S_{\mu} \rho\left(\mathbf{K}_{-1}^{-}\right)$is not a direct sum the irreducibility of $S_{\pi} \rho\left(\mathbf{K}_{-1}^{-}\right)$implies $S_{n} \rho\left(\mathbf{K}_{-1}^{-}\right) \subset \sum_{\mu=1}^{\pi-1} S_{\mu} \rho\left(\mathbf{K}_{-1}^{-}\right)$. Then for any $\delta \in \mathbf{K}_{-1}^{-}, \delta \neq 0$ we have

$$
S_{n} \rho(\delta)=\sum_{\mu=1}^{n-1} S_{\mu} \rho\left(\delta_{\mu}\right), \quad \delta_{\mu} \in \mathbf{K}_{-1}^{-} .
$$

Hence $S_{n}=\sum_{\mu=1}^{n-1} S_{\mu} \rho\left(\delta^{-1} \delta_{\mu}\right)$ and, since $S_{n}=S_{n}^{*}$, it follows that $\sum_{\mu=1}^{n-1} S_{\mu} \rho\left(\delta^{-1} \delta_{\mu}\right)=\sum_{\mu=1}^{n-1} S_{\mu} \rho\left(\sigma\left(\delta^{-1} \delta_{\mu}\right)\right)$. The induction hypothesis implies $\delta^{-1} \delta_{\mu}$ $\in \mathfrak{f}, \mu=1, \ldots, p$, and hence $S_{n}$ is linearly dependent on $S_{1}, \ldots, S_{n-1}$ over $\mathfrak{f}$. This last assertion contradicts our choice of the $S_{\mu}$ and our claim is proved. The next theorem summarizes some of our discussion.

THEOREM 5.4. For every positive $\tau$ of $\mathbf{K}^{-}$we have

$$
\mathscr{Q}\left(\mathbf{K}^{-}, \rho, \tau\right)=W_{s}(\rho) \rho(u) \subset V_{A}(\rho)
$$

where $\tau(\delta)=u \sigma(\delta) u^{-1}, u \in \mathbf{K}_{-1}^{-}$. The space $V_{A}(\rho)$ is spanned over $\mathfrak{f}$ by $\mathbb{Q}\left(\mathbf{K}^{-}, \rho\right)$ and is the smallest $P$-invariant subspace containing any $\mathbb{C}\left(\mathbf{K}^{-}, \rho, \tau\right), \tau$ a positive involution of $\mathbf{K}^{-}$.

Let $\tau$ be a positive involution of $\mathbf{K}^{-}$and consider $\mathscr{Q}(\tau)=\mathscr{Q}\left(\mathbf{K}^{-}, \rho, \tau\right)$. As after Theorem 3, we may view $\mathscr{Q}(\tau) \widetilde{\subset} \operatorname{Alt}(U)=(U \wedge U)^{*}$ and consider $\mathbb{Q}(\rho)^{*}$ defined by

$$
\mathbb{Q}(\tau)^{*}=\{w \in U \wedge U: \mathbb{Q}(\tau) w=0\} .
$$

Since every polarization $\pi \in \mathbb{Q}(\tau)$ induces a homomorphism of the group

$$
N_{\tau}\left(\mathbf{K}^{-}, \rho\right)=\mathscr{F}_{2}(U) / \mathscr{Q}(\tau)^{*}
$$

we have the following result.

THeOReM 5.5. For each positive involution $\tau$ of $\mathbf{K}^{-}$the space $\mathbb{Q}\left(\mathbf{K}^{-}, \rho_{Q}, \tau\right)$ is bijectively equivalent to the space of all polarizations $\pi$ of $\mathscr{F}_{2}(U)$ which factor through the group $N_{\tau}\left(\mathbf{K}^{-}, \rho\right)$. The exact relationship is given by the commutative diagram

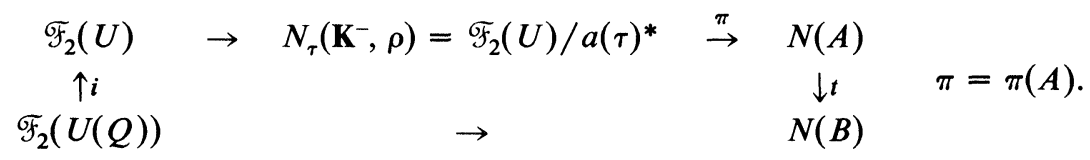


Theorem 4 also implies that if $N\left(\mathbf{K}^{-}, \rho\right)=\mathscr{F}_{2}(U) / V_{A}(\rho)^{*}$ the following diagram commutes for all positive involutions $\tau$ of $\mathbf{K}^{-}$.

$$
\begin{array}{ccc}
\mathscr{F}_{2}(U) & \rightarrow & N\left(\mathbf{K}^{-}, \rho\right) \\
& \searrow & \downarrow \\
& N_{\tau}\left(\mathbf{K}^{-}, \rho\right)
\end{array}
$$

In terms of matrices, $\mathbb{Q}\left(\mathbf{K}^{+}, \rho\right)=W_{A}(\rho)$ has been described in the discussion following Theorem 2. Consider $\mathbf{K}^{-}$and a positive involution $\tau$ of $\mathbf{K}^{-}$and set $T(\tau)=T l(u) r(u)$ where $\tau(\delta)=u \sigma(\delta) u^{-1}$. Then the elements of $\mathcal{Q}\left(\mathbf{K}^{-}, \rho, \tau\right)$ are given in the form

$$
\left[\begin{array}{ccc}
T(\tau) & & \\
& \ddots & 0 \\
0 & & T(\tau)
\end{array}\right)\left(l\left(\delta_{\mu, v}\right)\right), \quad \tau\left(\delta_{\mu v}\right)=-\delta_{v \mu}, \quad 1 \leqslant \mu, v \leqslant q .
$$

6. Riemann matrices: quaternion algebras. In $\S 5$ we obtain a description of $\mathcal{Q}\left(\mathbf{K}, \rho_{Q}, \tau\right)$ where $\mathbf{K}$ is a quaternion division algebra central over a totally real field $\mathfrak{f}$ with positive involution $\tau$ and rational representation $\rho_{Q}$ on the rational vector space $U=U(Q)$. Let $\rho$ be the corresponding representation over $\mathfrak{t}$ of $\mathbf{K}$ on $U=U(\mathfrak{f})$. We expressed the relationship between $\mathbb{Q}(\mathbf{K}, \rho, \tau)$ and $\mathcal{Q}(\mathbf{K}, \rho, \tau)$ in terms of the commutative diagram

$$
\begin{array}{ccc}
\mathscr{F}_{2}(U) & \stackrel{\pi}{\rightarrow} & N(A) \\
\uparrow i & & \downarrow t \\
\mathscr{F}_{2}(U(Q)) & \stackrel{\pi}{\rightarrow} & N(B)
\end{array} \quad \pi \in \mathbb{Q}(\mathbf{K}, \rho, \tau) .
$$

As in $\$ 4$ we may consider the real closure of this diagram

$$
\begin{array}{ccl}
\mathscr{F}_{2}(U)_{\mathbf{R}} & \stackrel{\pi_{\mathbf{R}}}{\rightarrow} & N(A)_{\mathbf{R}}=N\left(A_{\mathbf{R}}\right) \\
\uparrow_{i_{\mathbf{R}}} & & \downarrow t_{\mathbf{R}} \\
\mathscr{F}_{2}\left(U_{\mathbf{R}}\right) & \rightarrow & N(B)_{\mathbf{R}}=N\left(B_{\mathbf{R}}\right)
\end{array}
$$

The problem is to describe for each $\pi^{\prime} \in \mathbb{Q}\left(\mathbf{K}, \rho_{Q}, \tau\right)$ the collection of all complex structures $J$ on $U_{\mathbf{R}}$ which satisfy

$\left(1^{\prime}\right) \rho_{\mathbf{R}}(\alpha) J=J \rho_{\mathbf{R}}(\alpha), \alpha \in \mathbf{K}$.

$\left(2^{\prime}\right)\left(J, \pi_{\mathbf{R}}^{\prime}\right)$ is a Riemann pair.

As in $\$ 4$ we may decompose $N(A)_{\mathbf{R}}$ into a direct product compatible with the action of $\rho(\mathfrak{f})$. Precisely, let $\rho_{\mathbf{R}}(\mathfrak{f})=q \cdot r_{\mathbf{R}}(\mathfrak{f})$ and define $N_{8 q+1}\left(\mathbf{R}, \chi_{i}\right)$ to be the subgroup of $N(A)_{\mathbf{R}}$ generated by the subspace of $U_{\mathbf{R}}$ corresponding to the 'character' $\chi_{i}$ of $\operatorname{tin} \mathbf{R}$. Then

(a') $N(A)_{\mathbf{R}}=\Pi_{1}^{h} N_{8 q+1}\left(\mathbf{R}, \chi_{i}\right)$.

(b') $N_{8 q+1}\left(\mathbf{R}, \chi_{i}\right)$ is isomorphic to $N_{8 q+1}(\mathbf{R}), i=1, \ldots, h$.

(c') Modulo the center of $N_{8 q+1}(\mathbf{R})$, the restriction of $\rho_{\mathbf{R}}(\mathfrak{f})$ is given by the scalar action determined by $\chi_{i}, i=1, \ldots, h$. 
It follows, exactly as before, that $R\left(K, \rho_{Q}, B\right)$, the collection of all complex structures on $U_{\mathbf{R}}$ satisfying conditions $\left(1^{\prime}\right)\left(2^{\prime}\right)$, is given by

$$
\mathscr{R}\left(\mathbf{K}, \rho_{Q}, B\right)=\prod_{1}^{h} \mathfrak{S}_{i}(A)
$$

where $\mathfrak{Q}_{i}(A)$ is the set of all positive definite $C R$-structures $J_{i}$ on $N_{8 q+1}\left(\mathbf{R}, \chi_{i}\right)$ such that the induced action of $J_{i}$ on $U_{\mathbf{R}}=N_{8 q+1}\left(\mathbf{R}, \chi_{i}\right) /$ center commutes with $\rho(\mathbf{K})$, elementwise.

We shall now find elements in $\Re\left(K, \rho_{Q}, B\right)$. The first case we consider is where $\rho_{Q}=r_{Q}$. Again, we break the discussion with two cases.

Let $\mathbf{K}^{+}$be a totally positive quaternion division algebra, $B \in \mathbb{Q}\left(\mathbf{K}^{+}, r_{Q}\right)$ and $A$ the corresponding element in $\mathcal{Q}\left(\mathbf{K}^{+}, r\right)$, with $B=t \cdot A$, where $r$ denotes the right regular representation of $\mathbf{K}^{+}$over its center $\mathfrak{f}$. Then we may write $A=T l\left(\delta_{0}\right), \sigma\left(\delta_{0}\right)=-\delta_{0}$. Observe that $T$ is totally positive definite, i.e., $T(\gamma, \gamma)$ is totally positive in $\mathfrak{f}, \gamma \neq 0$, since $\mathbf{K}^{+}$is totally positive. Consider $N\left(\delta_{0}\right)=T\left(\delta_{0}, \delta_{0}\right)$. It has a totally positive square root in the algebra $\otimes_{Q} \mathbf{R}$ which, by $\mathbf{R}$-linear extension, acts on $U_{\mathbf{R}}=\mathbf{K}_{\mathbf{R}}^{+}$. Thus, we can define the automorphism $J$ of $N(A)_{\mathbf{R}}$ by

$$
(\delta, \gamma) \rightarrow\left(\frac{\delta_{0}}{\sqrt{N\left(\delta_{0}\right)}} \delta, \gamma\right), \quad(\delta, \gamma) \in N(A)_{\mathbf{R}}
$$

That this is an automorphism follows from the formula

$$
l\left(\delta_{0}\right) * A l\left(\delta_{0}\right)=N\left(\delta_{0}\right) A .
$$

Since $\delta_{0}^{2}=-N\left(\delta_{0}\right), J^{2}=-I$ modulo the center, and acts by the identity mapping on the center. Now take $\delta \in \mathbf{K}^{+}$satisfying $r(\gamma) \delta=\chi_{i}(\gamma) \delta$ for fixed $i=1, \ldots, h$ and for all $\gamma \in \mathbb{f}$. To see that the restriction of $J$ to the groups $N_{8 q+1}\left(\mathbf{R}, \chi_{i}\right)$ is a positive definite $C R$-structure, we write $\delta=(\delta, 0) \in N(A)_{\mathbf{R}}$ and note that

$$
[\delta, J \delta]=A(\delta, J \delta)=\sqrt{N\left(\delta_{0}\right)} T(\delta, \delta)
$$

which is totally positive whenever $\delta \neq 0$. Clearly, $J$ commutes with $r(\alpha)$ on $\mathbf{K}_{\mathbf{R}}^{+}$.

The case of $\mathbf{K}^{-}$, a totally indefinite quaternion division algebra, goes as follows. Let $\tau(\delta)=u \sigma(\delta) u^{-1}, \sigma(u)=-u$ be a positive involution of $\mathbf{K}^{-}$and take $B \in \mathbb{Q}\left(\mathbf{K}^{-}, r_{Q}, \tau\right)$ corresponding to $A \in \mathbb{Q}\left(\mathbf{K}^{-}, r, \tau\right)$. We may write $A=$ $\operatorname{Tr}(u) l(\alpha), \alpha \in \mathfrak{f}$. Without loss of generality, we may take $\alpha=1$. Let $v=u^{-1}$. Then $\tau(v)=u^{-1} \sigma(v) u=-u^{-1} u^{-1} u=-u^{-1}=-v$ and $\sigma\left(u^{-1}\right) u^{-1}=\tau\left(u^{-1}\right) u^{-1}=$ $N\left(u^{-1}\right)$ is totally positive on $\mathfrak{f}$, by the assumption of positivity of $\tau$. Consider the mapping $J$ of $N(A)_{\mathbf{R}}$ given by

$$
(\delta, \gamma) \rightarrow\left(\frac{u^{-1}}{\sqrt{N\left(u^{-1}\right)}} \delta, \gamma\right), \quad(\delta, \gamma) \in N(A)_{\mathbf{R}}
$$

It is again easy to see that $J$ is an autormorphism of $N(A)_{\mathbf{R}}$, whose square $J^{2}=-I$ modulo the center and induces, by restriction, to each $N_{8 q+1}\left(\mathbf{R}, \chi_{i}\right)$ a positive definite $\mathbf{C} R$-structure. 
We will now return to the case of a general representation $\rho_{Q}$ of $\mathbf{K}$ on a rational vector space $U=U(Q)$. Let $\rho$ be the corresponding representation of $\mathbf{K}$ over $\mathfrak{t}$ on the vector space $U=U(\mathfrak{l})$ over $\mathfrak{f}$. One of the advantages of introducing the groups $N(A)$ and $N(A)_{\mathbf{R}}$ for an $A \in \mathbb{Q}(\mathbf{K}, \rho, \tau)$ is that $\rho\left(\mathbb{f}^{x x}\right)$ extends to a group automorphisms of $N(A)$ and 'diagonalizes' as a group of automorphisms of $N(A)_{\mathbf{R}}$. We shall now see that for arbitrary $\mathbf{K}$ the group generated by some basis of $\mathbf{K}$ over $\mathfrak{t}^{c}$ also acts as a finite group of automorphisms of $N(A)_{\mathbf{R}}$. This in turn will allow us to find a 'Riemann matrix' for $A$.

Consider $\mathbf{K}=\mathbf{K}^{+}$and $A \in \mathbb{Q}\left(\mathbf{K}^{+}, \rho\right)$. Then, since $\sigma$, the standard involution on $\mathrm{K}^{+}$, is positive, we have that for all $\delta \in \mathbf{K}^{+}, \delta \neq 0, \delta \sigma(\delta)=N(\sigma)$ is a totally positive, element in and hence has a totally, positive square root in $\mathfrak{f} \otimes_{Q} \mathbf{R}$. Then for $\delta \in \mathbf{K}^{+}$the mapping

$$
(u, \alpha) \rightarrow\left(\frac{1}{\sqrt{N(\delta)}} \rho(\delta) u, \alpha\right), \quad(u, \alpha) \in N(A)_{\mathbf{R}}
$$

is an automorphism of $N(A)_{\mathbf{R}}$. The group generated by the automorphisms corresponding to $\delta=1, i, j, k$ is clearly a finite group of automorphisms of $N(A)_{\mathbf{R}}$. Notice as well, that, since $\mathfrak{f}$ is central, these automorphisms induce automorphisms on the groups $N_{8 q+1}\left(\mathbf{R}, \chi_{i}\right)$.

In the case $\mathbf{K}=\mathbf{K}^{-}$and $A \in \mathbb{Q}\left(\mathbf{K}^{-}, \rho, \tau\right)$ not all of $\rho\left(\mathbf{K}^{-}\right)$extends to $N(A)$. However, we can do the following. Write $\tau(\delta)=u_{0} \sigma(\delta) u_{0}^{-1}, \sigma\left(u_{0}\right)=u_{0}$. Then $\tau\left(u_{0}\right)=-u_{0}$. Choose $v_{0}$ such that $\sigma\left(v_{0}\right)=-v_{0}, \tau\left(v_{0}\right)=v_{0}$ and $1, u_{0}, v_{0}, u_{0} v_{0}$ span $\mathbf{K}^{-}$. Then $u_{0} \tau\left(u_{0}\right)=-u_{0}^{2}$ and $v_{0} \tau\left(v_{0}\right)=v_{0}^{2}$ are totally positive in $\otimes_{Q} \mathbf{R}$ and, as above, we have the automorphisms of $N(A)_{\mathbf{R}}$,

$$
\begin{aligned}
& (u, \alpha) \rightarrow\left(\frac{1}{\sqrt{-v_{0}^{2}}} \rho\left(u_{0}\right) u, \alpha\right), \\
& (u, \alpha) \rightarrow\left(\frac{1}{\sqrt{v_{0}^{2}}} \rho\left(v_{0}\right) v, \alpha\right),
\end{aligned}
$$

Hence, the group generated by the basis $1, u_{0}, v_{0}, u_{0} v_{0}$ acts on $N(A)_{\mathbf{R}}$ as a finite group of automorphisms. As above the subgroups $N_{8 q+1}\left(\mathbf{R}, \chi_{i}\right)$ are invariant.

Now, take any positive definite $C R$-structure $J_{i}$ on $N_{8 q+1}\left(\mathbf{R}, \chi_{i}\right), i=$ $1, \ldots, h$. In either case the group $O\left(J_{i}\right)$ of automorphisms of $N_{8 q+1}\left(\mathbf{R}, \chi_{i}\right)$ which commute with $J_{i}$ is maximal compact and contains a conjugate of the finite group of automorphisms defined above. Thus, a conjugate of $J_{i}$, which is still a positive definite $C R$-structure, commutes with this finite group and hence on $N_{8 q+1}\left(\mathbf{R}, \chi_{i}\right)$ modulo the center with $\rho(\mathbf{K})$.

7. Representation of involutions: cyclic algebras. We will now study rational division algebras admitting a positive involution of the second kind. Such algebras are necessarily examples of cyclic algebras whose theory we will recall below.

Let $\vartheta$ be a rational algebra having dimension $s^{2}$ over its center $\mathfrak{f}$. Let $K$ be a cyclic extension of $\mathfrak{f}$ contained in $\vartheta$ of degree $s$ over $\mathfrak{f}$ and denote the Galois 
group of $K$ over $\mathfrak{t}$ by $G$. We will call $\vartheta$ a cyclic algebra over $\mathfrak{t}$ and $K$ its splitting field if a basis of $\vartheta$ considered as a left-vector space over $L$ exists of the form

$$
1, j, j^{2}, \ldots, j^{s-1}
$$

satisfying the next two conditions

(i) For each $\alpha \in K$ we have $j \alpha j^{-1} \in K$ and the mapping

$$
\alpha \rightarrow j \alpha j^{-1}: K \rightarrow K
$$

is a generator of $G$.

(ii) $j^{s}=b \in K^{x}$.

For the rest of this section, $\vartheta$ is a rational division algebra having dimension $s^{2}$ over its center $f$ and $\tau$ is a positive involution on $\vartheta$ of the second kind. The positivity of $\tau$ implies that the fixed field $\mathfrak{f}_{0}$ of $\tau$ is a totally real field. It is well known that every rational simple algebra may be realized as a cyclic algebra and hence $\vartheta$ is a cyclic algebra. We may choose a splitting field $K$ for $\vartheta$ having special properties relative to the involution $\tau$. Firstly, 1 is a totally complex quadratic extension of $\mathfrak{f}_{0}$ and a $c \in \mathfrak{l}$ may be found satisfying

(iii) $\mathfrak{f}=\mathfrak{f}_{0}(c)$,

(iv) $\tau(c)=-c$.

Now, we may choose a cyclic extension $K_{0}$ of $\mathfrak{f}_{0}$ of degree $s$ contained in $\vartheta$ with the following properties.

(v) $K_{0}$ is a totally real field.

(vi) $\tau$ acts by the identity mapping on $K_{0}$.

(vii) $K=K_{0}(c)$ is a splitting field for the cyclic algebra $\vartheta$.

We will now suppose that, once and for all, a $c \in \mathfrak{t}$ has been chosen satisfying (iii) and (iv) and a cyclic extension $K_{0}$ of $\mathfrak{f}_{0}$ has been chosen satisfying (v)-(vii) and a basis $i, j, \ldots, j^{s-1}$ of the left vector space $\vartheta$ over $K$ satisfying (i), (ii). We then have the following two important properties.

(viii) $\tau(j) j=a \in K_{0}$ is totally positive.

(ix) $N_{t / t_{0}}(b)=N_{K_{0} / t_{0}}(a)$.

Also, in terms of our given $\tau$ the positive involutions of $\vartheta$ which have the same effect on $f$ as $\tau$ are all of the form $\tau^{\prime}(\delta)=u \tau(\delta) u^{-1}$ where $\tau(u)=u$ and the eigenvalues of the regular representation of $\vartheta$ applied to $u$ are all positive.

Consider a rational representation $\rho_{Q}$ of $\vartheta^{0}$ on a rational vector-space $U=U(Q)$. Then as we saw in $\S 4, \rho_{Q}$ determines a vector-space structure on $U$ over $\mathfrak{f}_{0}$, which we denote by $U$ again, where scalar multiplication is given by $\alpha \cdot u=\rho_{Q}(\alpha) u, \alpha \in \delta_{0}, u \in U$. Since $\mathfrak{f}_{0}$ is central in $\vartheta^{0}, \rho_{Q}(\vartheta)$ consists of endomorphisms of $U$ and hence $\rho_{Q}$ induces a representation of the algebra $\vartheta$ over $\mathfrak{l}_{0}$ on $U$ which we will denote by $\rho$. To each positive involution $\tau^{\prime}$ on $\vartheta$ we have defined the sets

$$
\begin{aligned}
\mathscr{Q}\left(\vartheta, \rho_{Q}, \tau^{\prime}\right) \subset \operatorname{Pol}(U(Q)) & \cong \operatorname{Alt}(U(Q)), \\
\mathcal{Q}\left(\vartheta, \rho, \tau^{\prime}\right) \subset \operatorname{Pol}(U) & \simeq \operatorname{Alt}(U)
\end{aligned}
$$

and where $t$ now denotes the trace mapping of $\mathfrak{f}_{0}$ into $Q, t \cdot \operatorname{Alt}(U) \subset$ $\operatorname{Alt}(U(Q))$. As in $\$ 4$, we can in fact show

$$
t\left(\mathscr{Q}\left(\vartheta, \rho, \tau^{\prime}\right)\right)=\mathscr{Q}\left(\vartheta, \rho_{Q}, \tau^{\prime}\right)
$$


and we have the commutative diagram

$$
\begin{array}{ccc}
\mathscr{F}_{2}(U) & \rightarrow & N(A) \\
\uparrow p & & \downarrow t \\
\mathscr{F}_{2}(U(Q)) & \stackrel{\pi^{\prime}}{\rightarrow} & N(B)
\end{array}
$$

Thus, we are led to a study of the set $\mathscr{Q}\left(\vartheta, \rho, \tau^{\prime}\right)$. We will write $\tau^{\prime} \sim \tau$ to mean $\tau$ and $\tau^{\prime}$ coincide on $\mathfrak{f}$ and we are interested in the sets $\mathscr{Q}(\vartheta, \rho, \tau)$ and $\mathcal{Q}(\vartheta, \rho)=\cup_{\tau^{\prime} \sim \tau} \mathcal{Q}\left(\vartheta, \rho, \tau^{\prime}\right)$.

The crucial part of the study will again be the group representation $P$ of $\vartheta^{x}$ on $\operatorname{Hom}\left(U, U^{*}\right)$ defined by

$$
P(\delta) X=\rho^{*}(\delta) X \rho(\delta) \quad \delta \in \vartheta^{x}, \quad X \in \operatorname{Hom}\left(U, U^{*}\right) .
$$

It is this group representation $P$ which will be our concern for the rest of this section.

Consider $\vartheta$ as an algebra over $\mathfrak{f}_{0}$. Let $r$ and $l$ denote the right-regular representation of $\vartheta^{0}$ and the left-regular representation of $\vartheta$ over $\mathfrak{f}_{0}$, respectively, and $R$ and $L$ the associated group representations of $\vartheta^{x}$ and $\vartheta^{0^{x}}$ on $\operatorname{Hom}\left(\vartheta, \vartheta^{*}\right)$. We will construct a $T \in \operatorname{Sym}(\vartheta)$ which will play the same role in structuring the representations $R$ and $L$ as the $T$ considered in Lemma 4.1. Consider an $X \in \operatorname{Hom}\left(\vartheta, \vartheta^{*}\right)$. We define the conditions (r), (l) and (g) for such an $X$ as follows.

(r) $r^{*}(\delta) X=X r(\tau(\delta)), \delta \in \vartheta$

(l) $l^{*}(\delta) X=X l(\tau(\delta)), \delta \in \vartheta$,

(g) $\tau^{*} X \tau=X$.

The $T \in \operatorname{Sym}(\vartheta)$ will satisfy all three of these conditions and will be uniquely determined by them. We will observe below that any two conditions (r), (g) or (l), (g) determines the other (l) or (r) respectively.

Let $T_{0}: K \times K \rightarrow k_{0}$ be defined by

$$
T_{0}(\delta, \gamma)=\operatorname{tr}_{K / \mathrm{t}_{0}}(\delta \tau(\gamma)), \quad \delta, \gamma \in K .
$$

Since $\operatorname{tr}_{K / t_{0}}(g(\delta))=\operatorname{tr}_{K / t_{0}}(\delta)$ for all $\delta \in K$ and $g \in G, G$ the Galois group of $K$ over $\mathfrak{f}_{0}$, and since $\tau \in G$ it follows that

$$
\begin{aligned}
T_{0}(\delta, \gamma) & =\operatorname{tr}_{K / \mathrm{t}_{0}}(\delta \tau(\gamma))=\operatorname{tr}_{K / \mathrm{t}_{0}}(\tau(\delta \tau(\gamma))) \\
& =\operatorname{tr}_{K / \mathrm{t}_{0}}(\gamma \tau(\delta))=T_{0}(\gamma, \delta)
\end{aligned}
$$

and hence $T_{0} \in \operatorname{Sym}(\mathbf{K}), K$ viewed as a vector space over $\mathfrak{f}_{0}$. Essentially, the same argument shows that

$$
T_{0}(g(\delta), g(\gamma))=T_{0}(\delta, \gamma), \quad g \in G, \delta, \gamma \in K,
$$

using the commutativity of $G$. Take now $\delta, \gamma, \alpha \in K$. Then

$$
\begin{aligned}
T_{0}(\delta \alpha, \gamma) & =\operatorname{tr}_{K / t_{0}}(\delta \alpha \tau(\gamma))=\operatorname{tr}_{K / t_{0}}(\tau(\delta \alpha \tau(\gamma)))=\operatorname{tr}_{K / t_{0}}(\gamma \tau(\alpha) \tau(\delta)) \\
& =T_{0}(\gamma \tau(\alpha), \delta)=T_{0}(\delta, \gamma \tau(\alpha)) .
\end{aligned}
$$

We summarize the properties of $T_{0}$ in the next lemma. 
LEMma 7.1. Let $T_{0}: K \times K \rightarrow \mathfrak{f}_{0}$ be defined as above. Then $T_{0}=T_{0}^{*}$ and (i) $g^{*} T_{0} g=T_{0}, g \in G$,

(ii) $r^{*}(\delta) T_{0}=T_{0} r(\tau(\delta)), \delta \in K$,

(iii) $l^{*}(\delta) T_{0}=T_{0} l(\tau(\delta)), \delta \in K$.

Condition (iii) follows automatically from conditions (i) and (ii) by noting

$$
\begin{aligned}
T_{0}(\delta \alpha, \beta) & =T_{0}(\tau(\delta \alpha), \tau(\beta))=T_{0}(\tau(\alpha) \tau(\delta), \tau(\beta)) \quad \text { by (i) } \\
& =T_{0}(\tau(\alpha), \tau(\beta) \delta) \quad \text { by (ii) } \\
& =T_{0}(\tau(\alpha), \tau(\tau(\delta) \beta)) \quad \text { by (i) } \\
& =T_{0}(\alpha, \tau(\delta) \beta) \quad \text { by (i). }
\end{aligned}
$$

We will use $T_{0}$ to build $T$. The required conditions for $T$ force the following definitions. For $0<\mu<s$ define

$$
T_{\mu}: K j^{\mu} \times K j^{\mu} \rightarrow \mathfrak{I}_{0}
$$

by

$$
T_{\mu}\left(\gamma j^{\mu}, \delta j^{\mu}\right)=T_{0}\left(\gamma, \delta j^{\mu} \tau\left(j^{\mu}\right)\right), \quad \gamma, \delta \in K
$$

Put

$$
T=\oplus \sum_{0<\mu<s} T_{\mu}
$$

From $\tau(j) j=a \in K_{0}$ it follows that $j \tau(j)=j a j^{-1}$. For any $\alpha \in K$ we will denote the conjugates of $\alpha$ over $\mathfrak{t}$ by $\alpha^{(0)}, \ldots, \alpha^{(s-1)}$, where $\alpha=\alpha^{(0)}$. Hence $j \tau(j)=a^{(1)}$ by condition (i). It follows that $j^{\mu} \sigma\left(j^{\mu}\right)=a^{(1)} \ldots a^{(\mu)} \in K_{0}$ since $\tau\left(j a j^{-1}\right)=j(\tau(a)) j^{-1}=j a j^{-1}$ by the abelian property of the Galois group of $K$ over $\mathfrak{f}_{0}$. Thus,

$$
T_{\mu}\left(\gamma j^{\mu}, \delta j^{\mu}\right)=\operatorname{tr}_{K / \mathrm{f}_{0}}\left(a^{(1)} \ldots a^{(\mu)} \gamma \tau(\delta)\right), \quad \gamma, \delta \in K .
$$

Theorem. 7.1. Let $T$ be defined as above. Then $T=T^{*}$. Further, for $\gamma, \delta \in \vartheta$

(i) $T\left(j \gamma j^{-1}, j \delta j^{-1}\right)=T\left(a \gamma a^{-1}, \delta\right)$,

(ii) $T(\tau(\gamma), \tau(\delta)),=T(\gamma, \delta)$. Hence condition $(g)$.

(iii) $T(\gamma \alpha, \delta)=T(\gamma, \delta \tau(\alpha)), \alpha \in \vartheta$. Hence condition $(r)$.

(iv) $T(\alpha \gamma, \delta)=T(\gamma, \tau(\alpha), \delta), \alpha \in \vartheta$. Hence condition (l).

Proof. As above conditions (ii) and (iii) imply condition (iv). Also $T=T^{*}$ is obvious.

We will begin by proving (i). Let $a_{[\mu]}=a^{(1)} \ldots a^{(\mu)}$. Consider $\gamma=\sum x_{\mu} j^{\mu}$ and $\delta=\Sigma y_{\mu} j^{\mu} \in \vartheta, x_{\mu}, y_{\mu} \in K$. Then

$$
j \gamma j^{-1}=\sum x_{\mu}(1) j^{\mu}, \quad a \gamma a^{-1}=\sum a \cdot a^{(\mu)-1} x_{\mu} j^{\mu} .
$$

By definition

$$
T\left(j \gamma j^{-1}, j \delta j^{-1}\right)=\sum_{\mu} T_{\mu}\left(x_{\mu}^{(1)} j^{\mu}, y_{\mu}^{(1)} j^{\mu}\right)=\sum_{\mu} \operatorname{tr}_{K / \mathrm{z}_{0}}\left(a_{[\mu]} x_{\mu}^{(1)} \tau\left(y_{\mu}^{(1)}\right)\right) .
$$


Since $\operatorname{tr}_{K / t_{0}}$ is invariant relative to the action of $G$ and

$$
j^{-1} a_{[\mu]} j=a_{[\mu]} a a^{(\mu)^{-1}} .
$$

It follows that the sum on the right becomes

$$
\sum_{\mu} \operatorname{tr}_{K / t_{0}}\left(a_{[\mu]} a a^{(\mu)^{-1}} x_{\mu} \tau\left(y_{\mu}\right)\right)=T\left(a \gamma a^{-1}, \delta\right) .
$$

The proof of (ii) goes as follows. Let $\tau(\gamma)=\Sigma x_{\mu}^{\prime} j^{\mu}$. Then

$$
x_{s-\mu}^{\prime}=\frac{1}{b}\left(a_{[\mu]} \tau\left(x_{\mu}\right)\right)^{(s-\mu)} .
$$

Consider

$$
T(\tau(\gamma), \tau(\delta))=\sum_{\mu} \operatorname{tr}_{K / \mathrm{t}_{0}}\left(a_{[\mu]} x_{\mu}^{\prime} \tau\left(y_{\mu}^{\prime}\right)\right)=\sum_{\mu} \operatorname{tr}_{K / \mathrm{f}_{0}}\left(a_{[s-\mu]} x_{s-\mu}^{\prime} \tau\left(y_{s-\mu}^{\prime}\right)\right) .
$$

Using Galois invariance again along with the formula for $x_{s-\mu}^{\prime}$, we can easily see that the last sum can be written

$$
\frac{1}{b \tau(b)} \sum_{\mu} \operatorname{tr}_{K / \mathrm{t}_{0}}\left(a_{[s-\mu]}^{(\mu)} a_{[\mu]}^{2} \tau\left(x_{\mu}\right) y_{\mu}\right) .
$$

However, $N_{K_{0} / t_{0}}(a)=N_{K / \mathrm{t}}(a)=b \tau(b)$ and $N_{K_{0} / \mathbb{t}_{0}}(a)=a_{[\mu]} a_{[a-\mu]}^{(\mu)}$ which implies that this sum is

$$
\sum_{\mu} \operatorname{tr}_{K / \mathbb{f}_{0}}\left(a_{[\mu]} \tau\left(x_{\mu}\right) y_{\mu}\right)=T(\delta, \gamma)=T(\gamma, \delta) .
$$

To prove (iii) it is sufficient to show that for $x, y, z \in K$ and $0 \leqslant \mu, \nu, \omega<$ $s$ we have

$$
T\left(x j^{\nu} z j^{\omega}, y j^{\mu}\right)=T\left(x j^{\nu}, y j^{\mu} \tau\left(z j^{\omega}\right)\right) .
$$

If $\nu+\omega \neq \mu$ and $\nu+\omega \neq \mu+s$ both sides are 0 . We will therefore assume that $\nu+\omega=\mu$ or $\nu+\omega=\mu+s$. The proof in either case is the same so we will take $\nu+\omega+\mu$. By definition

$$
T\left(x j^{\nu} z j^{\omega}, y j^{\mu}\right)=\operatorname{tr}_{K / \mathrm{f}_{0}}\left(a_{[\mu]} x z^{(\nu)} \tau(y)\right)
$$

and

$$
\begin{aligned}
T\left(x j^{\nu}, y^{\mu} \tau\left(z j^{\omega}\right)\right) & =T\left(x j^{\nu}, y a_{[\omega]}^{(\nu)} \tau\left(z^{(\nu)}\right) j^{\nu}\right) \\
& =\operatorname{tr}_{K / z_{0}}\left(a_{[\nu]} a_{[\omega}^{(\nu)} y \tau\left(z^{(\nu)}\right) \tau(x)\right) \\
& =\operatorname{tr}_{K / z_{0}}\left(a_{[\mu]} x z^{(\nu)} \tau(y)\right)=T\left(x j^{\nu} z j^{\omega}, y j^{\mu}\right)
\end{aligned}
$$

which is what we wanted to prove.

Thus, we have found a $T \in \operatorname{Sym}(\vartheta)$ satisfying conditions (r), (l) and (g). Below, we will show $T$ is uniquely determined by these conditions.

Corollary. $T \cdot \tau \in \operatorname{Sym}(\vartheta)$ and satisfies

$$
\begin{aligned}
& r^{*}(\delta)(T \cdot \tau)=(T \cdot \tau) l(\delta), \quad \delta \in \vartheta, \\
& l^{*}(\delta)(T \cdot \tau)=(T \cdot \tau) r(\delta), \quad \delta \in \vartheta .
\end{aligned}
$$


Proof. Simply observe that $\tau^{2}=\tau$ and from (iii) of the preceding theorem

$$
(T \cdot \tau)^{*}=\tau^{*} T=T \cdot \tau^{-1}=T \cdot \tau
$$

and $T \cdot \tau$ is symmetric. The remaining assertions are equally obvious.

We call any $X \in \operatorname{Hom}\left(\boldsymbol{\vartheta}, \vartheta^{*}\right)$ satisfying the condition (r) an $r$-form and the condition (1) an $l$-form. The set of all symmetric $r$-forms will be denoted by $\delta r$ and the set of all symmetric $l$-forms by $\delta l$. Before describing the sets $\delta r$ and $\delta l$ we observe that $T$ vanishes off the 'diagonal' $K j^{\mu} \times K j^{\mu}, 0 \leqslant \mu<s$ and that a symmetric $r$-form or a symmetric $l$-form is uniquely determined by its 'top row' $K \times K j^{\mu}, 0 \leqslant \mu<s$.

LEMMA 7.1. Let $\vartheta_{0}$ be the space of all $\delta \in \vartheta$ satisfying $\tau(z)=z$. Then

(1) $\delta l=\operatorname{Tr}\left(\vartheta_{0}\right)$,

(2) $\delta r=T l\left(\vartheta_{0}\right)$.

(3) In particular, $\mathfrak{1}_{0} T=\delta r \cap \delta l$ and $T$, up to constant $\mathfrak{f}_{0}$-multiple, is the unique $r$ and l-form that is symmetric.

Proof. We will prove (1). Clearly $\operatorname{Tr}\left(\vartheta_{0}\right) \subset \mathcal{\delta}_{l}$. Consider an $X \in \delta l$ and the linear mapping of $K$ over $\mathfrak{f}_{0}$ given by

$$
x \rightarrow X\left(x, j^{\mu}\right), \quad x \in K .
$$

There exists an $\eta_{\mu} \in K$ such that $X\left(x, j^{\mu}\right)=\operatorname{tr}_{K / t_{0}}\left(\eta_{\mu} x\right), x \in K$. Hence, by the definition of $T$ on $K \times K$ it follows that

$$
X\left(x, y j^{\mu}\right)=X\left(\tau(y) x, j^{\mu}\right)=\operatorname{tr}_{K / t_{0}}\left(\eta_{\mu} x \tau(y)\right)=T\left(x, y \tau\left(\eta_{\mu}\right)\right)
$$

for all $x, y \in K$. Thus,

$$
X=r^{*}\left(j^{-\mu} \tau\left(\eta_{\mu}\right)\right) T
$$

on $K \times K j^{\mu}, 0 \leqslant \mu<s$. Consider $\eta=\Sigma_{0<\mu<s} j^{-\mu} \tau\left(\eta_{\mu}\right)$. By condition (l) $\tau(\eta)=\eta$ and $r^{*}(\eta) T$ is symmetric. Indeed, $r^{*}(\eta) T$ is a symmetric $l$-form. Since $T$ vanishes off the diagonal $X=r^{*}(\eta) T$ on $K \times K j^{\mu}$, for all $0 \leqslant \mu<s$. Thus, since both sides are symmetric $l$-forms $X=r^{*}(\eta) T$ and $X \in \operatorname{Tr}\left(\vartheta_{0}\right)$.

Property (2) is proved in a similar fashion. We will proceed to property (3). For an $X \in \operatorname{Sym}(\vartheta)$ which is both an $l$-form and an $r$-form we can write $X=\operatorname{Tr}(\eta)=T l\left(\eta^{\prime}\right), \eta, \eta^{\prime} \in \vartheta_{0}$. Since $T$ is invertible $r(\eta)=l\left(\eta^{\prime}\right)$ which immediately implies $\eta=\eta^{\prime} \in \mathfrak{f}_{0}$. Hence our lemma has been proved.

Consider the representation of $\vartheta^{x}$ on $\vartheta$ defined by setting

$$
\varepsilon(\gamma) \delta=\tau(\gamma) \delta \gamma, \quad \gamma \in \vartheta^{*}, \quad \delta \in \vartheta .
$$

Let $\vartheta_{0} \subset \vartheta$ be defined as in the preceding lemma. Then $c \vartheta_{0}$ is the eigenvalue $(-1)$-space of $\tau$ and $\vartheta=\vartheta_{0} \oplus c \vartheta_{0}$. Clearly, $\vartheta_{0}$ and $c \vartheta_{0}$ are $\varepsilon$-invariant. We will need that they are $\varepsilon$-irreducible as well. The proof runs as follows. Let $U$ be an $\varepsilon$-invariant and irreducible subspace of $\vartheta_{0}$ and $0 \neq z_{0} \in U$. From

$$
\varepsilon\left(z_{0}^{\mu}\right) z_{0}=z_{0}^{2 \mu+1} \in U \text { and } \varepsilon\left(1+z_{0}^{\mu}\right) z_{0}=z_{0}^{2 \mu+1}+2 z_{0}^{\mu+1}+z_{0} \in U
$$

it follows that all powers of $z_{0}$ are in $U$. In particular $1 \in U$. Let $1, \theta, \theta^{s-1}$ be a basis of $K_{0}$ over $\mathfrak{f}_{0}$. From

$$
\varepsilon\left(\theta^{\mu}\right) \cdot 1 \varepsilon U \text { and } \varepsilon\left(1+\theta^{\mu}\right) \cdot 1 \in U
$$


it follows that all powers of $\theta$ are in $U$ and hence $K_{0} \subset U$. Finally the elements $\theta^{\nu} j^{\mu}+\tau\left(\theta^{\nu} j^{\mu}\right)$ are in $U$ since $\varepsilon\left(j^{\mu}\right) \theta^{\nu}$ and $\varepsilon\left(1+j^{\mu}\right) \theta^{\nu}$ are in $U$, $\mu, \nu \in Z$. However, these elements $\operatorname{span} \vartheta_{0}$ over $\mathfrak{f}_{0}$ as $\mu, \nu$ run over the integers implying $U=\vartheta_{0}$.

We are now ready to study the group representations $R$ and $L$ using the elements $T$ and $T \cdot \tau$ as our main tools. Consider $\operatorname{End}_{\mathrm{f}}(\vartheta)$ as a subalgebra of $\operatorname{End}(\vartheta)$. Its dimension over $\mathfrak{f}_{0}$ is $2 s^{4}$. Define

$$
W=T \operatorname{End}_{\mathfrak{f}} \vartheta \subset \operatorname{Hom}\left(\boldsymbol{\vartheta}, \vartheta^{*}\right), \quad V=T \cdot \tau \operatorname{End}_{\mathfrak{f}} \vartheta \subset \operatorname{Hom}\left(\boldsymbol{\vartheta}, \vartheta^{*}\right) ;
$$

clearly, both are $\mathfrak{f}_{0}$-subspaces of dimension $2 s^{4}$ over $\mathfrak{f}_{0}$. We will show that $W$ is the eigenvalue $\left(-c^{2}\right)$ subspace and $V$ is the eigenvalue $c^{2}$ subspace relative to the endomorphism $R(c)$. Once this has been shown we have $W \cap V=(0)$ and arguing by dimension

$$
\operatorname{Hom}\left(\vartheta, \vartheta^{*}\right)=W \oplus V
$$

Take $X=T Y \in W$ where $Y \in \operatorname{End}_{\mathfrak{f}}(\vartheta)$. Then

$$
R(c) X=r^{*}(c) T Y r(c)=\operatorname{Tr}(-c) \operatorname{Yr}(c)=-c^{2} T Y=-c^{2} X
$$

since $Y$ is linear over $\mathfrak{l}^{2} \in \mathfrak{f}_{0}$. Similarly, for $X \in V$ we have $R(c) X=$ $c^{2} X$ and our assertion is proved.

The theory of central simple algebras gives

$$
\operatorname{End}_{\mathfrak{f}}(\vartheta)=l(\vartheta) \otimes_{\mathfrak{t}} r\left(\vartheta^{0}\right) \cong \vartheta \otimes_{\mathfrak{t}} \vartheta^{0}
$$

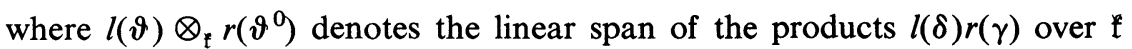
where $\delta, \gamma \in \vartheta$. We will use this strongly in our analysis of the spaces $W$ and $V$.

Consider $W=T \operatorname{End}_{\mathrm{f}}(\boldsymbol{\vartheta})$. We begin by showing that $W$ is $R$ and $L$ invariant. Take $X=T l(\delta) r(\gamma) \in W$ and $\alpha \in \vartheta^{x}$. Then

$$
R(\alpha) X=r^{*}(\alpha) T l(\delta) r(\gamma) r(\alpha)=\operatorname{Tl}(\delta) r(\alpha \gamma \tau(\alpha)) \in W
$$

and hence $W$ is $R$-invariant. A similar argument shows that $W$ is $L$-invariant as well. Observe that for $\delta, \gamma \in \vartheta_{0}$ we have $\alpha \gamma \tau(\alpha)$ and $\alpha \delta \tau(\alpha)$ in $\vartheta_{0}$. Thus far $\delta, \gamma \in \vartheta_{0}$ and $X=T l(\delta) r(\gamma)$ a refinement of the above argument shows that $R(\alpha) X$ and $L(\alpha) X$ are in $\operatorname{Tl}\left(\vartheta_{0}\right) \bigotimes_{\mathfrak{f}} r\left(\vartheta_{0}\right)$. It follows that the subspaces

$$
W_{1}=T l\left(\vartheta_{0}\right) \otimes_{\mathfrak{f}} r\left(\vartheta_{0}\right), \quad W_{2}=T l\left(\vartheta_{0}\right) \otimes_{\mathfrak{f}} r\left(\vartheta_{0}\right) r(c)=W_{1} r(c)
$$

are $R$ and $L$-invariant. There subspaces $W_{1}$ and $W_{2}$ are easily seen to be the symmetric and alternating elements, respectively, of $W$ and $W=W_{1} \oplus W_{2}$. These subspaces are also irreducible with respect to the joint action of $R$ and $L$. This need only be proved for $W_{1}$. Identifying $W_{1}$ with $\vartheta_{0} \otimes_{\mathfrak{f}} \vartheta_{0}$ as vector spaces over $\mathfrak{f}$ the representations $R$ and $L$ on $W_{1}$ induce representations $R_{1}$ and $L_{1}$, respectively, on $\vartheta_{0} \otimes_{\mathfrak{f}} \vartheta_{0}$ given by

$$
\begin{aligned}
& R_{1}(\delta)(\alpha \otimes \beta)=(\tau(\delta) \alpha \delta) \otimes \beta, \\
& L_{1}(\delta)(\alpha \otimes \beta)=\alpha \otimes(\tau(\delta) \beta \delta), \quad \alpha, \beta \in \vartheta_{0} .
\end{aligned}
$$

Now as we have seen the mappings $\alpha \rightarrow \tau(\delta) \alpha \delta$ of $\vartheta_{0}$ as $\delta$ runs over $\vartheta^{x}$ act irreducibly. The joint action of $R_{1}$ and $L_{1}$ on $\vartheta_{0} \otimes_{t} \vartheta_{0}$ is the same as the tensor product of this irreducible action with itself and hence is irreducible. 
Thus, $W_{1}$ and also $W_{2}$ are irreducible with respect to the joint action of $R$ and $L$.

We shall now decompose $W_{1}$ and hence $W_{2}$ into $R$-invariant and irreducible subspaces. Before we considered $\delta r$ the space of symmetric $r$-forms with respect to $\tau$ and found that $\delta r=T l\left(\vartheta_{0}\right)$. To indicate the dependence of $\delta r$ upon $\tau$ we shall now write $\delta r=\delta r(\tau)$. It is obvious that the space $\mathfrak{A} r=$ $\mathfrak{U} r(\tau)=\mathcal{S} r(\tau) r(c)$ consists of all the alternating $r$-forms with respect to $\tau$ and is in fact the space $\mathscr{Q}(\vartheta, r, \tau)$ defined above under the identification $\operatorname{Pol}(\vartheta)$ with $\operatorname{Alt}(\vartheta)$.

The space $\mathcal{S}(\tau)$ is obviously an $L$-invariant subspace of $W_{1}$ the action on a typical element $X=T l(\delta), \delta \in \vartheta_{0}$ being given by

$$
L(\gamma) X=l^{*}(\gamma) T l(\delta) l(\gamma)=T l(\tau(\gamma) \delta \gamma) \in \delta r .
$$

Since the mappings $\delta \rightarrow \tau(\gamma) \delta \gamma$ of $\vartheta_{0}$ act irreducibly as $\gamma$ runs over $\vartheta^{x}$, it follows that $\delta r(\tau)=T l\left(\vartheta_{0}\right)$ is $L$-invariant and irreducible. Observe that the dimension of $\operatorname{S} r(\tau)$ over $\mathfrak{f}_{0}$ is $s^{2}$. Now let $1=\alpha_{0}, \alpha_{1}, \ldots, \alpha_{t}, t=s^{2}-1$ be a basis of $\vartheta_{0}$ over $\mathfrak{f}_{0}$ and consider the spaces

$$
\delta r(\tau) r\left(\alpha_{j}\right)=T l\left(\vartheta_{0}\right) r\left(\alpha_{j}\right), \quad 0 \leqslant j \leqslant t .
$$

For $X \in T l\left(\vartheta_{0}\right) r\left(\alpha_{j}\right)$, we have $L(\delta) X$ in $T l\left(\vartheta_{0}\right) r\left(\alpha_{j}\right)$ and it is easy to see that $T l\left(\vartheta_{0}\right) r\left(\alpha_{j}\right)$ is an $L$-invariant and irreducible subspace consisting of all symmetric $r$-forms corresponding to the (not necessarily positive) involution $\tau^{\prime}(\delta)=\alpha_{j} \tau(\delta) \alpha_{j}^{-1}$. Arguing by dimension we have

$$
W_{1}=\oplus \sum_{j=0}^{t} \delta r(\tau) r\left(\alpha_{j}\right)
$$

Analogous results for $W_{2}=W_{1} r(c)$ immediately follow. We summarize these results as follows.

LEMMA 7.2. Let $W=T \operatorname{End}_{\mathfrak{f}}(\vartheta)=T l(\vartheta) \otimes_{\mathfrak{f}} r\left(\vartheta^{\vartheta}\right)$ and set

$$
W_{1}=\operatorname{Tl}\left(\vartheta_{0}\right) \otimes_{\mathfrak{t}}\left(\vartheta_{0}\right), \quad W_{2}=W_{1} r(c) \text {. }
$$

Then $W=W_{1} \oplus W_{2}$ is the decomposition of $W$ into the direct sum of its symmetric elements $W_{1}$ and its alternating elements $W_{2}$.

(i) The space $\delta r(\tau)=T l\left(\vartheta_{0}\right)$ of symmetric $r$-forms relative to $\tau$ is an $L$-invariant and irreducible subspace and

$$
W_{1}=\bigoplus \sum_{j=0}^{t} \delta r(\tau) r\left(\alpha_{j}\right)
$$

where $i=\alpha_{0}, \ldots, \alpha_{t}, t=s^{2}-1$ is a basis of $\vartheta_{0}$ over $\mathfrak{f}_{0}$.

(ii) The space $\mathfrak{A} r(\tau)=\delta r(\tau) r(c) \cong \mathscr{Q}(\vartheta, r, \tau)$ of alternating $r$-forms relative to $\tau$ is an $L$-invariant and irreducible subspace and

$$
W_{2}=W_{1} r(c)=\bigoplus \sum_{j=0}^{t} \delta r(\tau) r(c) r\left(\alpha_{j}\right)=\bigoplus \sum_{j=0}^{t} \mathfrak{A}_{r}(\tau) r\left(\alpha_{j}\right)
$$

with notation as in (1).

Moreover, $W_{1}$ and $W_{2}$ are irreducible with respect to the joint action of $L$ 
and $R$. There is a completely analogous set of results decomposing $W_{1}$ and $W_{2}$ into $R$-invariant and irreducible spaces.

Consider $V=T \cdot \tau \cdot \operatorname{End}_{\mathrm{f}}(\boldsymbol{\vartheta})=T \cdot \tau \cdot l(\vartheta) \otimes r\left(\boldsymbol{\vartheta}^{\boldsymbol{\vartheta}}\right)$. We may identify $V$ with $\vartheta \otimes_{\mathfrak{f}} \vartheta^{0}$ and observe that $V$ is $R$ and $L$-invariant where this identification associates to $R$ and $L$ the representation $R_{2}$ and $L_{2}$ on $\vartheta \otimes_{\mathfrak{f}} \vartheta^{0}$ given as follows. If $\delta, \alpha, \beta \in \vartheta$ then

$$
R_{2}(\delta)(\alpha \otimes \beta)=(\alpha \delta) \otimes(\beta \delta), \quad L_{2}(\delta)(\alpha \otimes \beta)=(\delta \alpha) \otimes(\delta \beta) .
$$

Let $V_{1}^{\prime}$ be the space of symmetric tensors in $\vartheta \otimes_{f} \vartheta^{0}$ and $V_{2}^{\prime}$ the space of alternating tensors in $\vartheta \otimes_{\mathfrak{t}} \vartheta^{0}$. It is obvious that both $V_{1}^{\prime}$ and $V_{2}^{\prime}$ are invariant with respect to $R_{2}$ and $L_{2}$ jointly. Moreover, if $V_{1}$ and $V_{2}$ are the corresponding subspaces of $V$ then $V=V_{1} \oplus V_{2}$ where $V_{1}$ and $V_{2}$ are $R$ and $L$-invariant. Also, as is known, the spaces $V_{1}^{\prime}$ and $V_{2}^{\prime}$ are irreducible with respect to the mappings $\alpha \otimes \beta \rightarrow X \alpha \otimes X \beta, X \in G L_{2}(\vartheta)$. Finally since $V_{1} \subset$ $\operatorname{Sym}(\vartheta)$ and $V_{2} \subset \operatorname{Alt}(\vartheta)$ we have $\operatorname{Sym}(\vartheta)=W_{1} \oplus V_{1}$ and $\operatorname{Alt}(\vartheta)=W_{2} \otimes$ $V_{2}$.

Let $\rho$ be an arbitrary representation of the algebra $\vartheta^{0}$ over $\mathfrak{f}_{0}$ on a vector space $U$ over $\mathfrak{f}_{0}$ and $P$ the induced group representation of $\vartheta^{x}$ on $\operatorname{Hom}\left(U, U^{*}\right)$. The theory of simple algebras allows us to identify $\rho$ with $q \cdot r$, $r$ the regular representation of $\vartheta^{0}$ over $\mathfrak{l}_{0}$ and $q \geqslant 1$ an integer, up to $\mathfrak{f}_{0}$-equivalence. Hence, without loss of generality, we assume $\rho=q \cdot r$ and

$$
U=\bigoplus \sum_{\mu=1}^{q} \vartheta_{\mu}
$$

where each $\vartheta_{\mu}=\vartheta$ is $\rho$-invariant and the restriction of $\rho(\delta)$ to $\vartheta_{\mu}$ is $r$. Hence, $U^{*}=\bigoplus \sum_{\mu=1}^{q} \vartheta_{\mu}^{*}$ and

$$
\operatorname{Hom}\left(U, U^{*}\right)=\bigoplus \sum_{\mu, \nu=1}^{q} \operatorname{Hom}\left(\vartheta_{\mu}, \vartheta_{\nu}^{*}\right)
$$

where each of the factors $\operatorname{Hom}\left(\vartheta_{\mu}, \vartheta_{\nu}^{*}\right)=\operatorname{Hom}\left(\vartheta, \vartheta^{*}\right)$ is $P$-invariant and the restriction of $P(\delta)$ to $\operatorname{Hom}\left(\vartheta_{\mu}, \vartheta_{\nu}^{*}\right)$ is $R(\delta)$. Thus, the study of the group representation $P$ reduces to Lemma 7.2 and the discussion immediately following it.

$\operatorname{Consider} \operatorname{Hom}\left(\boldsymbol{\vartheta}_{\mu}, \boldsymbol{\vartheta}_{\boldsymbol{\nu}}^{*}\right)=\operatorname{Hom}\left(\boldsymbol{\vartheta}, \boldsymbol{\vartheta}^{*}\right)$ and write

$$
\begin{array}{rll}
\operatorname{Sym}\left(\vartheta_{\mu}\right)=W_{1}(\mu) \oplus V_{1}(\mu), & W_{1}(\mu)=W_{1}, & V_{1}(\mu)=V_{1}, \\
\operatorname{Alt}\left(\vartheta_{\mu}\right)=W_{2}(\mu) \oplus V_{2}(\mu), & W_{2}(\mu)=W_{2}, & V_{2}(\mu)=V_{2} .
\end{array}
$$

For $\mu \neq \nu$ let

$\operatorname{Sym}(\mu, \nu)=$ the symmetric elements in $\operatorname{Hom}\left(\vartheta_{\mu}, \vartheta_{\nu}^{*}\right) \oplus \operatorname{Hom}\left(\vartheta_{\nu}, \vartheta_{\mu}^{*}\right)$,

$$
\operatorname{Alt}(\mu, \nu)=\text { the alternating elements in } \operatorname{Hom}\left(\boldsymbol{\vartheta}_{\mu}, \boldsymbol{\vartheta}_{\nu}^{*}\right) \oplus \operatorname{Hom}\left(\boldsymbol{\vartheta}_{\nu}, \boldsymbol{\vartheta}_{\mu}^{*}\right) \text {. }
$$

The mapping $X \rightarrow X \oplus X^{*}$ of $\operatorname{Hom}\left(\boldsymbol{\vartheta}_{\mu}, \boldsymbol{\vartheta}_{\nu}^{*}\right)$ into $\operatorname{Hom}\left(\boldsymbol{\vartheta}_{\mu}, \boldsymbol{\vartheta}_{\nu}^{*}\right) \oplus \operatorname{Hom}\left(\boldsymbol{\vartheta}_{\nu}, \boldsymbol{\vartheta}_{\mu}^{*}\right)$ determines an isomorphism

$$
\operatorname{Hom}\left(\vartheta_{\mu}, \vartheta_{\nu}^{*}\right) \cong \operatorname{Sym}(\mu, \nu)
$$


while the mapping $X \rightarrow X \oplus\left(-X^{*}\right)$ of $\operatorname{Hom}\left(\boldsymbol{\vartheta}_{\mu}, \vartheta_{\nu}^{*}\right)$ into $\operatorname{Hom}\left(\boldsymbol{\vartheta}_{\mu}, \vartheta_{\nu}^{*}\right) \oplus$ $\operatorname{Hom}\left(\vartheta_{\nu}, \vartheta_{\mu}^{*}\right)$ determines an isomorphism

$$
\operatorname{Hom}\left(\vartheta_{\mu}, \vartheta_{\nu}^{*}\right) \simeq \operatorname{Alt}(\mu, \nu) \text {. }
$$

In the following these isomorphisms will be understood and we may write

$$
\operatorname{Sym}(\mu, \nu)=W_{s}(\mu, \nu) \oplus V_{s}(\mu, \nu)
$$

where $W \simeq W_{s}(\mu, \nu)$ and $V \simeq V_{s}(\mu, \nu)$ under the first isomorphism and hence $\operatorname{Sym}(U)=W_{s}(\rho) \oplus V_{s}(\rho)$ where

$$
\begin{gathered}
W_{s}(\rho)=\oplus \sum_{\mu} W_{1}(U) \oplus \sum_{\mu<\nu} W_{s}(\mu, \nu), \\
V_{s}(\rho)=\oplus \sum_{\mu} V_{1}(\mu) \oplus \sum_{\mu<\nu} V_{s}(\mu, \nu) .
\end{gathered}
$$

Also, we may write

$$
\operatorname{Alt}(\mu, \nu)=W_{A}(\mu, \nu) \oplus V_{A}(\mu, \nu)
$$

where $W \cong W_{A}(\mu, \nu)$ and $V \cong V_{A}(\mu, \nu)$ under the second isomorphism. Then $\operatorname{Alt}(U)=W_{A}(\rho) \oplus V_{A}(\rho)$ where

$$
\begin{aligned}
W_{A}(\rho) & =\oplus \sum_{\mu} W_{2}(\mu) \oplus \sum_{\mu<\nu} W_{A}(\mu, \nu), \\
V_{A}(\rho) & =\oplus \sum_{\mu} V_{2}(\mu) \oplus \sum_{\mu<\nu} V_{A}(\mu, \nu) .
\end{aligned}
$$

Let $\delta r(\rho)=\delta r(\rho, \tau)$ be the symmetric $r$-forms on $U$ corresponding to $\rho$ and $\tau$ in the sense that $X=X^{*} \in \operatorname{Hom}\left(U, U^{*}\right)$ and

$$
\rho^{*}(\delta) X=X \rho(\tau(\delta)), \quad \delta \in \vartheta .
$$

Similarly, let $\mathfrak{A} r(\rho)=\mathfrak{A} r(\rho, \tau) \cong \mathbb{Q}(\vartheta, \rho, \tau)$ be the alternating $r$-forms on $U$ corresponding to $\rho$ and $\tau$. We have

$$
\delta r(\rho, \tau)=\oplus \sum_{\mu} W_{1}(\mu, \tau) \oplus \sum_{\mu<\nu} W_{s}(\mu, \nu, \tau)
$$

where $W_{1}(\mu, \tau) \cong T l\left(\vartheta_{0}\right)$ and $W_{s}(\mu, \nu, \tau) \simeq T l(\vartheta)$ under the first isomorphism. Also,

$$
\mathcal{Q}(\vartheta, \rho \tau) \cong \mathfrak{A} r(\rho, \tau)=\delta r(\rho, \tau) r(c) .
$$

The dimension of each of these spaces is $q^{2} s^{2}$ over $\mathfrak{f}_{0}$.

We are now in a position to discuss the original problem of this section. Let $\rho_{Q}$ be a rational representation of $\vartheta^{0}$ on the rational vector space $U=U(Q)$ and $\rho$ the corresponding representation $\vartheta^{0}$ on the vector space $U$ over $\mathfrak{f}_{0}$. Denote the set of positive involutions $\tau^{\prime}$ on $\vartheta$ coinciding with $\tau$ on the center by $[\tau]$. Recall the relation $t\left(\mathbb{Q}\left(\vartheta, \rho, \tau^{\prime}\right)\right)=\mathscr{Q}\left(\vartheta, \rho_{Q}, \tau^{\prime}\right)$ and the corresponding diagram

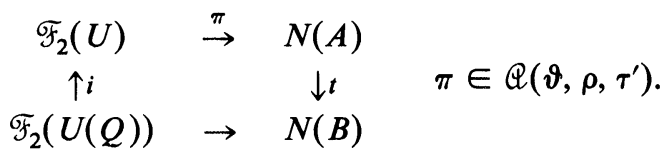


Let $\rho=r$ and take $\tau^{\prime} \in[\tau]$ where $\tau^{\prime}(\delta)=u \tau(\delta) u^{-1}, u \in \vartheta_{0}$. Consider the space $T l\left(\vartheta_{0}\right) r(u) \subset W_{1}$ and take $X=T l(\gamma) r(u), \gamma \in \vartheta_{0}$. For $\delta \in \vartheta$,

$$
r(\delta)^{*} X=r^{*}(\delta) T l(\gamma) r(u)=\operatorname{Tl}(\gamma) r(u) r\left(u \tau(\delta) u^{-1}\right)=X r\left(\tau^{\prime}(\delta)\right) .
$$

It follows that $\operatorname{Tl}\left(\vartheta_{0}\right) r(u)=\delta r\left(\tau^{\prime}\right)$, the space of all symmetric $r$-forms on $\vartheta$ corresponding to $\tau^{\prime}$. Observe that since we have already proved that $\delta r(\tau)$ is $L$-invariant and irreducible, we have $\delta r\left(\tau^{\prime}\right) L$-invariant and irreducible as well. Thus

$$
\bigcup_{\tau^{\prime} \in[\tau]} \delta r\left(\tau^{\prime}\right) \subset W_{1} .
$$

Consider the sum $\sum_{\delta \in \vartheta^{x}} R(\delta) T l\left(\vartheta_{0}\right) r(u)$. This space is both $R$ and $L$-invariant and since it is contained in $W_{1}$ which is both $R$ and $L$-invariant and irreducible under their joint action

$$
W_{1}=\sum_{\delta \in \vartheta^{x}} R(\delta) T l\left(\vartheta_{0}\right) r(u)=\sum_{\delta \in \vartheta^{x}} R(\delta) \delta r\left(\tau^{\prime}\right)
$$

and $W_{1}$ is the smallest $R$ and $L$-invariant space containing $\delta r\left(\tau^{\prime}\right)$.

Consider $\operatorname{Sr}(\tau)=T l\left(\vartheta_{0}\right)$. For any $\delta_{0} \in \vartheta_{0}$ consider $X=T l\left(\delta_{0}\right) \in \mathcal{S} r(\tau)$. Then, if $\delta \in \vartheta^{x}$

$$
R(\delta) X=r(\delta)^{*} T l\left(\delta_{0}\right) r(\delta)=T l\left(\delta_{0}\right) r(\delta \tau(\delta))
$$

where $v=\delta \tau(\delta) \in \vartheta_{0}$. Take now $\gamma \in \vartheta^{x}$ and write

$$
r(\gamma)^{*} R(\delta) X=r^{*}(\gamma) T l\left(\delta_{0}\right) r(v)=\operatorname{Tl}\left(\delta_{0}\right) r(v) r\left(v \tau(\gamma) v^{-1}\right) .
$$

Since as we saw $\tau^{\prime}(\delta)=v \tau(\gamma) v^{-1}$ is a positive involution in $[\tau]$ we have

$$
\delta r\left(\tau^{\prime}\right)=R(\delta) \delta r(\tau)
$$

where $\tau^{\prime}$ is the positive involution in $[\tau]$ given by $\tau^{\prime}(\gamma)=v \tau(\gamma) v^{-1}$ with $v=\delta \tau(\delta)$. Thus, $W_{1}$ is spanned over $\mathfrak{f}_{0}$ by $\cup_{\tau^{\prime} \in[\tau]} S_{r}\left(\tau^{\prime}\right)$. We will summarize this discussion as follows.

Lemma 7.3. Let $\tau^{\prime} \in[\tau]$ be given by $\tau^{\prime}(\delta)=u \tau(\delta) u^{-1}, u \in \vartheta_{0}$. Then

$$
\delta r\left(\tau^{\prime}\right)=T l\left(\vartheta_{0}\right) r(u) \subset W_{1} \text {. }
$$

Moreover, $W_{1}$ is the smallest $R$-invariant space containing $\delta r\left(\tau^{\prime}\right)$ and $\cup_{\tau^{\prime} \in[\tau]} \mathcal{S} r\left(\tau^{\prime}\right)$ spans $W_{1}$ over $\mathfrak{f}_{0}$.

The analogous 'alternating' result is the following.

LEMMA 7.4. Let $\tau^{\prime} \in[\tau]$ be determined by $\tau^{\prime}(\delta)=u \tau(\delta) u^{-1}, u \in \vartheta_{0}$. Then

$$
\mathcal{Q}\left(\vartheta, r, \tau^{\prime}\right) \cong T l\left(c \vartheta_{0}\right) r(u) \subset W_{2} .
$$

Moreover, $W_{2}$ is the smallest $R$-invariant space containing $\mathcal{Q}\left(\vartheta, r, \tau^{\prime}\right)$ and $\mathcal{Q}(\vartheta, r)$ spans $W_{2}$ over $\mathfrak{f}_{0}$.

In terms of commutative diagrams we can reinterpret this last lemma as follows. Considering $\operatorname{Pol}(\boldsymbol{\vartheta}) \cong \operatorname{Alt}(\boldsymbol{\vartheta})=(\boldsymbol{\vartheta} \wedge \boldsymbol{\vartheta})^{*}$ we can associate to any subspace $U$ of $\operatorname{Pol}(\vartheta) \cong \operatorname{Alt}(\vartheta)$ its dual $U^{*}$ as a subspace of $\vartheta \wedge \vartheta$ given as the set of all elements of $\vartheta \wedge \vartheta$ annihilated by $U$. Let

$$
N\left(\vartheta, r, \rho^{\prime}\right)=\mathscr{F}_{2}(\vartheta) / \mathcal{Q}(\vartheta, r, \tau)^{*}, \quad N(\vartheta, r)=\mathscr{F}_{2}(\vartheta) W_{2}^{*} .
$$


Then $\mathcal{Q}\left(\vartheta, r, \tau^{\prime}\right)$ consists of $\pi \in \mathscr{F}_{2}(\vartheta)$ which factor through to define homomorphisms of $N\left(\vartheta, r, \tau^{\prime}\right)$.

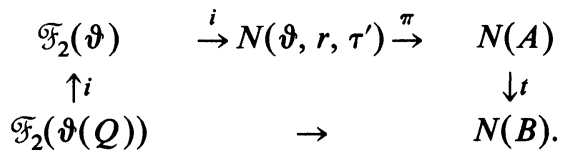

Moreover $N(\vartheta, r)$ is the 'largest' group in which every polarization $\pi \in$ $\mathbb{Q}(\vartheta, r)$ defines a homomorphism.

The generalized results can be found using Theorem 7.1 and we simply observe $\mathcal{Q}\left(\vartheta, \tau, \tau^{\prime}\right)=W_{A}(\rho, \tau) r(u)=\mathscr{Q}(\vartheta, \tau, \tau) r(u)$ where $\tau^{\prime}(\delta)=u r(\delta) u^{-1}$, $u \in \vartheta_{0}$ and state the following theorem.

THEOREM 7.2. Let $\tau^{\prime} \in[\tau]$ be given by $\tau^{\prime}(\delta)=u \tau(\delta) u^{-1}, u \in \vartheta_{0}$. Then

$$
\mathcal{Q}\left(\vartheta, \rho, \tau^{\prime}\right) \cong\left(\bigoplus \sum_{\mu} W_{1}(\mu, \tau) \oplus \sum_{\mu<\nu} W_{s}(\mu, \nu, \tau)\right) r(c) r(\mu)
$$

is a subspace of $W_{A}(\rho)$ which is the smallest $R$-invariant subspace containing $\mathbb{Q}\left(\vartheta, \rho, \tau^{\prime}\right)$. Moreover $\mathbb{Q}(\vartheta, \rho) \subset W_{A}(\rho)$ spans $W_{A}(\rho)$ as a subspace over $\mathfrak{f}_{0}$.

8. Riemann matrices. Cyclic algebras. We come now to the problem of the existence of 'Riemann Matrices'. Continue the notation of the preceding section. Thus, $\vartheta$ is a cyclic division algebra with center $\mathfrak{f}, \tau$ is a fixed positive involution for $\vartheta$ having $\mathfrak{f}_{0}$ as its fixed field in $\boldsymbol{f}^{\prime}$ and $\boldsymbol{\tau}^{\prime}$ is positive involution coinciding with $\tau$ on the center. Let $\rho_{Q}$ be a rational representation of $\vartheta^{0}$ on the rational vector space $U=U(Q)$ and $\rho$ the corresponding representation of $\vartheta^{0}$ on the vector space $U$ over $\mathfrak{f}_{0}$. Then we have the relationship

$$
t\left(\mathscr{Q}\left(\vartheta, \rho \tau^{\prime}\right)\right)=\mathscr{Q}\left(\vartheta, \rho_{Q}, \tau^{\prime}\right)
$$

given by the commutative diagram

$$
\begin{array}{ccc}
\mathscr{F}_{2}(U) & \stackrel{\pi}{\rightarrow} & N(A) \\
\uparrow i & & \downarrow t \\
\mathscr{F}_{2}(U(Q)) & \rightarrow & N(B)
\end{array} \quad \pi \in \mathbb{Q}\left(\vartheta, \rho, \tau^{\prime}\right) .
$$

Consider the real closure of this diagram,

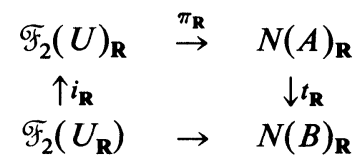

as we described in $\S \S 4$ and 6. The problem as before is to describe for each $\pi^{\prime} \in \mathbb{Q}\left(\vartheta, \rho_{Q}, \tau^{\prime}\right)$ the collection of all complex structures $J$ on $U_{\mathbf{R}}$ satisfying

$\left(\mathrm{i}^{\prime \prime}\right) \rho_{\mathbf{R}}(\alpha) J=J \rho_{\mathbf{R}}(\alpha), \alpha \in \vartheta$.

(ii") $\left(J, \pi_{\mathbf{R}}^{\prime}\right)$ is a Riemann pair.

Let $N(A)_{\mathbf{R}}=\Pi_{1}^{h^{\prime}} N_{2 p+1}\left(\mathbf{R}, \chi_{i}\right), h^{\prime}=\left[\mathfrak{f}_{0}: Q\right], 2 p=\operatorname{dim}_{\mathfrak{t}_{0}} U$ be the decomposition of $N(A)_{\mathbf{R}}$ compatible with the action of $\rho\left(\mathfrak{f}_{0}\right)$ as discussed in $\S \S 4$ and 
6. Then, $\Re\left(\vartheta, \rho_{Q}, B\right)$, the collection of all complex structures on $U_{\mathbf{R}}$ satisfying $\left(i^{\prime \prime}\right)$ and (ii") is given by

$$
\Re\left(\vartheta, \rho_{Q}, B\right)=\prod_{1}^{h^{\prime}} \oiint_{i}(A)
$$

where $\mathscr{S}_{i}(A)$ is the set of all positive definite $C R$-structures $J_{i}$ on $N_{2 p+1}\left(\mathbf{R}, \chi_{i}\right)$ such that the induced automorphism in $U_{\mathbf{R}}=N_{2 p+1}\left(\mathbf{R}, \chi_{i}\right) /$ center commutes with $\rho_{\mathbf{R}}(\vartheta)$, elementwise.

The existence of an element in $\mathscr{R}\left(\vartheta, \rho_{Q}, B\right)$ is assured as follows. Since $-c^{2}$ is totally positive in $\mathfrak{f}_{0}$ we find a totally positive square root $\sqrt{-c^{2}}$ in the algebra $\mathfrak{f}_{0} \otimes_{Q} \mathbf{R}$. The mapping of $N(A)_{\mathbf{R}}$,

$$
(\delta, \gamma) \rightarrow\left(\frac{1}{\sqrt{-c^{2}}} \rho(c) \delta, \gamma\right), \quad(\delta, \gamma) \in N(A)_{\mathbf{R}}
$$

determines such an element using the $\mathbf{R}$-linear extension of the action of $\mathfrak{f}_{0}$ on $U$ to an action of $\left(\mathfrak{f}_{0}\right)_{\mathbf{R}}=\mathfrak{I}_{0} \otimes_{Q} \mathbf{R}$ to $U_{\mathbf{R}}$.

\section{BIBLIOGRAPHY}

1. A. A. Albert, Structures of algebras, Amer. Math. Soc. Colloq. Publ., no. 24, Amer. Math. Soc. Providence, R. I., 1939.

2. L. Auslander, Lecture notes on nil-theta functions, CBMS Regional Conf. Ser. in Math., no. 34, Amer. Math. Soc., Providence, R. I., 1977.

3. N. Jacobson, Abraham Adrian Albert 1905-1972, Bull. Amer. Math. Soc. 80 (1974), 1075-1100.

4. C. L. Siegel, Lectures on Riemann matrices, Tata Institute of Fundamental Research, Bombay, 1963.

5. R. Tolimieri, Heisenberg manifolds and theta functions, Trans. Amer. Math. Soc. 239 (1978), 293-319.

6. A. Weil. Sur certaines groupes d'operateurs unitaires, Acta Math. 111 (1964), 143-211.

Department of Mathematics, Graduate School and University Center, City UniverSITY OF NEW YORK, NEW YORK 10036

Department of Mathematics, University of Connecticut, StorRs, Connecticut 06268 